\title{
Giant gamma-ray bubbles from Fermi-LAT: active galactic nucleus activity or bipolar galactic wind?
}

\section{Citation}

Su, Meng, Tracy R. Slatyer, and Douglas P. Finkbeiner. 2010. “Giant gamma-ray bubbles from Fermi-LAT: active galactic nucleus activity or bipolar galactic wind?" The Astrophysical Journal 724 (2) (November 10): 1044-1082. doi:10.1088/0004-637x/724/2/1044.

\section{Published Version}

doi:10.1088/0004-637X/724/2/1044

\section{Permanent link}

http://nrs.harvard.edu/urn-3:HUL.InstRepos:33461909

\section{Terms of Use}

This article was downloaded from Harvard University's DASH repository, and is made available under the terms and conditions applicable to Other Posted Material, as set forth at http:// nrs.harvard.edu/urn-3:HUL.InstRepos:dash.current.terms-of-use\#LAA

\section{Share Your Story}

The Harvard community has made this article openly available.

Please share how this access benefits you. Submit a story.

\section{Accessibility}




\title{
GIANT GAMMA-RAY BUBBLES FROM FERMI-LAT: ACTIVE GALACTIC NUCLEUS ACTIVITY OR BIPOLAR GALACTIC WIND?
}

\author{
Meng Su ${ }^{1}$, Tracy R. Slatyer ${ }^{1,2}$, and Douglas P. Finkbeiner ${ }^{1,2}$ \\ ${ }^{1}$ Institute for Theory and Computation, Harvard-Smithsonian Center for Astrophysics, 60 Garden Street, MS-51, Cambridge, MA 02138, USA; \\ mengsu@cfa.harvard.edu \\ 2 Physics Department, Harvard University, Cambridge, MA 02138, USA \\ Received 2010 June 2; accepted 2010 September 23; published 2010 November 10
}

\begin{abstract}
Data from the Fermi-LAT reveal two large gamma-ray bubbles, extending $50^{\circ}$ above and below the Galactic center (GC), with a width of about $40^{\circ}$ in longitude. The gamma-ray emission associated with these bubbles has a significantly harder spectrum $\left(d N / d E \sim E^{-2}\right)$ than the inverse Compton emission from electrons in the Galactic disk, or the gamma rays produced by the decay of pions from proton-interstellar medium collisions. There is no significant spatial variation in the spectrum or gamma-ray intensity within the bubbles, or between the north and south bubbles. The bubbles are spatially correlated with the hard-spectrum microwave excess known as the WMAP haze; the edges of the bubbles also line up with features in the ROSAT X-ray maps at $1.5-2 \mathrm{keV}$. We argue that these Galactic gamma-ray bubbles were most likely created by some large episode of energy injection in the GC, such as past accretion events onto the central massive black hole, or a nuclear starburst in the last $\sim 10$ Myr. Dark matter annihilation/decay seems unlikely to generate all the features of the bubbles and the associated signals in WMAP and ROSAT; the bubbles must be understood in order to use measurements of the diffuse gamma-ray emission in the inner Galaxy as a probe of dark matter physics. Study of the origin and evolution of the bubbles also has the potential to improve our understanding of recent energetic events in the inner Galaxy and the high-latitude cosmic ray population.
\end{abstract}

Key words: galaxies: active - galaxies: starburst - gamma rays: diffuse background - ISM: jets and outflows

Online-only material: color figures

\section{INTRODUCTION}

The inner Milky Way is home to a massive black hole (MBH), surrounded by clusters of young stars and giant molecular clouds (see, e.g., Morris \& Serabyn 1996, for a review). The nuclear star cluster has a half-light radius of $\sim 5 \mathrm{pc}$. Although there are indications of past activity, the black hole $(\mathrm{BH})$ is quiescent today.

$\mathrm{Fe} \mathrm{K} \alpha$ echoes from molecular clouds around Sgr A* have been understood as relics of activity in the past few hundred years (Sunyaev et al. 1993; Koyama et al. 1996). On a longer timescale, one might expect relics of past activity in high-energy CRs and hot gas, perhaps far off the disk. The most obvious observables would be $e^{-} \mathrm{CR}$ (visible in inverse Compton (IC) gammas and microwave synchrotron) and thermal emission (Xrays).

This work presents a multiwavelength study of the inner Galaxy and identifies several large-scale (tens of degrees) gamma-ray features, most notably two large (spanning $-50^{\circ}<$ $b<50^{\circ}$ ) structures that we refer to as the "Fermi bubbles." We suggest that these bubbles are associated with previously discovered structures in the X-rays and microwaves, and possibly with analogous smaller-scale structures visible in the FIR.

\subsection{Previous High-energy Excesses}

Observations of gamma-ray emission in the inner Galaxy at $E \lesssim 1 \mathrm{GeV}$ go back decades to $C O S-B$ (Strong 1984; Strong et al. 1987) and SAS-2 (Fichtel et al. 1975; Kniffen \& Fichtel 1981; see Bloemen 1989, for a review). Later data from the EGRET experiment aboard the Compton Gamma-ray Observatory extended to the high-energy side of the $\pi^{0}$ bump (Smialkowski et al. 1997; Dixon et al. 1998). However, EGRET lacked the sensitivity and angular resolution to reveal the de- tailed structure of gamma-ray emission toward the inner Galaxy. The Fermi Gamma-ray Space Telescope provides greatly improved data up to $\sim 100 \mathrm{GeV}$, with sufficient angular resolution to map out interesting structures. ${ }^{3}$

At lower energies, the ROSAT All-Sky Survey at $1.5 \mathrm{keV}$ (Snowden et al. 1997) revealed a biconical X-ray structure over the inner tens of degrees around the Galactic center (GC), later interpreted as a superwind bubble (SWB) with energetics of the order of $10^{54}-10^{55} \mathrm{erg}$ (Sofue 2000a; Bland-Hawthorn \& Cohen 2003a). On smaller scales, the Midcourse Space Experiment combined with IRAS data also confirms the existence of a limbbrightened bipolar structure, the so-called Galactic center lobe (GCL), with origin at the GC on the degree scale (see, e.g., Law 2010, for a summary of multiwavelength observations of GCL). The inferred energy injection of both these bipolar structures, despite their different scales, is $\sim 10^{54}-10^{55} \mathrm{erg}$, with an estimated age of $\sim 10^{6} \mathrm{yr}$ for the GCL and $\sim 10^{7} \mathrm{yr}$ for the SWB. Several Galactic center shells, tens of parsecs in size, have been found with total energy of order $\sim 10^{51} \mathrm{erg}$ (Sofue 2003). These shells and filaments are claimed to originate from one or more episodes of rapid energy release.

\subsection{Microwave Excess: the WMAP Haze}

Beyond direct evidence of shell structures, microwave observations also provide intriguing indications of energy release toward the GC.

At tens of GHz, the Wilkinson Microwave Anisotropy Probe $(W M A P)^{4}$ provides sensitive degree resolution full sky maps of diffuse microwave emission. By subtracting templates including Galactic $\mathrm{H} \alpha$, Haslam $408 \mathrm{MHz}$ soft synchrotron, and

\footnotetext{
3 See http://fermi.gsfc.nasa.gov/ssc/data/.

4 http://map.gsfc.nasa.gov/
} 
thermal dust emission to remove the different known emission mechanisms in these maps, a microwave residual excess (named "the microwave haze") with spherical (non-disklike) morphology about $\sim 4 \mathrm{kpc}$ in radius toward the GC (visible up to at least $|b| \approx 30^{\circ}$ ) has been recognized (Finkbeiner 2004a). It has a spectrum of about $I_{v} \sim v^{-0.5}$, harder than typical synchrotron, but softer than free-free. The microwave haze was later interpreted as synchrotron emission from a hard spectrum of $e^{-}$ cosmic rays (CRs). Other hypotheses such as free-free, spinning dust, or thermal dust have failed to explain its morphology, spectrum, or both (Finkbeiner 2004b; Dobler \& Finkbeiner 2008). However, the most recent WMAP 7-year data have not detected the haze polarization predicted by the synchrotron hypothesis (Gold et al. 2010), implying either heavily tangled magnetic fields, or an alternative emission mechanism. With that caveat in mind, we will assume the WMAP haze is synchrotron and consider the implications.

\subsection{A Hard Electron CR Spectrum}

A simple model, in which the electron CRs that form the haze have diffused from supernova (SN) shocks in the disk, cannot fully explain the data for standard diffusion assumptions. The 23-33 GHz spectrum of the haze synchrotron is as hard as that generated from shocks, and it seems extremely unlikely that these electrons can diffuse several kpc from the disk without significant softening of the spectrum. The synchrotron cooling timescale for CR electrons emitting at frequency $v$ is $\tau_{\text {syn }} \approx$ $10^{6} B_{100}^{-3 / 2} v_{\mathrm{GHz}}^{-1 / 2} \mathrm{yr}$, where $B_{100}=B / 100 \mu \mathrm{G}$ (Thompson et al. 2006). Besides the hard spectrum, it is difficult to form the distinctly non-disklike morphology of the haze with any population of sources concentrated in the disk (as is believed to be true of $\mathrm{SNe}$ ).

The presence of a distinct component of diffuse hard $e^{-} \mathrm{CR}$ far off the plane is intriguing in itself, and has motivated proposals where the haze is generated by pulsars, other astrophysical processes, or the annihilation of dark matter (Hooper et al. 2007; Cholis et al. 2009b; Zhang et al. 2009; Harding \& Abazajian 2010; Kaplinghat et al. 2009; McQuinn \& Zaldarriaga 2010; Malyshev et al. 2010). Other indications of excess electronic activity in the Milky Way may be found in recent measurements of local electron and positron CRs. The ATIC, Fermi, and High Energy Stereoscopic System (H.E.S.S.) experiments have observed a hardening in the $e^{+}+e^{-}$spectrum at $20-1000 \mathrm{GeV}$ (Chang et al. 2008; Aharonian 2009; Abdo et al. 2009), with an apparent steepening at $\sim 1 \mathrm{TeV}$, and the PAMELA experiment has measured a rising positron fraction above $10 \mathrm{GeV}$. Taken together, these measurements imply a new source of hard electrons and positrons, which may be related to the WMAP haze. The coexistence of ROSAT X-ray bipolar features and the WMAP haze toward the inner Galaxy also suggests the interesting possibility of a common physical origin for these signals.

\subsection{Inverse Compton Excess from Fermi-LAT}

Fortunately, if the WMAP haze is synchrotron radiation from a hard electron population located around the GC, the same CRs would also produce IC scattered gammas, allowing an independent probe of the $\mathrm{CR}$ population. IC photons provide valuable complementary information about the spatial distribution of the $e^{-} \mathrm{CR}$ (given a model for the interstellar radiation field (ISRF)), which in turn can constrain hypotheses about their origin. The unprecedented angular resolution and sensitivity of Fermi-LAT allows us to probe the gamma-ray counterpart to the microwave haze in detail for the first time.
Previous work employing the first year Fermi-LAT data isolated a spectrally hard "gamma-ray haze" with similar morphology to the WMAP microwave haze (Dobler et al. 2010). In this work, we show that the Fermi-LAT sky maps constructed from 1.6 yr data (600 days) reveal two large gamma-ray lobes, extending $50^{\circ}$ above and below the GC, with a width of about $40^{\circ}$ in longitude. These two "bubble"-like structures have relatively sharp edges and are symmetric with respect to the galactic plane and the minor axis of the galactic disk. The gamma-ray signal reveals similar morphology to the WMAP haze, and is also suggestive of a common origin with features in the ROSAT X-ray maps at $1.5 \mathrm{keV}$ toward the GC.

As we will discuss, the sharp edges, bilobular shape, and apparent centering on the GC of these structures suggest that they were created by some large episode of energy injection in the $\mathrm{GC}$, such as a past accretion onto the central $\mathrm{BH}$, or a nuclear starburst in the last $\sim 10 \mathrm{Myr}$. It is well known that the GC hosts a massive $\mathrm{BH}$ and massive clusters of recently formed stars (Paumard et al. 2006). Either of these could potentially provide the necessary energy injection by driving large-scale galactic winds or producing energetic jets; we will outline some of the advantages and disadvantages of each scenario.

\subsection{Structure of This Paper}

In Section 2, we briefly review the Fermi-LAT data and our data analysis procedure. In Section 3, we show the $1.6 \mathrm{yr}$ Fermi data maps, reveal the Fermi bubble features and show that they are robust when different models for the expected Galactic diffuse emission are subtracted. We characterize the morphology of the bubbles in some detail and employ regression template fitting to reveal a hard, spatially uniform spectrum for the gamma-ray emission associated with the bubbles. In Section 4, we show that features in the ROSAT soft X-rays and the WMAP microwave haze are spatially correlated with the Fermi bubbles, and the WMAP haze and Fermi bubbles are consistent with being produced from the same electron CR population (by synchrotron and IC respectively). Section 5 presents our understanding based on the analysis in Sections 3 and 4. Section 6 discusses possible scenarios to produce the Fermi gamma-ray bubbles. Section 7 focuses on the origin of the electron CRs and the challenges in explaining the spectral and spatial profiles of the gamma-ray emission from the bubbles. We discuss the implications of the Fermi bubbles for several topics of interest in Section 8, and give our conclusions and suggest future work in Section 9.

\section{FERMI DATA AND MAP MAKING}

The Large Area Telescope (LAT; see Gehrels \& Michelson 1999; Atwood et al. 2009; also see the Fermi homepage ${ }^{5}$ ) is a pair-conversion telescope consisting of 16 modules of tungsten and silicon-strip trackers, on top of a calorimeter with a thickness of seven radiation lengths. It has a scintillating anti-coincidence detector that covers the tracker array, and a programmable trigger and data acquisition system. The LAT provides a wide field of view, and covers the energy range from about $30 \mathrm{MeV}-300 \mathrm{GeV}$.

The spacecraft occupies a low Earth orbit with an inclination of $25^{\circ} .6$. The field of view is so wide that the entire sky may be covered in two orbits by rocking the spacecraft north of zenith on one orbit and south of zenith on the next. This scan strategy

\footnotetext{
5 http://fermi.gsfc.nasa.gov/
} 
exposes the LAT to atmospheric gammas at high zenith angles. We make use of only events designated "Class 3" (P6_V3 diffuse class) by the LAT pipeline with a zenith angle cut of $105^{\circ}$.

The events are binned into a full sky map using HEALPix, a convenient iso-latitude equal-area full-sky pixelization widely used in the cosmic microwave background (CMB) community. ${ }^{6}$ Spherical harmonic smoothing is straightforward in this pixelization, and we smooth each map by the appropriate kernel to obtain a Gaussian point-spread function (PSF) of $2^{\circ}$ FWHM. Because the PSF of the initial map must be smaller than this, at energies below $1 \mathrm{GeV}$ we use only front-converting events (which have a smaller PSF). A larger smoothing scale would help improve $\mathrm{S} / \mathrm{N}$, but a relatively small smoothing scale is necessary to see sharp features (such as the bubble edges). Furthermore, for the comparisons and linear combination analysis described in, e.g., Section 3.1.3. it is necessary to smooth the maps at each energy to a common PSF. We generate maps from $1^{\circ}-4^{\circ}$, and find that a FWHM of $2^{\circ}$ works well for our purposes. See Dobler et al. (2010) for details on map construction, smoothing, masking, and for instructions on how to download the maps.

Our current gamma-ray maps (v2_3) constructed from the Fermi data have greater signal/noise compared to the previously released v1_0 maps. They contain photon events from mission times 239557417.494176 to 291965661.204593, for about 606 days or 1.66 years of data (rather than 1 year). We refer to these as the "1.6 year maps." As in Dobler et al. (2010), we construct maps of front-converting and back-converting events separately, smooth to a common PSF, and then combine them. The point source subtraction has improved: instead of interpolating over every source in the 3-month catalog, we use the 1 -year catalog, ${ }^{7}$ and subtract each point source from the maps in each energy bin, using estimates of the PSF from the Fermi science tools. ${ }^{8}$ For the 200 brightest and 200 most variable sources, the subtraction is noticeably imperfect, so we interpolate over the core of the PSF after subtracting the best estimate. We take care to expand the mask for very bright sources (Geminga, 3C 454.3, and LAT PSR $\mathrm{J} 1836+5925)$. The resulting map is appropriate for diffuse work at $|b|>3^{\circ}$. At $|b|<3^{\circ}$ the maps are severely compromised by the poor subtraction and interpolation over a large number of point sources. Further details of the map processing may be found in Appendix B of Dobler et al. (2010). The v2_3 maps used in this work and color versions of the map are available for download. ${ }^{9}$

\section{FERMI BUBBLES}

\subsection{Diffuse Galactic Emission Models}

At low $(\sim 1 \mathrm{GeV})$ energies, and close to the Galactic plane, the gamma rays observed by Fermi are dominated by photons from the decay of $\pi^{0}$ particles, produced by the collisions of $\mathrm{CR}$ protons with ambient ionized gas and dust in the interstellar medium (ISM). Collisions of high-energy CR electrons with the ISM (primarily protons, but also heavier nuclei) produce bremsstrahlung radiation. The Fermi all-sky gamma-ray maps in different energy bands are shown in Figure 1. In order

\footnotetext{
6 HEALPix software and documentation can be found at http://healpix.jpl.nasa.gov, and the IDL routines used in this analysis are available as part of the IDLUTILS product at http://sdss3data.lbl.gov/software/idlutils.

7 Available from http://fermi.gsfc.nasa.gov/ssc/data.

8 See http://fermi.gsfc.nasa.gov/ssc/data/analysis/documentation/.

9 Available at http://fermi.skymaps.info.
}

to uncover the Fermi bubble features better, significant $\pi^{0}$ emission, bremsstrahlung, and IC emission from the Galactic disk must be removed. We take three approaches for the foreground removal. One is to use the Fermi Diffuse Galactic Model provided by the Fermi team ${ }^{10}$ (Section 3.1.1). The second approach employs a linear combination of templates of known emission mechanisms (Section 3.1.2), using existing maps from multiwavelength observations and/or constructed geometric templates. The third approach is taking advantage of the lower energy band $0.5-1.0 \mathrm{GeV}$ Fermi map to form a template of a diffusion emission model (Section 3.1.3).

\subsubsection{Fermi Diffuse Galactic Model}

The Fermi diffuse Galactic model ${ }^{11}$ is a comprehensive model of Galactic gamma-ray emission from the ISM, and serves as a background estimate for point source removal. This model is based on template fits to the gamma-ray data, and includes an IC component generated by the GALPROP CR propagation code. GALPROP calculates the steady-state solution to the diffusion-energy-loss equation, given the threedimensional gas distribution, interstellar radiation field, $B$ field model, CR diffusion assumptions, and many other input parameters (Strong \& Moskalenko 1999; Strong et al. 2009, 2007). The model is constrained by gamma-ray and microwave observations, locally measured CR spectra, etc. By using a well-motivated physical model, one can solve for the spectral and spatial dependence of the injection function, i.e., the $e^{-}$ and $p$ CR primary source spectra, as a function of position and energy. The diffuse model is the key connection between the input assumptions and the observables, and is essential for interpretation of the Fermi-LAT data. It is important to make it as complete as possible.

In this model, the $\pi^{0}$ emission is modeled with maps of interstellar gas: $\mathrm{H}$ I from the Leiden/Argentine/Bonn (LAB) Galactic Survey (Kalberla et al. 2005) and CO from the CfA composite CO survey (Dame et al. 2001). Because the $\pi^{0}$ emission is a function of both the gas density and the proton $\mathrm{CR}$ density, which varies with Galactocentric radius, it is desirable to allow the emissivity of the gas to vary. Both the $\mathrm{H}$ I and $\mathrm{CO}$ surveys contain velocity information, which allows separation into six Galactocentric annuli (rings) with boundaries at 4.0, $5.5,7.0,10.0,16.5$, and $50 \mathrm{kpc}$. The spectrum of each is allowed to float, with the constraint that the sum of the rings along each line of sight approximates the observed signal. This freedom also allows for varying amounts of bremsstrahlung (with varying spectrum) which also scales with the ISM density. The contribution from IC is modeled with GALPROP as described above, and included in the ring fit. ${ }^{12}$

This procedure provides a diffuse model that faithfully reproduces most of the features of the diffuse Galactic emission. One shortcoming is the existence of "dark gas" (Grenier et al. 2005), clouds with gamma-ray emission that do not appear in the $\mathrm{H}$ I and CO surveys. These features are seen in dust maps (Schlegel et al. 1998) and may simply be molecular H clouds underabundant in $\mathrm{CO}$.

The Fermi diffuse model is primarily intended as a background for point source detection, and comes with a number of

\footnotetext{
10 See http://fermi.gsfc.nasa.gov/ssc/data/access/lat/BackgroundModels.html

11 Available from http://fermi.gsfc.nasa.gov/ssc/data. The version of the diffuse model we use is gll.iem.v02.

12 A description of this model is available at http://fermi.gsfc.nasa.gov/ssc/data/access/lat/ring_for_FSSC_final4.pdf.
} 

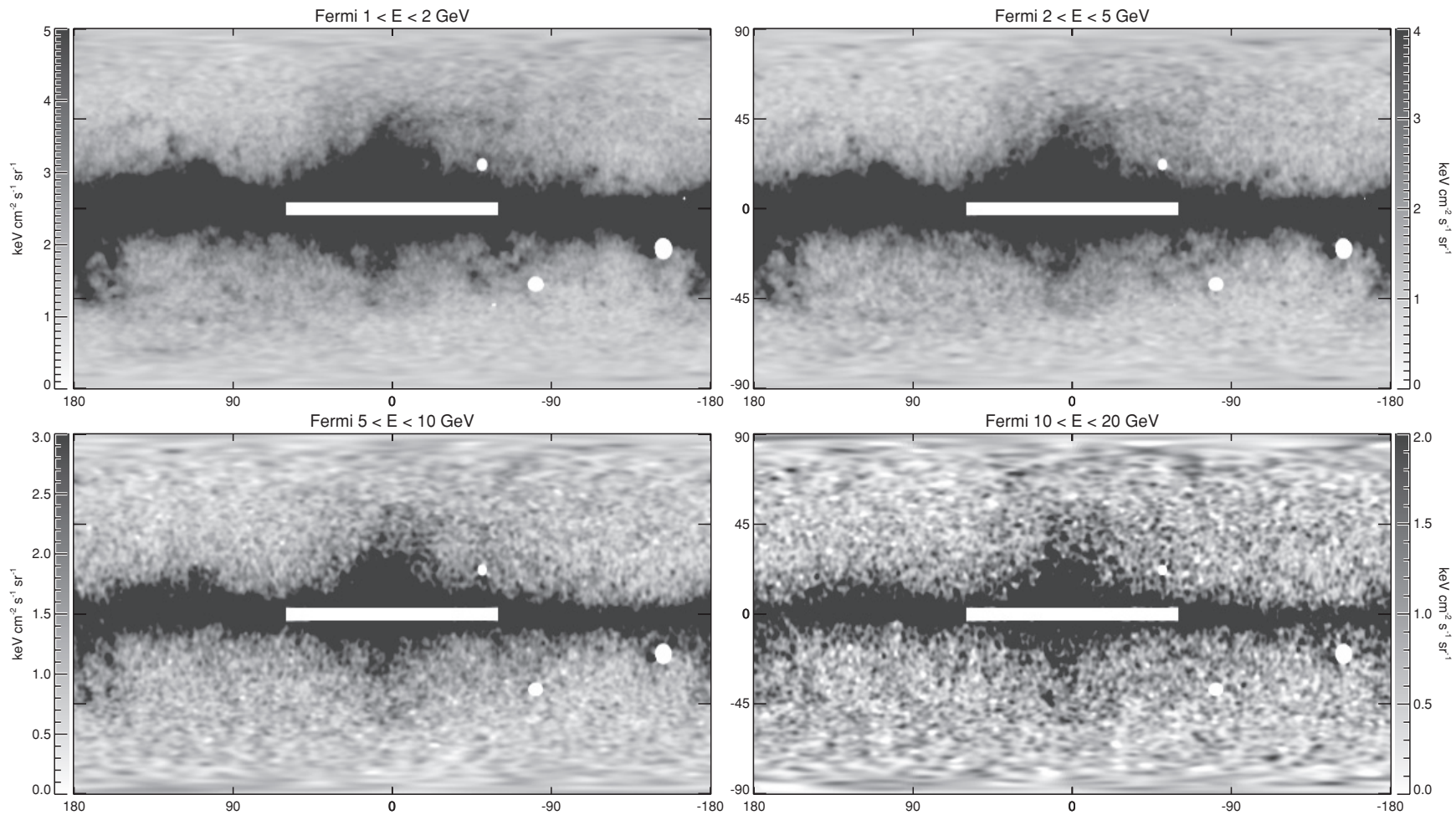

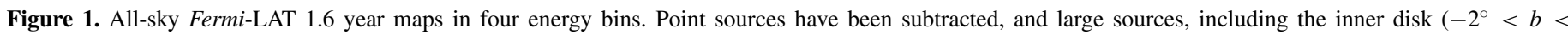
$2^{\circ},-60^{\circ}<\ell<60^{\circ}$ ), have been masked.

caveats. However these caveats apply mainly near the Galactic plane, and at $E>50 \mathrm{GeV}$. It is nevertheless useful for qualitatively revealing features in the diffuse emission at high latitude. In Figure 2, we show the residual maps after subtracting the Fermi diffuse Galactic model in different energy bins. A double-lobed bubble structure is clearly revealed, with similar morphology in the different energy bins. We note that the bubble is neither limb brightened nor centrally brightened, consistent with a flat projected intensity distribution.

\subsubsection{Simple Template-based Diffuse Galactic Model}

Since the dominant foreground gamma rays originate from $\pi^{0}$ gammas produced by CR protons interacting with the ISM, the resulting gamma-ray distribution should be morphologically correlated with other maps of spatial tracers of the ISM. A good candidate is the Schlegel, Finkbeiner, \& Davis (SFD) map of Galactic dust, based on $100 \mu \mathrm{m}$ far IR data (Schlegel et al. 1998). The $\pi^{0} /$ bremsstrahlung gamma-ray intensity is proportional to the ISM density $\times$ the CR proton/electron density integrated along the line of sight. As long as the CR proton/electron spectrum and density are approximately spatially uniform, the ISM column density is a good tracer of $\pi^{0} /$ bremsstrahlung emission. The dust map has some advantages over gas maps: there are no problems with self-absorption, no concerns about "dark gas" (Grenier et al. 2005), and the SFD dust map has sufficient spatial resolution (SFD has a spatial resolution of $6^{\prime}$, and LAB is $\left.36^{\prime}\right)$. On the other hand, SFD contains no velocity information, so it is impossible to break the map into Galactocentric rings. Nevertheless, it is instructive to employ the SFD map to build a very simple foreground model. The goal is to remove foregrounds in a fashion that reveals the underlying structure with as few physical assumptions as possible. We will compare the resulting residuals using this simple diffuse model with those using the Fermi diffuse Galactic model.
As an example, we reveal the Fermi bubble structure from 1-5 GeV Fermi-LAT 1.6 yr data in Figure 3. We use the SFD dust map as a template of the $\pi^{0}$ gamma foreground. The correlation between Fermi and SFD dust is striking, and the most obvious features are removed by this subtraction (top row in Figure 3). This step makes the bubbles above and below the GC easily visible. The revealed bubbles are not aligned with any structures in the dust map, and cannot plausibly be an artifact of that subtraction.

Next, a simple disk model is subtracted (Figure 3, middle row). The purpose of this subtraction is to reveal the structure deeper into the plane, and allow a harder color stretch. The functional form is $(\csc |b|)-1$ in latitude and a Gaussian $\left(\sigma_{\ell}=30^{\circ}\right)$ in longitude. The disk model mostly removes the IC gamma rays produced by $\mathrm{CR}$ electrons interacting with the ISRF including CMB, infrared, and optical photons; as discussed previously, such electrons are thought to be mostly injected in the Galactic disk by SN shock acceleration before diffusing outward.

Finally, we fit a simple double-lobed geometric bubble model with flat gamma-ray intensity to the data, to remove the remaining large-scale residuals toward the GC (Figure 3, bottom row). In this model, we identify the approximate edges of the two bubble-like structures toward the GC in the bottom left panel (shown with dashed green line in right panels of Figure 4). We then fill the identified double-lobed bubble structure with uniform gamma-ray intensity, as a template for the "Fermi bubbles" (bottom right panel of Figure 3). If the Fermi bubbles constitute the projection of a three-dimensional two-bubble structure symmetric to the Galactic plane and the minor axis of the Galactic disk, taking the distance to the GC $R_{\odot}=8.5 \mathrm{kpc}$, the bubble centers are approximately $10 \mathrm{kpc}$ away from us and $5 \mathrm{kpc}$ above and below the GC, extending up to roughly $10 \mathrm{kpc}$ as the most distant edge from GC has 

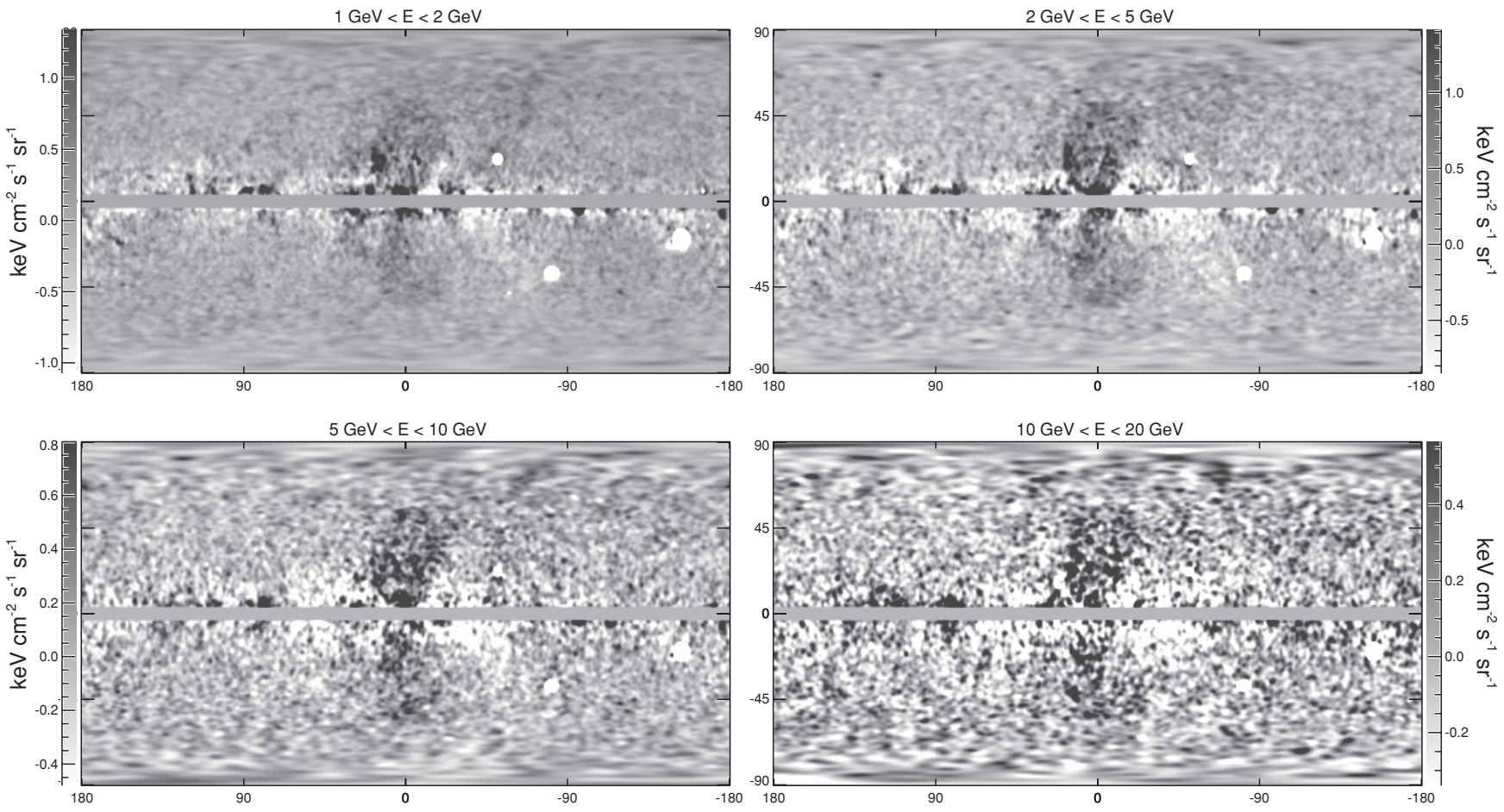

Figure 2. All-sky residual maps after subtracting the Fermi diffuse Galactic model from the LAT 1.6 year maps in four energy bins (see Section 3.1.1). Two bubble structures extending to $b \pm 50^{\circ}$ appear above and below the GC, symmetric about the Galactic plane.

$|b| \sim 50^{\circ}$. No structures like this appear in GALPROP models, and in fact GALPROP is often run with a box height smaller than this. Because the structures are so well centered on the GC, they are unlikely to be local.

In Figure 4, we show the full sky residual maps at $1-5 \mathrm{GeV}$ and $5-50 \mathrm{GeV}$ after subtracting the SFD dust and the disk model to best reveal the Fermi bubble features. Although photon Poisson noise is much greater in the $5-50 \mathrm{GeV}$ map, we identify a Fermi bubble structure morphologically similar to the structure in the $1-5 \mathrm{GeV}$ map, present both above and below the Galactic plane.

In Figure 5, we show the full sky maps at $1-5 \mathrm{GeV}$ with the zenithal equal area (ZEA) projection with respect to both north pole and south pole. We found no interesting features appear near the poles.

\subsubsection{Low-energy Fermi Map as a Diffuse Galactic Model}

In Figure 6, we show the $0.5-1 \mathrm{GeV}$ and $2-50 \mathrm{GeV}$ residual maps after subtracting only the SFD dust map as a template of foreground $\pi^{0}$ gammas. The residual maps should be dominated by IC emission from CR electrons interacting with the ISRF. We use the $0.5-1 \mathrm{GeV}$ maps as a template of IC emission from high energy electrons scattering starlight, and subtract the template from higher energy maps (the lower panels of Figure 6). The Fermi bubble structures are clearly revealed. We thus conclude that the Fermi bubbles are mostly from high energy electron CRs IC scattering on CMB photons, and IR photons at higher energies (see Section 5 for more discussion). By comparing the Fermi diffuse Galactic model subtraction (Figure 2) and our simple template model subtraction (Figure 4), we find that the bubble structures are robust to quite different foreground subtractions. It is difficult to see how such emission could arise — especially with sharp edges (Section 3.2.1) —as an artifact of these subtractions.

\subsection{Fermi Bubbles: Morphology}

\subsubsection{Morphological Features}

In the right panels of Figure 4, we illustrate the edges of the Fermi bubbles and some other features. We find that the Fermi bubbles have distinct sharp edges, rather than smoothly falling off as modeled in Dobler et al. (2010). Besides the two bubbles symmetric with respect to the Galactic plane, we find one giant northern arc that embraces half of the north bubble, that extends from the disk up to $b \sim 50$, with $\ell$ ranging from roughly $-40^{\circ}$ to $0^{\circ}$. It has a brighter and sharper outer edge in the $1-5 \mathrm{GeV}$ map. On a even larger scale, we identify a fainter structure extended up to $b \sim 80^{\circ}$, with $\ell$ ranging from roughly $-80^{\circ}$ to $50^{\circ}$. We will show in Section 4 that this large extended structure corresponds to the North Polar Spur emission associated with Loop I (as seen, for example, in the Haslam $408 \mathrm{MHz}$ map Haslam et al. 1982). We will discuss the possible relation of the Fermi bubble, the northern arc, and the Loop I feature in Section 8 . In the $1-5 \mathrm{GeV}$ map, we also identify a "donut-like" structure in the south sky with $b$ ranging from roughly $-35^{\circ}$ to $0^{\circ}$ and $\ell$ from roughly $0^{\circ}$ to $40^{\circ}$. The coordinates of the Fermi bubble edges, northern arc, Loop I, and the "donut" feature identified from the 1-5 GeV map are listed in Table 1.

In Figure 7, we compare the Fermi bubble morphology in different energy bins. We show the difference of the 1-2 and 2-5 GeV residual maps in the upper panels; each residual map is the result of subtracting the SFD dust map and the simple disk model to best reveal the Fermi bubbles. The difference maps between the 1-5 and 5-50 GeV maps are shown in the lower panels. The bubble features almost disappear in the difference maps, indicating that different parts of the Fermi bubbles have similar spectra.

To study the sharp edges of the bubbles at high latitude more carefully, we examine the (projected) intensity profiles along arcs of great circles passing through the estimated centers of 

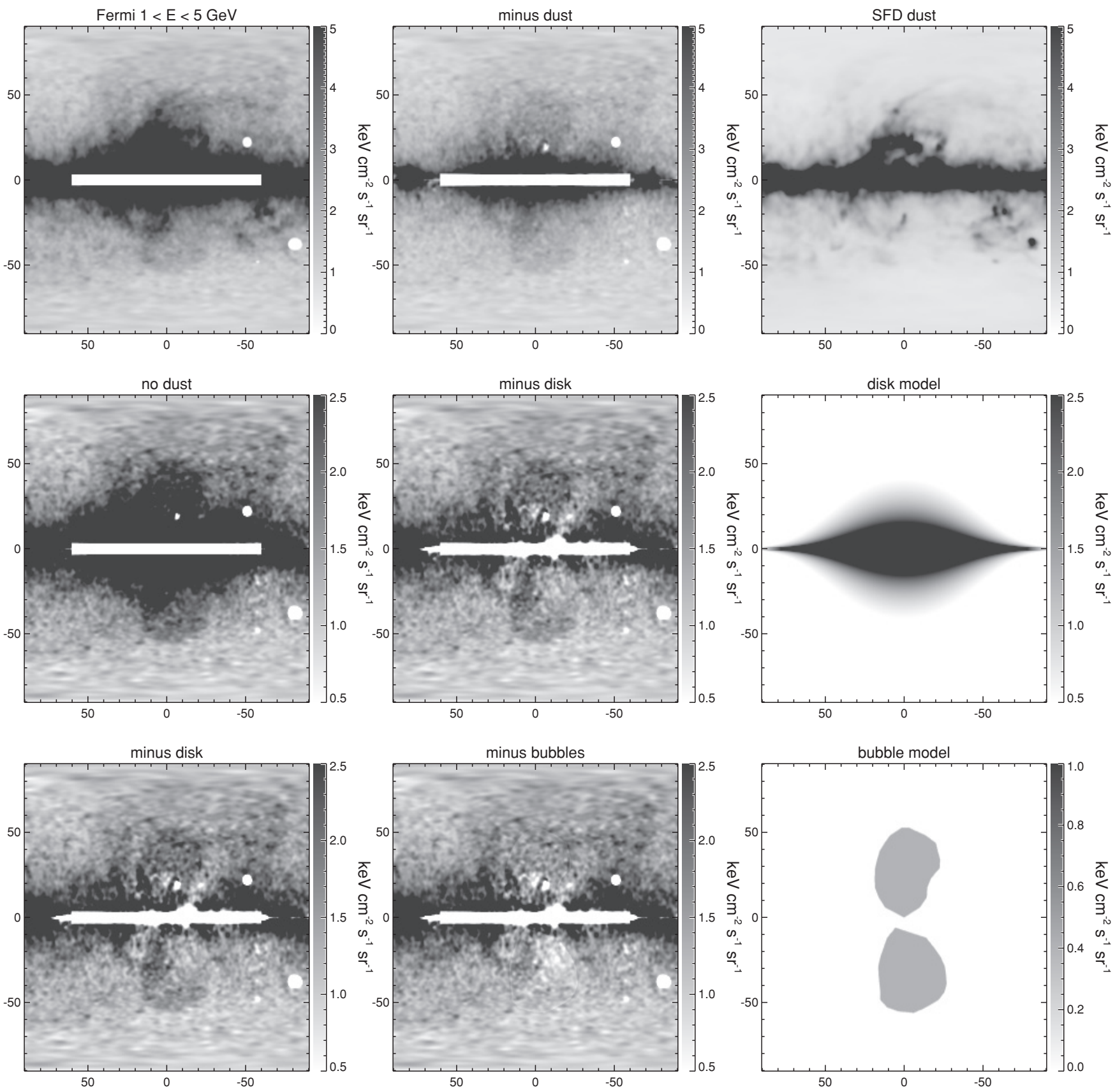

Figure 3. Template decomposition of the Fermi-LAT 1.6 year $1-5 \mathrm{GeV}$ map (see Section 3.1.2). Top left: point source subtracted 1-5 GeV map, and large sources, including the inner disk $\left(-2^{\circ}<b<2^{\circ},-60^{\circ}<\ell<60^{\circ}\right)$, have been masked. Top middle: the $1-5 \mathrm{GeV}$ map minus SFD dust map (top right panel) which is used as a template of $\pi^{0}$ gammas. Middle row: The left panel is the same as the top middle panel but stretched $2 \times$ harder. The middle panel subtracts a simple geometric disk template (shown in the right panel), representing mostly IC emission, to reveal features close to the GC. Two large bubbles are apparent (spanning $-50^{\circ}<b<50^{\circ}$ ). Bottom row: The left panel is the same as the middle panel of the second row. Finally we subtract a simple bubble template (right panel), with a shape derived from the edges visible in the maps, and uniform projected intensity. After subtracting the bubble template, the two bubbles features have nearly vanished (bottom middle panel), indicating a nearly flat intensity for the Fermi bubbles.

the north and south bubbles, and intersecting the bubble edge (as defined in Figure 4) at $|b|>28^{\circ}$. Along each such ray, we define the intersection of the arc with the bubble edge to be the origin of the coordinate system; we then perform an inverse-variance-weighted average of the intensity profile along the rays (as a function of distance from the bubble edge). We subtract a constant offset from the profile along each ray, prior to averaging the rays together, to minimize aliasing of point sources onto the averaged profile, and then add the averaged offset back in at the end. The inverse variance for each data point is obtained from the Poisson errors in the original photon data, prior to any subtraction of point sources or templates (however, the smoothing of the map is taken into account). When the rays are averaged together, the naive inverse variance in the result is multiplied by a factor of the annulus radius (for the points being averaged together) divided by $4 \pi \sigma^{2}$, where $\sigma$ is the $1 \sigma$ value of the PSF, and the annulus width is taken to be $1^{\circ}$ (the spacing between the points along the rays; this is comparable to the smoothing scale, so there may still be unaccounted-for correlations between the displayed errors); this is done to take 

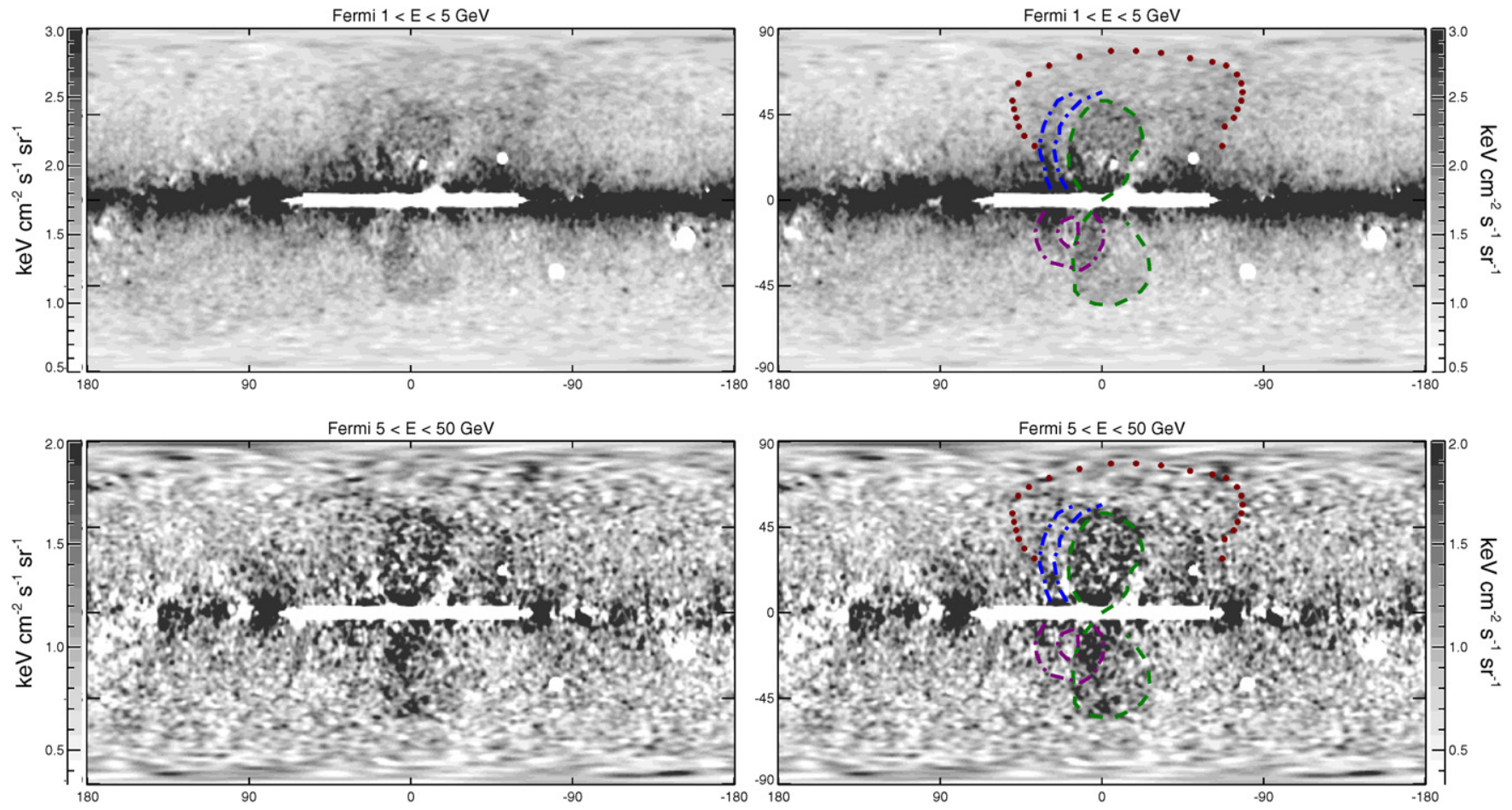

Figure 4. Full sky residual maps after subtracting the SFD dust and disk templates from the Fermi-LAT 1.6 year gamma-ray maps in two energy bins. Point sources are subtracted, and large sources, including the inner disk $\left(-2^{\circ}<b<2^{\circ},-60^{\circ}<\ell<60^{\circ}\right)$, have been masked. Two large bubbles are seen (spanning $\left.-50^{\circ}<b<50^{\circ}\right)$ in both cases. Right panels: Apparent Fermi bubble features marked in color lines, overplotted on the maps displayed in the left panels. Green dashed circles above and below the Galactic plane indicate the approximate edges of the north and south Fermi bubbles respectively. Two blue dashed arcs mark the inner (dimmer) and outer (brighter) edges of the northern arc - a feature in the northern sky outside the north bubble. The red dotted line approximately marks the edge of Loop I. The purple dot-dashed line indicates a tentatively identified "donut" structure.

(A color version of this figure is available in the online journal.)

Table 1

Coordinates Defining the Features Shown in Figures 4 and 7

\begin{tabular}{|c|c|c|c|c|c|c|}
\hline $\begin{array}{l}\text { North Bubble } \\
(\ell, b)(\mathrm{deg})\end{array}$ & $\begin{array}{l}\text { South Bubble } \\
(\ell, b)(\mathrm{deg})\end{array}$ & $\begin{array}{c}\text { North Arc (Outer) } \\
\quad(\ell, b)(\mathrm{deg})\end{array}$ & $\begin{array}{l}\text { North Arc (Inner) } \\
\quad(\ell, b)(\mathrm{deg})\end{array}$ & $\begin{array}{c}\text { Loop I } \\
(\ell, b)(\operatorname{deg})\end{array}$ & $\begin{array}{c}\text { Donut (Outer) } \\
(\ell, b)(\mathrm{deg}) \\
\end{array}$ & $\begin{array}{c}\text { Donut (Inner) } \\
(\ell, b)(\mathrm{deg}) \\
\end{array}$ \\
\hline$(0.0,0.0)$ & $(5.5,-5.0)$ & $(28.5,5.0)$ & $(19.5,5.0)$ & $(37.5,25.0)$ & $(31.9,-5.0)$ & $(16.8,-7.6)$ \\
\hline$(-9.9,5.0)$ & $(10.7,-10.0)$ & $(31.1,10.0)$ & $(23.1,10.0)$ & $(43.2,30.0)$ & $(34.9,-9.0)$ & $(22.0,-9.4)$ \\
\hline$(-14.2,10.0)$ & $(12.9,-15.0)$ & $(31.9,15.0)$ & $(24.9,15.0)$ & $(46.3,35.0)$ & $(37.0,-14.0)$ & $(24.6,-14.0)$ \\
\hline$(-14.5,15.0)$ & $(15.0,-20.0)$ & $(34.0,20.0)$ & $(26.0,20.0)$ & $(47.7,40.0)$ & $(36.3,-19.0)$ & $(23.7,-16.5)$ \\
\hline$(-17.0,20.0)$ & $(16.3,-25.0)$ & $(34.9,25.0)$ & $(26.9,25.0)$ & $(48.9,45.0)$ & $(33.5,-25.0)$ & $(22.4,-18.7)$ \\
\hline$(-22.3,25.0)$ & $(16.0,-30.0)$ & $(33.7,30.0)$ & $(23.7,30.0)$ & $(49.8,50.0)$ & $(28.7,-29.0)$ & $(19.2,-21.2)$ \\
\hline$(-22.6,30.0)$ & $(15.5,-35.0)$ & $(32.5,35.0)$ & $(23.5,35.0)$ & $(46.6,60.0)$ & $(11.5,-33.0)$ & $(13.6,-19.3)$ \\
\hline$(-21.1,35.0)$ & $(15.0,-40.0)$ & $(30.5,40.0)$ & $(20.5,40.0)$ & $(40.6,65.0)$ & $(4.8,-29.0)$ & $(13.2,-14.3)$ \\
\hline$(-19.9,40.0)$ & $(15.0,-45.0)$ & $(27.5,45.0)$ & $(16.5,45.0)$ & $(29.4,70.0)$ & $(-0.4,-20.0)$ & $(13.6,-8.8)$ \\
\hline$(-13.6,45.0)$ & $(10.6,-50.0)$ & $(24.7,50.0)$ & $(11.7,50.0)$ & $(12.5,75.0)$ & $(-1.2,-15.0)$ & $(16.8,-7.6)$ \\
\hline$(-3.0,50.0)$ & $(3.7,-52.5)$ & $(20.0,52.5)$ & $(6.0,52.5)$ & $(-5.0,78.0)$ & $(0.3,-10.0)$ & \\
\hline$(1.5,50.0)$ & $(-6.3,-53.5)$ & $(14.3,55.0)$ & $(0.0,55.0)$ & $(-19.0,78.0)$ & $(5.5,-5.0)$ & \\
\hline$(8.7,45.0)$ & $(-13.8,-50.0)$ & & & $(-33.0,77.0)$ & & \\
\hline$(12.3,40.0)$ & $(-21.8,-45.0)$ & & & $(-49.1,74.0)$ & & \\
\hline$(15.4,35.0)$ & $(-25.3,-40.0)$ & & & $(-61.5,72.0)$ & & \\
\hline$(17.0,30.0)$ & $(-26.7,-35.0)$ & & & $(-69.2,70.0)$ & & \\
\hline$(18.3,25.0)$ & $(-26.3,-30.0)$ & & & $(-75.2,65.0)$ & & \\
\hline$(18.5,20.0)$ & $(-25.6,-25.0)$ & & & $(-77.6,60.0)$ & & \\
\hline$(18.4,15.0)$ & $(-23.0,-20.0)$ & & & $(-78.3,55.0)$ & & \\
\hline$(16.0,10.0)$ & $(-18.8,-15.0)$ & & & $(-77.6,50.0)$ & & \\
\hline \multirow{4}{*}{$(12.0,5.0)$} & $(-13.8,-10.0)$ & & & $(-75.5,45.0)$ & & \\
\hline & & & & $(-73.5,40.0)$ & & \\
\hline & & & & $(-68.3,35.0)$ & & \\
\hline & & & & $(-67.0,25.0)$ & & \\
\hline
\end{tabular}

into account that the number of independent measurements being sampled by the rays can be far less than the number of rays, especially close to the center of the bubbles. This procedure is repeated for all the stages of the template subtraction, using the simple disk template for inverse compton scattering (ICS) for illustration (our conclusions do not depend on this choice). 


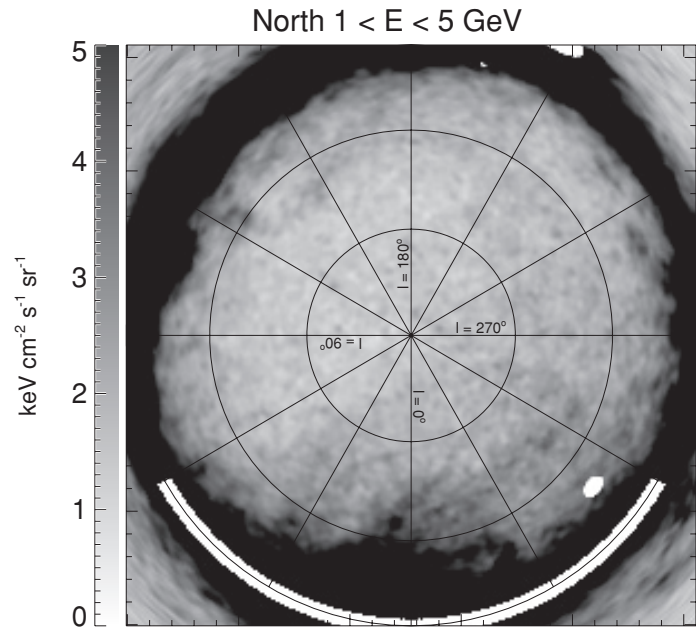

North $1<\mathrm{E}<5 \mathrm{GeV}$, subtracted

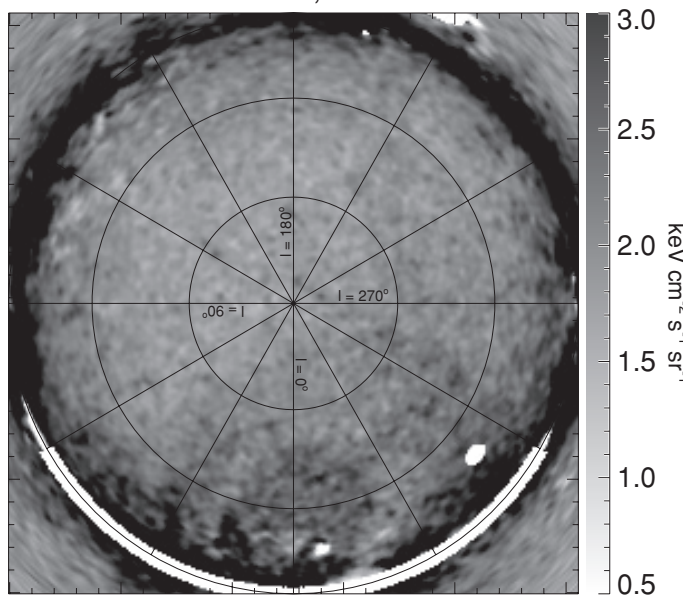

South $1<\mathrm{E}<5 \mathrm{GeV}$, subtracted
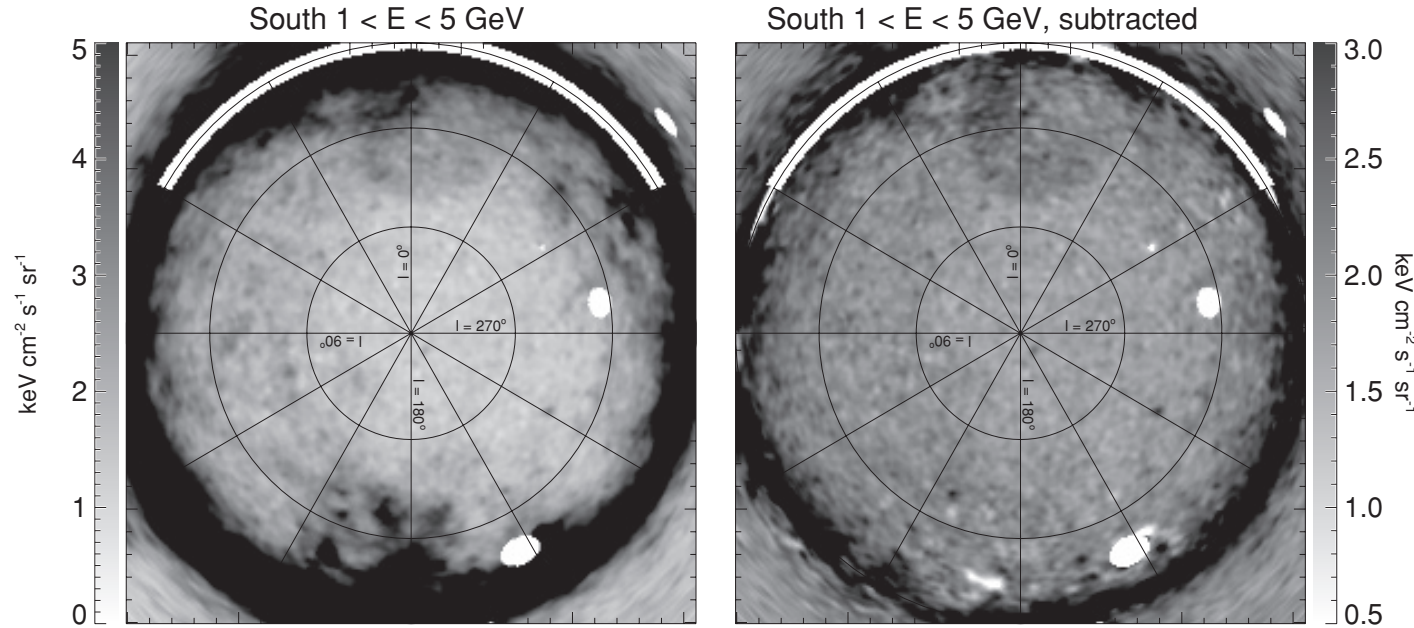

Figure 5. Zenithal equal area (ZEA) projection with respect to both north pole (upper panels) and south pole (lower panels) for the 1-5 GeV energy band before (left panels) and after (right panels) subtracting the SFD dust and simple disk templates to reveal the Fermi bubbles.

The results are shown in Figure 8 for the averaged $(1-2)+$ $(2-5) \mathrm{GeV}$ maps, and the averaged $(5-10)+(10-20) \mathrm{GeV}$ maps. In both energy ranges the edges are clearly visible; in the south, this is true even before any templates are subtracted. The intensity profile of the north bubble is strikingly similar to the profile of the south bubble. For both north and south bubbles, no significant edge brightening or limb brightening of the bubbles is apparent from the profiles, the flux is fairly uniform inside the bubbles.

In Figure 9, we plot the intensity profile as a function of latitude from the south to the north pole. We construct great circle arcs perpendicular to the $l=0$ great circle, extending $10^{\circ}$ in each direction (east and west), and average the emission over each such arc. The flatness of the bubbles with latitude (except possibly close to the Galactic plane) and the sharp edges at high latitude are also apparent here.

We note that the flat intensity of the bubbles is striking. As we show in Figure 10, if we assume that the Fermi bubbles are projected from spherically symmetric three-dimensional bubbles centered above and below the GC, a non-trivial emissivity distribution in the bubble interior is required to produce a flat projected intensity distribution (upper left panel of Figure 10). If the "bubbles" originate from IC scattering, this suggests a rather non-uniform density distribution for the electron CRs, which — combined with a nearly uniform spectral index-pre- sents challenges for many models for the electron injection. The expected intensity profile for a shock generated bubble with a compressed gas shell is shown in the upper right panel; it is noticeably limb brightened, in contrast with observations.

\subsubsection{Spectrum of Gamma-ray Emission}

We now attempt to estimate the spectrum of the gamma rays associated with the Fermi bubbles and the spatial variation of the spectrum. In order to reveal the hardness of the spectrum of the Fermi bubbles, and quantitatively study the intensity flatness of the bubble interiors, we do a careful regression template fitting. First, we maximize the Poisson likelihood of a simple diffuse emission model involving five templates (see Section 3.1.2). In this model, we include the SFD dust map (Figure 3, right panel of the top row) as a tracer of $\pi^{0}$ emission which is dominant (or nearly so) at most energies on the disk and significant even at high latitudes, the simple disk model (Figure 3, right panel of the second row), the bubble template (Figure 3, right panel of the bottom row), the Loop I template (see, e.g., Figure 11, left panel of the top row), and a uniform background as templates to weight the Fermi data properly.

The significant isotropic background is due to extra-galactic emission and charged particle contamination, including heavy 

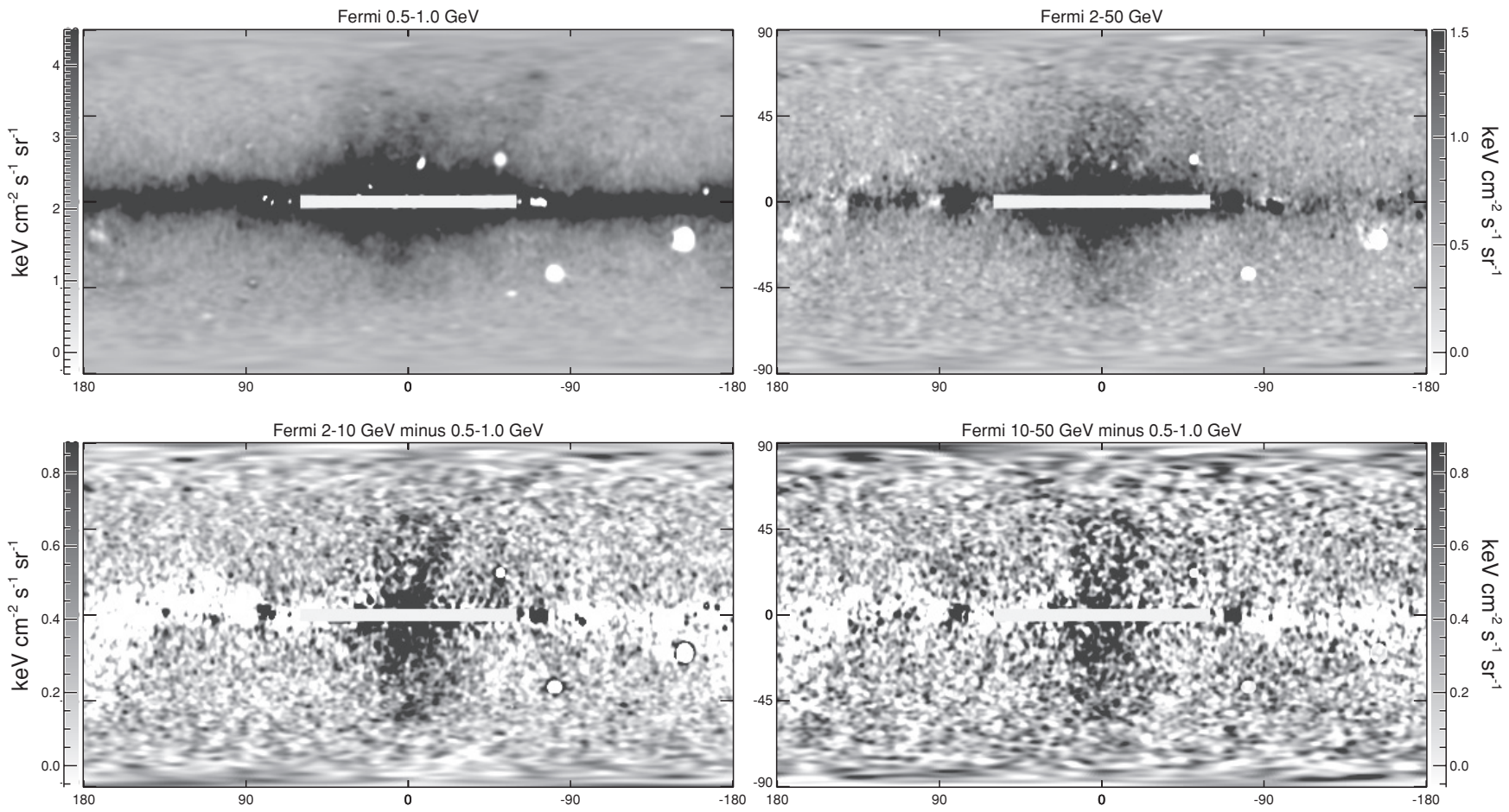

Figure 6. Top left: full sky Fermi-LAT 1.6 year $0.5-1.0 \mathrm{GeV}$ map subtracts the SFD dust map as a template of $\pi^{0}$ gammas. Top right: the same as top left panel, but for energy range $2-50 \mathrm{GeV}$ (note the different gray scale for the two panels). Bottom left: the $2-10 \mathrm{GeV}$ Fermi gamma-ray map subtracting the top left $0.5-1.0 \mathrm{GeV}$ residual map which is used as a template of ICS of starlight. Bottom right: The same as bottom left panel but for $10-50 \mathrm{GeV}$ map subtracting the top left $0.5-1.0 \mathrm{GeV}$ residual map. The Fermi bubble structures are better revealed after subtracting the lower energy $0.5-1.0 \mathrm{GeV}$ residual map with extended disk-like emission.

nuclei at high energies. The Fermi collaboration has measured the extragalactic diffuse emission using additional cuts to reduce charged particle contamination (Abdo et al. 2010b): below $\sim 20 \mathrm{GeV}$, the isotropic contribution in our fits is roughly a factor of two larger than the extragalactic diffuse emission, but has a similar spectral slope. At energies above $\sim 20 \mathrm{GeV}$, the isotropic contribution becomes much harder, which we attribute to charged particle contamination.

For each set of model parameters, we compute the Poisson log likelihood,

$$
\ln \mathcal{L}=\sum_{i} k_{i} \ln \mu_{i}-\mu_{i}-\ln \left(k_{i} !\right),
$$

where $\mu_{i}$ is the synthetic map (i.e., linear combination of templates) at pixel $i$, and $k$ is the map of observed data. The last term is a function of only the observed maps. The $1 \sigma$ Gaussian error is calculated from the likelihood by $\Delta \ln \mathcal{L}=1 / 2$. The error bars are simply the square root of the diagonals of the covariance matrix. We refer to Appendix B of Dobler et al. (2010) for more details of the likelihood analysis. Maps of the models constructed from linear combinations of these five templates, and the residual maps between the Fermi data and the combined templates at different energy bins, are shown in Figure 11. In this fit, we mask out all pixels with Galactic latitude $|b|<30^{\circ}$ (the dashed black line in the residual maps).

Template-correlated spectra for the 5-template fit are shown in Figure 12. The fitting is done with regions of $|b|>30^{\circ}$. For a template that has units (e.g., the SFD dust map is in $E_{B-V}$ magnitudes) the correlation spectrum has obscure units (e.g., gamma-ray emission per magnitude). In such a case, we multiply the correlation spectrum by the average SFD value in the bubble region, defined by the bottom right panel of Figure 3, masking out the $|b|<30^{\circ}$ region. For the uniform, Loop I, and bubble templates (including inner, outer, north, and south), no renormalization is done. These templates are simply ones and zeros (smoothed to the appropriate PSF), so the outer bubble spectrum is simply the spectrum of the bubble shell template shown in Figure 15, not the mean of this template over the whole bubble region. The normalization factors for different templates are listed in Table 2.

In Figure 12, we show spectra for $\pi^{0}$ emission, bremsstrahlung and IC scattering calculated using a sample GALPROP model (tuned to match locally measured protons and anti-protons as well as locally measured electrons at $\sim 20-30 \mathrm{GeV}$ ), as an indication of the expected spectral shapes. The spectra for the SFD and the simple disk template reasonably match the model expectations. The dust map mostly traces the $\pi^{0}$ emission, and the simple disk model resembles a combination of IC and bremsstrahlung emission. The spectrum for emission correlated with the Fermi bubbles is clearly significantly harder than either of these components, consistent with a flat spectrum in $E^{2} d N / d E$. This fact coupled with the distinct spatial morphology of the Fermi bubbles indicates that the IC bubbles are generated by a separate electron component. We also note that the spectrum of the bubble template falls off significantly at energy $\lesssim 1 \mathrm{GeV}$. This feature is robust with respect to the choice of templates. The fitting coefficients and corresponding errors of each template are listed in Table 3. We will discuss some implications of the falling spectrum in Section 4.3.

To demonstrate the robustness of the spectrum we have derived for the Fermi bubbles, we make use of the Fermi 0.5-1 GeV residual map (after subtracting the SFD dust map to largely remove the $\pi^{0}$ gammas) as a template of IC emission, and perform a 4-template fit (Section 3.1.3). These gamma rays mostly originate from IC scattering of a relatively soft population of electrons in the disk, but might also contain 

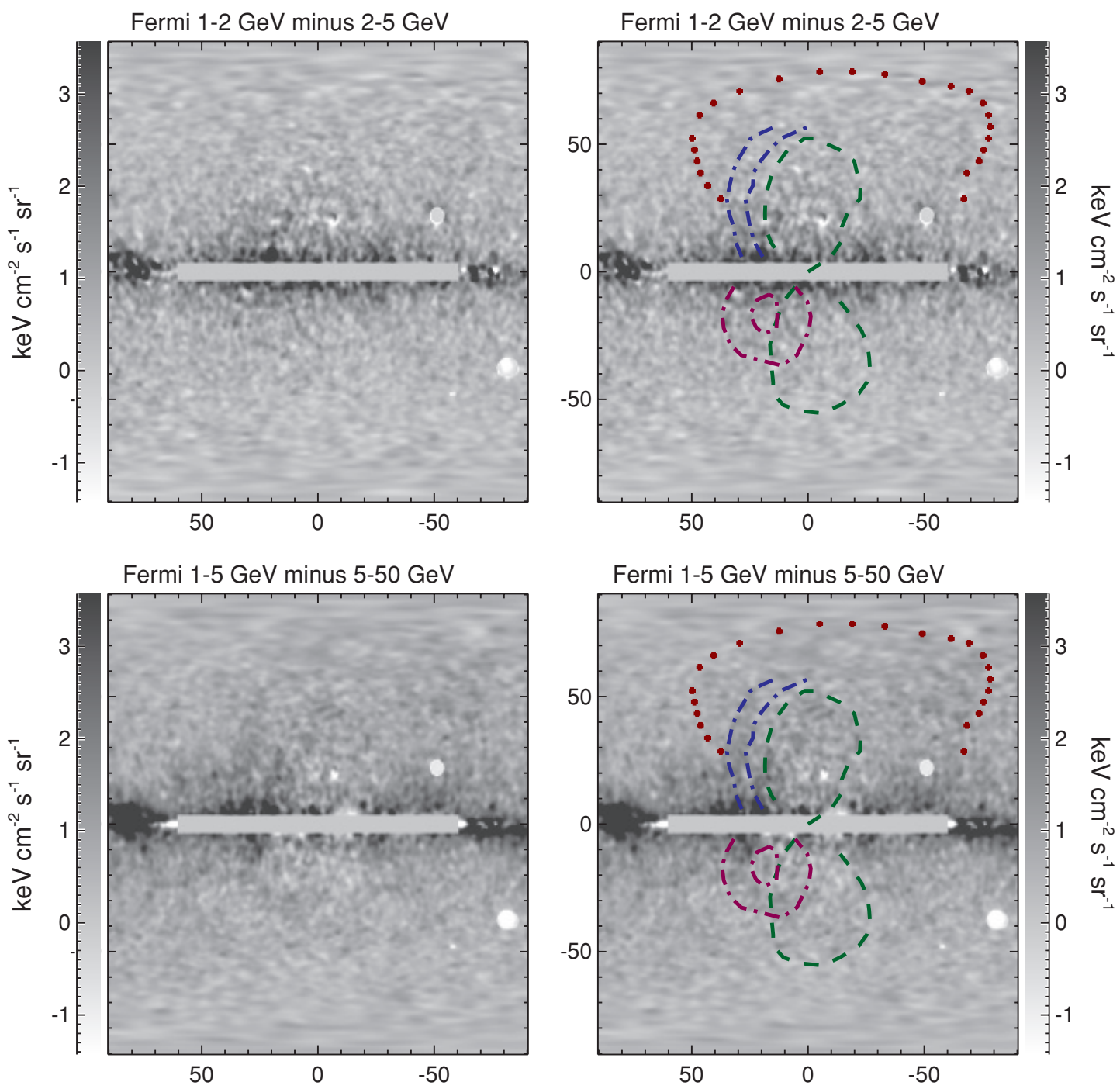

Figure 7. Comparison of the Fermi bubbles between different energy bins. Top left: subtraction of the $2-5 \mathrm{GeV}$ residual map from the 1-2 GeV residual map; each residual map is constructed from the data by regressing out the SFD dust and disk templates to best reveal the Fermi bubbles. The difference map is consistent with Poisson noise away from the masked region, and the bubble features can hardly be recognized, indicating that different spatial regions of the Fermi bubbles have the same spectrum. Top right: the same map as left panel, but with the Fermi bubble features overplotted for comparison. The marked features are the same as those plotted in Figure 4, and are listed in Table 1. Bottom row: same as the upper panels, but subtracting the 5-50 GeV residual map from the 1-5 GeV residual map.

(A color version of this figure is available in the online journal.)

Table 2

Normalization Factors for Different Templates

\begin{tabular}{lccccccccc}
\hline \hline Uniform & SFD & Bubble & North Bubble & South Bubble & Inner Bubble & Outer Bubble & Disk & Loop I & $0.5-1.0 \mathrm{GeV}-\mathrm{SFD}$ \\
\hline 1.0 & 0.084 & 1.0 & 1.0 & 1.0 & 1.0 & 1.0 & 0.292 & 1.0 & 1.198
\end{tabular}

gammas from IC scattering on starlight by a latitudinally extended electron population. We use the SFD dust map as a template for $\pi^{0}$ gammas as previously, and include the uniform background and the bubble template as in the previous 5template fit. The fitting is done with regions of $|b|>30^{\circ}$. For the SFD dust map and the Fermi $0.5-1 \mathrm{GeV}$ IC template, the correlation coefficients are weighted by the mean of each template in the "bubble" region. The resulting model and the difference maps with respect to the Fermi data, at different energy bands, are shown in Figure 13. The residuals are remarkably small. The spectrum is shown in Figure 14. The Fermi $0.5-1 \mathrm{GeV}$ IC template appears to contain a small fraction of $\pi^{0}$ gammas, but the spectral index is consistent with the predicted GALPROP IC component. The fitting coefficients and corresponding errors of each template are listed in Table 4.

By eye, the Fermi bubbles appear to possess north-south symmetry and are close to spatially uniform in intensity (with a hard cutoff at the bubble edges). To test these hypotheses more quantitatively, we split the Fermi bubble template into the inner bubble and outer shell templates (upper row of Figure 15), or alternatively into the north and south bubble templates (lower row of Figure 15). We then repeat the previous 5-template and 4-template fitting procedure involving either the simple disk IC template or the Fermi $0.5-1 \mathrm{GeV}$ IC template, but splitting the bubble template to either inner bubble and outer shell or north and south bubble templates. The goal is to identify variations 

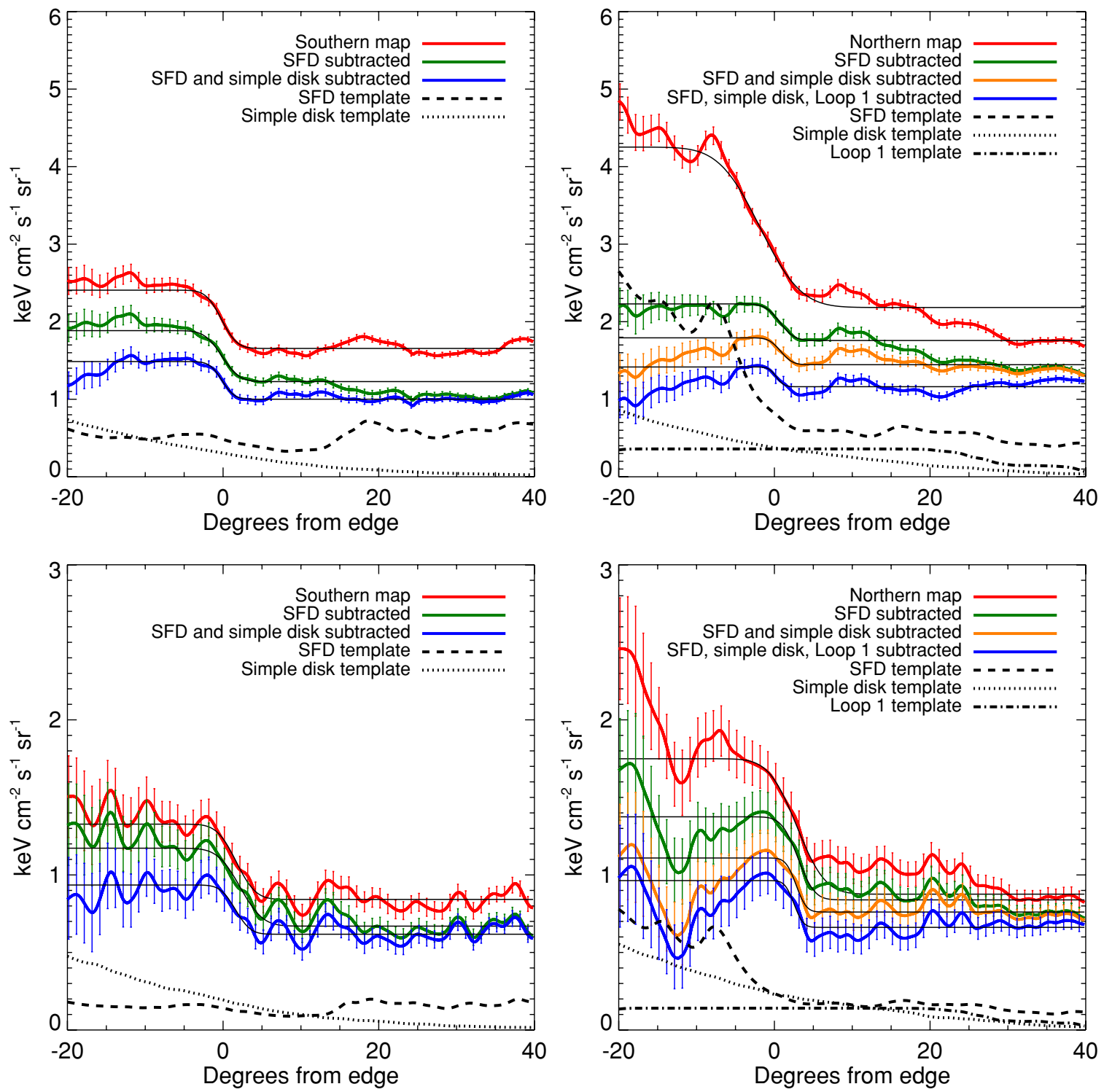

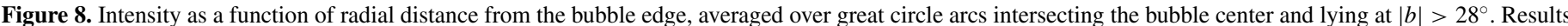

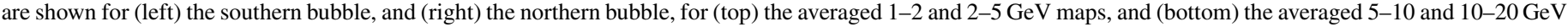

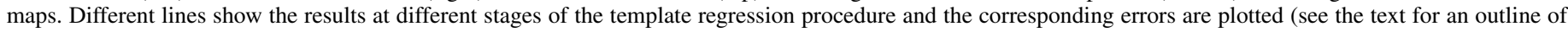
the error analysis).

(A color version of this figure is available in the online journal.)

Table 3

Corresponding Template-fitting Coefficients and Errors in Figure 12

\begin{tabular}{lrccccc}
\hline \hline$E$ Range $(\mathrm{GeV})$ & Energy & Uniform & SFD Dust & Simple Disk & Whole Bubble & Simple Loop I \\
\hline $0.3-0.5$ & 0.4 & $1.681 \pm 0.006$ & $1.201 \pm 0.011$ & $0.689 \pm 0.027$ & $0.035 \pm 0.033$ & $0.487 \pm 0.015$ \\
$0.5-0.9$ & 0.7 & $1.365 \pm 0.007$ & $1.279 \pm 0.012$ & $0.608 \pm 0.030$ & $0.211 \pm 0.037$ & $0.475 \pm 0.016$ \\
$0.9-1.7$ & 1.3 & $1.141 \pm 0.008$ & $1.179 \pm 0.014$ & $0.503 \pm 0.035$ & $0.321 \pm 0.044$ & $0.405 \pm 0.019$ \\
$1.7-3.0$ & 2.2 & $1.034 \pm 0.006$ & $0.876 \pm 0.011$ & $0.393 \pm 0.029$ & $0.436 \pm 0.036$ & $0.376 \pm 0.016$ \\
$3.0-5.3$ & 4.0 & $0.881 \pm 0.008$ & $0.554 \pm 0.013$ & $0.420 \pm 0.034$ & $0.353 \pm 0.043$ & $0.249 \pm 0.018$ \\
$5.3-9.5$ & 7.1 & $0.731 \pm 0.009$ & $0.322 \pm 0.014$ & $0.282 \pm 0.039$ & $0.343 \pm 0.049$ & $0.208 \pm 0.021$ \\
$9.5-16.9$ & 12.7 & $0.563 \pm 0.010$ & $0.193 \pm 0.015$ & $0.251 \pm 0.044$ & $0.205 \pm 0.055$ & $0.092 \pm 0.023$ \\
$16.9-30.0$ & 22.5 & $0.507 \pm 0.012$ & $0.128 \pm 0.018$ & $0.191 \pm 0.053$ & $0.263 \pm 0.068$ & $0.125 \pm 0.029$ \\
$30.0-53.3$ & 40.0 & $0.557 \pm 0.015$ & $0.041 \pm 0.021$ & $0.096 \pm 0.064$ & $0.217 \pm 0.083$ & $0.088 \pm 0.036$ \\
$53.3-94.9$ & 71.1 & $0.628 \pm 0.022$ & $0.020 \pm 0.030$ & $0.183 \pm 0.093$ & $0.251 \pm 0.120$ & $-0.043 \pm 0.048$ \\
$94.9-168.7$ & 126.5 & $0.622 \pm 0.030$ & $0.080 \pm 0.043$ & $0.012 \pm 0.125$ & $0.319 \pm 0.162$ & $-0.091 \pm 0.063$ \\
$168.7-300.0$ & 225.0 & $0.436 \pm 0.038$ & $0.174 \pm 0.061$ & $-0.039 \pm 0.158$ & $-0.015 \pm 0.194$ & $0.083 \pm 0.086$ \\
\hline & & & & & &
\end{tabular}



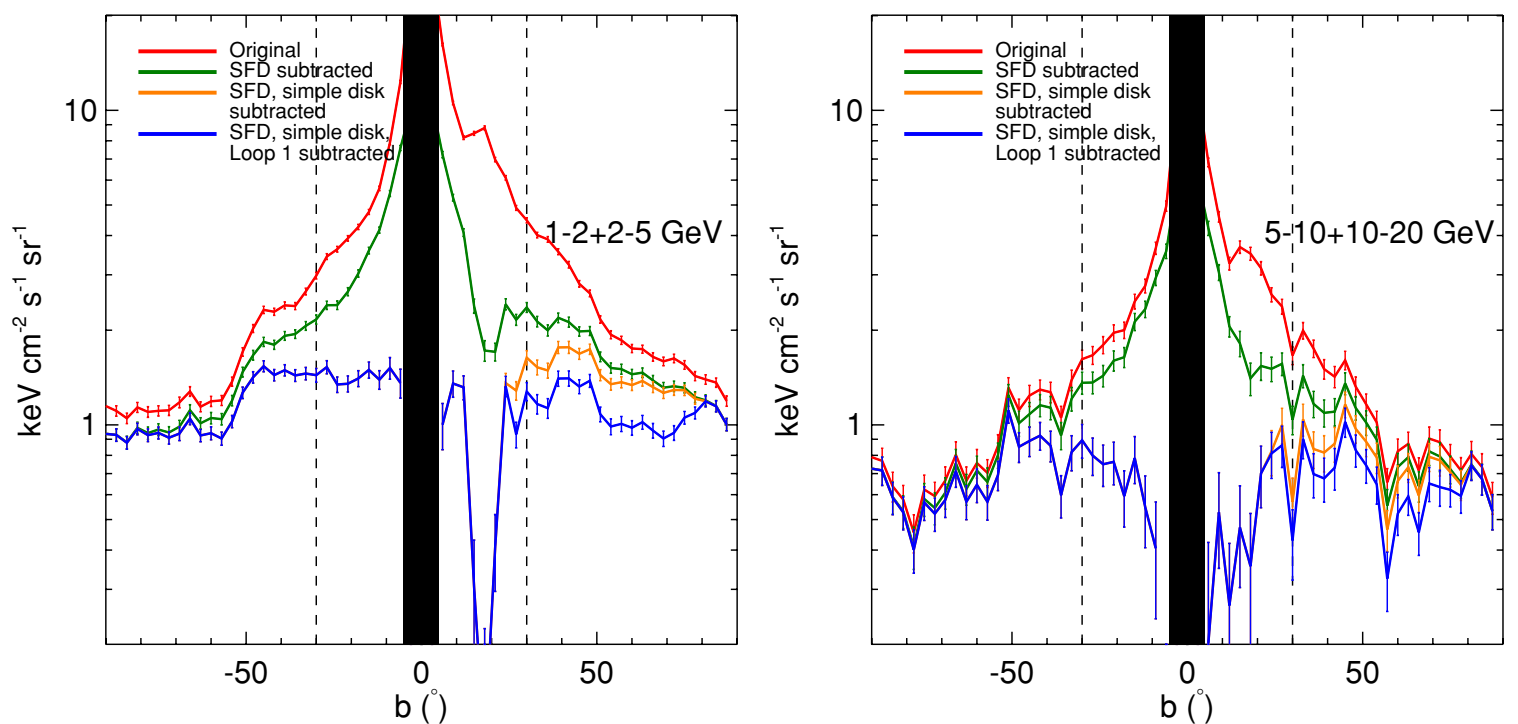

Figure 9. Intensity averaged over the central $20^{\circ}$ in longitude, as a function of latitude, for (left) the averaged 1-2 and 2-5 GeV maps, and (right) the averaged 5-10 and $10-20 \mathrm{GeV}$ maps. We construct great circle arcs perpendicular to the $l=0$ great circle, extending $10^{\circ}$ in each direction (east and west), and average the emission over each such arc; the " $b$ " label corresponding to each arc, and the $x$-axis of the plot, refers to the value of $b$ at $l=0$. Different lines show the results at different stages of the template subtraction process. The large oversubtraction at $b \sim 15^{\circ}$ in the north, especially pronounced in the low-energy data, is associated with a bright feature in the SFD dust map.

(A color version of this figure is available in the online journal.)

Table 4

Corresponding Template-fitting Coefficients and Errors in Figure 14

\begin{tabular}{lrcrrr}
\hline \hline$E$ Range $(\mathrm{GeV})$ & Energy & Uniform & \multicolumn{1}{c}{ SFD Dust } & \multicolumn{1}{c}{ Whole Bubble } & $0.5-1.0 \mathrm{GeV}-\mathrm{SFD}$ \\
\hline $0.3-0.5$ & 0.4 & $1.759 \pm 0.006$ & $0.883 \pm 0.012$ & $-0.026 \pm 0.026$ & $1.181 \pm 0.017$ \\
$0.5-0.9$ & 0.7 & $1.446 \pm 0.006$ & $0.905 \pm 0.013$ & $0.008 \pm 0.029$ & $1.275 \pm 0.018$ \\
$0.9-1.7$ & 1.3 & $1.208 \pm 0.008$ & $0.929 \pm 0.016$ & $0.258 \pm 0.034$ & $0.897 \pm 0.020$ \\
$1.7-3.0$ & 2.2 & $1.088 \pm 0.006$ & $0.679 \pm 0.012$ & $0.375 \pm 0.029$ & $0.736 \pm 0.017$ \\
$3.0-5.3$ & 4.0 & $0.921 \pm 0.007$ & $0.427 \pm 0.014$ & $0.428 \pm 0.034$ & $0.530 \pm 0.019$ \\
$5.3-9.5$ & 7.1 & $0.759 \pm 0.008$ & $0.231 \pm 0.015$ & $0.371 \pm 0.039$ & $0.400 \pm 0.021$ \\
$9.5-16.9$ & 12.7 & $0.580 \pm 0.009$ & $0.131 \pm 0.016$ & $0.269 \pm 0.043$ & $0.270 \pm 0.023$ \\
$16.9-30.0$ & 22.5 & $0.522 \pm 0.011$ & $0.072 \pm 0.016$ & $0.290 \pm 0.053$ & $0.261 \pm 0.020$ \\
$30.0-53.3$ & 40.0 & $0.565 \pm 0.014$ & $0.015 \pm 0.021$ & $0.225 \pm 0.066$ & $0.141 \pm 0.034$ \\
$53.3-94.9$ & 71.1 & $0.631 \pm 0.021$ & $0.010 \pm 0.033$ & $0.364 \pm 0.096$ & $0.065 \pm 0.053$ \\
$94.9-168.7$ & 126.5 & $0.615 \pm 0.029$ & $0.083 \pm 0.048$ & $0.337 \pm 0.127$ & $-0.032 \pm 0.076$ \\
$168.7-300.0$ & 225.0 & $0.441 \pm 0.037$ & $0.149 \pm 0.068$ & $-0.104 \pm 0.137$ & $0.089 \pm 0.097$ \\
\hline
\end{tabular}

in the intensity and spectral index between the bubble edge and interior, and the northern and southern bubbles. The resulting fitted spectra are shown in Figure 16. And the corresponding fitting coefficients and errors of each template are listed in Table 5-8, respectively. In Figure 17, we replace the simple disk model with the Haslam $408 \mathrm{MHz}$ map as the IC template, and employ the SFD map, a uniform background, the Loop I template, and the double-lobed bubble (left panel) or the north and south bubble (right panel) templates in the fitting. Our conclusion is that the Fermi bubbles appear to be north-south symmetric and spatially and spectrally uniform, with a hard spectrum. This statement is largely independent of our choice of template for the disk IC emission.

\subsection{The "Fermi Bubbles" versus the "Fermi Haze"}

Dobler et al. (2010) employed essentially the same template regression methods, claimed that the gamma-ray emission not accounted for by the known foregrounds could be well fitted by a bivariate Gaussian with $\sigma_{b}=25^{\circ}, \sigma_{l}=15^{\circ}$. With the improved $1.6 \mathrm{yr}$ data, the edges of the excess at high latitudes are seen to be quite sharp, and are not well described by a Gaussian fall-off in intensity. However, the question of whether the excess is better modeled as an "egg" or a pair of "bubbles" is more subtle.

The choice of the bivariate Gaussian template by Dobler et al. (2010) was intended to remove as much of the remaining gamma-ray signal as possible, once the $\pi^{0}$ and soft IC emission had been regressed out, minimizing large-scale residuals. The fits were performed with only $|b|<5$ masked out. In this work, on the other hand, we have masked out all emission with $|b|<30^{\circ}$; when attempting to subtract the disk-correlated emission, and delineating the "bubbles" template, our goal has been to isolate the sharp-edged features from the more slowly spatially varying emission, not to account for all the observed emission.

This difference in approach can be seen in the non-negligible residuals around the inner Galaxy $\left(|b| \lesssim 20^{\circ}\right)$ in many of our maps (for example, Figure 13). An attempt to fit all the residual emission simultaneously with a simple template may well require a template closer to that used by Dobler et al. (2010), rather than the bubble template. However, the sharp edges now visible in the data, and their alignment with the edges of the WMAP haze and ROSAT X-ray features (as we will discuss in 

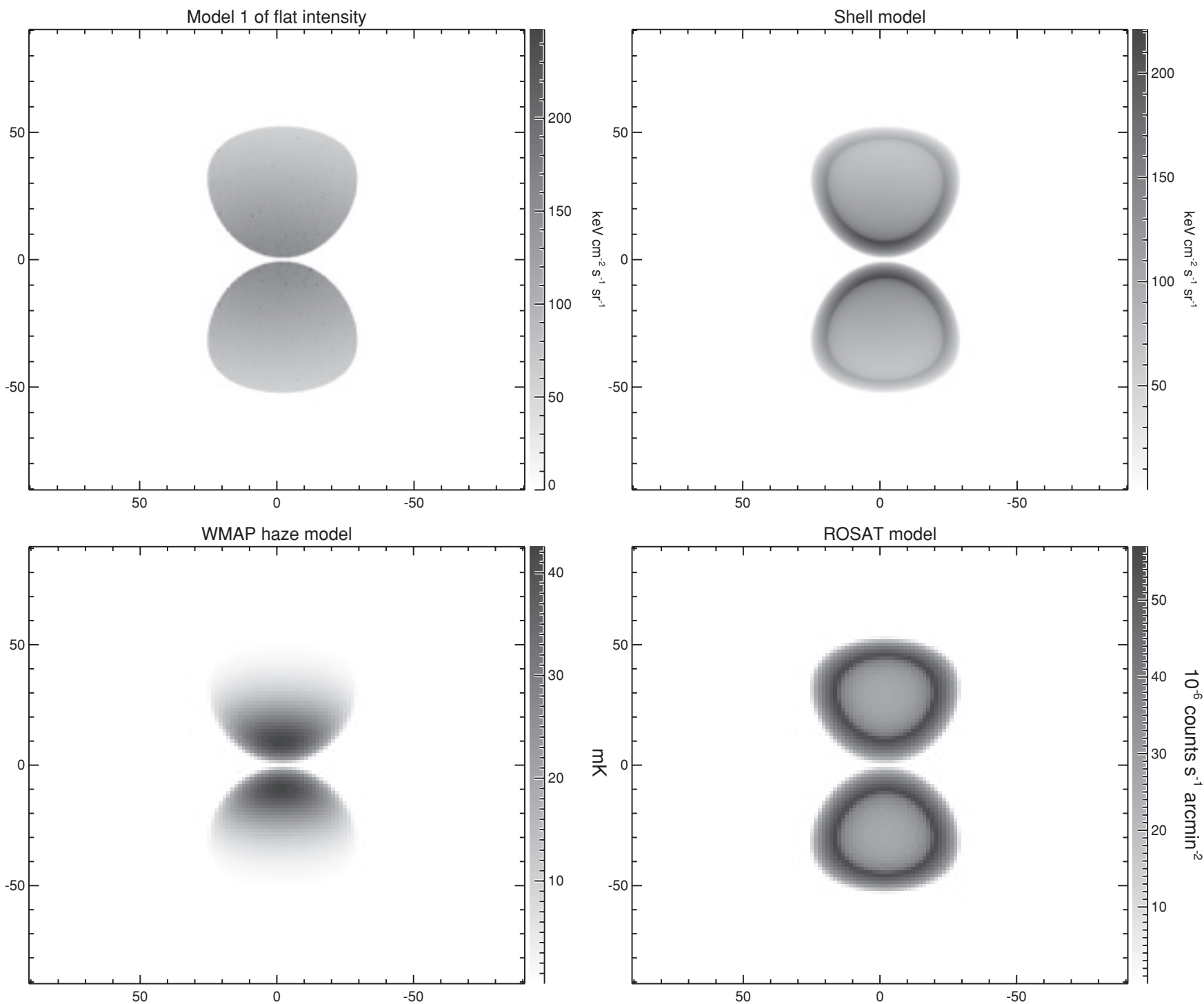

Figure 10. Projected emission from four emissivity distributions to illustrate the qualitative features of each. Top left: a toy model of the Fermi bubbles with flat projected intensity. In this model, the volume emissivity (assuming the ISM is optically thin to gamma rays) is proportional to $\left(R^{2}-r^{2}\right)^{-1 / 2}$ for $r<R$, and zero otherwise, where $R=3.5 \mathrm{kpc}$ is the approximate radius of the two bubbles, and $r$ is the distance to the center of the north or south bubble. Top right: a bubble model with compressed gas shells with a thickness of $0.5 \mathrm{kpc}$; the electron CR density in the shell is a factor of 5 higher than in the interior of the bubbles. For this model, a limb-brightened edge of the bubbles is clearly visible, a feature which is not seen in the Fermi data. Bottom left: an illustrative toy model for the WMAP haze. The haze synchrotron emissivity depends on the electron CR density and the magnetic field; here we take $B=B_{0} e^{-z / z_{0}}$, where $z_{0}=2 \mathrm{kpc}$, and show the line-of-sight integral of this B-field through the bubble volume. Even though the synchrotron emissivity is not simply the product of CR density times field strength, this panel suggests that the decreasing intensity of the WMAP haze at high latitudes is due to the decay of the Galactic magnetic field away from the Galactic plane. Bottom right: a toy model for the ROSAT X-ray features. The observed soft X-rays are limb brightened and we assume all gas is uniformly distributed within a compressed shell, with no contribution from the interior, and X-ray emission is proportional to the gas density squared. The thickness of the shell is $1 \mathrm{kpc}$.

Table 5

Corresponding Template Fitting Coefficients and Errors in the Upper Left Panel of Figure 16

\begin{tabular}{lrccccrr}
\hline \hline$E$ Range $(\mathrm{GeV})$ & Energy & Uniform & \multicolumn{1}{c}{ SFD Dust } & \multicolumn{1}{c}{ Simple Disk } & Inner Bubble & Outer Bubble & Simple Loop I \\
\hline $0.3-0.5$ & 0.4 & $1.681 \pm 0.006$ & $1.201 \pm 0.011$ & $0.683 \pm 0.027$ & $0.071 \pm 0.042$ & $0.004 \pm 0.040$ & $0.490 \pm 0.015$ \\
$0.5-0.9$ & 0.7 & $1.365 \pm 0.007$ & $1.279 \pm 0.012$ & $0.607 \pm 0.030$ & $0.215 \pm 0.048$ & $0.207 \pm 0.045$ & $0.475 \pm 0.016$ \\
$0.9-1.7$ & 1.3 & $1.142 \pm 0.008$ & $1.179 \pm 0.014$ & $0.498 \pm 0.036$ & $0.354 \pm 0.057$ & $0.293 \pm 0.053$ & $0.407 \pm 0.019$ \\
$1.7-3.0$ & 2.2 & $1.034 \pm 0.006$ & $0.876 \pm 0.011$ & $0.403 \pm 0.029$ & $0.370 \pm 0.047$ & $0.491 \pm 0.045$ & $0.373 \pm 0.016$ \\
$3.0-5.3$ & 4.0 & $0.880 \pm 0.008$ & $0.554 \pm 0.013$ & $0.426 \pm 0.035$ & $0.307 \pm 0.056$ & $0.393 \pm 0.054$ & $0.247 \pm 0.018$ \\
$5.3-9.5$ & 7.1 & $0.731 \pm 0.009$ & $0.322 \pm 0.014$ & $0.284 \pm 0.039$ & $0.330 \pm 0.064$ & $0.354 \pm 0.061$ & $0.207 \pm 0.021$ \\
$9.5-16.9$ & 12.7 & $0.562 \pm 0.010$ & $0.193 \pm 0.015$ & $0.265 \pm 0.044$ & $0.100 \pm 0.070$ & $0.293 \pm 0.069$ & $0.087 \pm 0.023$ \\
$16.9-30.0$ & 22.5 & $0.506 \pm 0.012$ & $0.128 \pm 0.018$ & $0.201 \pm 0.054$ & $0.182 \pm 0.088$ & $0.335 \pm 0.087$ & $0.121 \pm 0.029$ \\
$30.0-53.3$ & 40.0 & $0.556 \pm 0.015$ & $0.041 \pm 0.021$ & $0.098 \pm 0.065$ & $0.190 \pm 0.106$ & $0.243 \pm 0.107$ & $0.087 \pm 0.036$ \\
$53.3-94.9$ & 71.1 & $0.627 \pm 0.022$ & $0.020 \pm 0.030$ & $0.187 \pm 0.093$ & $0.206 \pm 0.151$ & $0.294 \pm 0.153$ & $-0.045 \pm 0.048$ \\
$94.9-168.7$ & 126.5 & $0.620 \pm 0.030$ & $0.081 \pm 0.043$ & $0.037 \pm 0.129$ & $0.180 \pm 0.209$ & $0.431 \pm 0.207$ & $-0.098 \pm 0.064$ \\
$168.7-300.0$ & 225.0 & $0.435 \pm 0.038$ & $0.179 \pm 0.062$ & $-0.065 \pm 0.155$ & $0.145 \pm 0.242$ & $-0.178 \pm 0.206$ & $0.097 \pm 0.086$
\end{tabular}



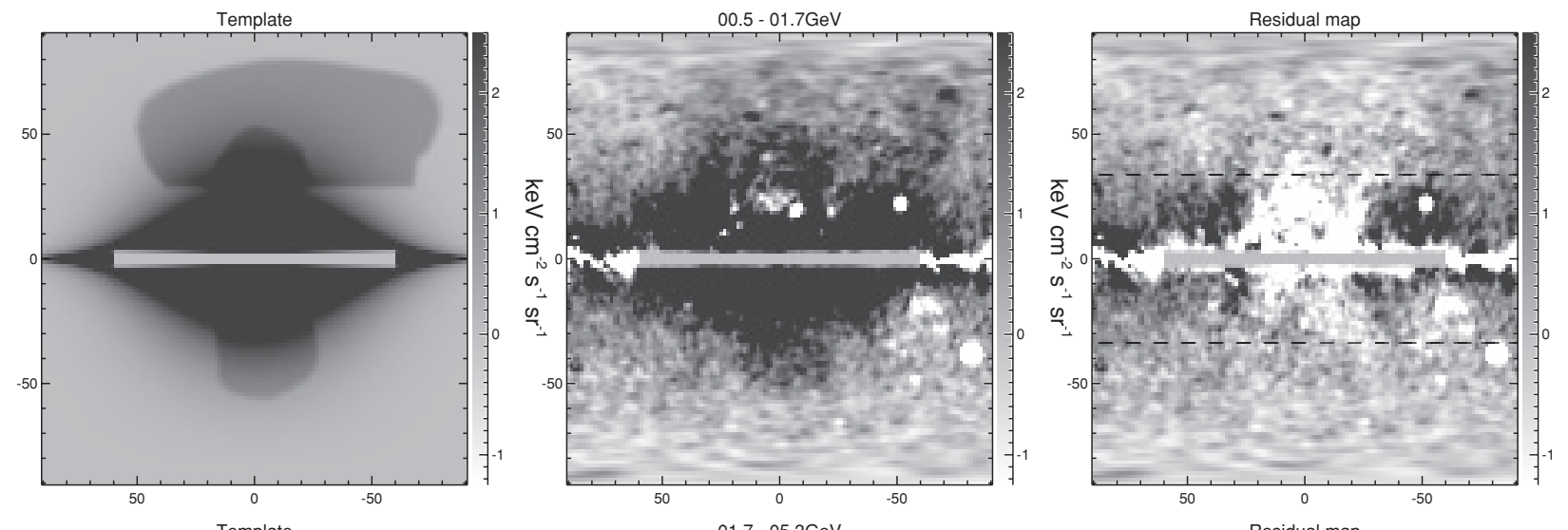

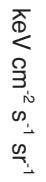
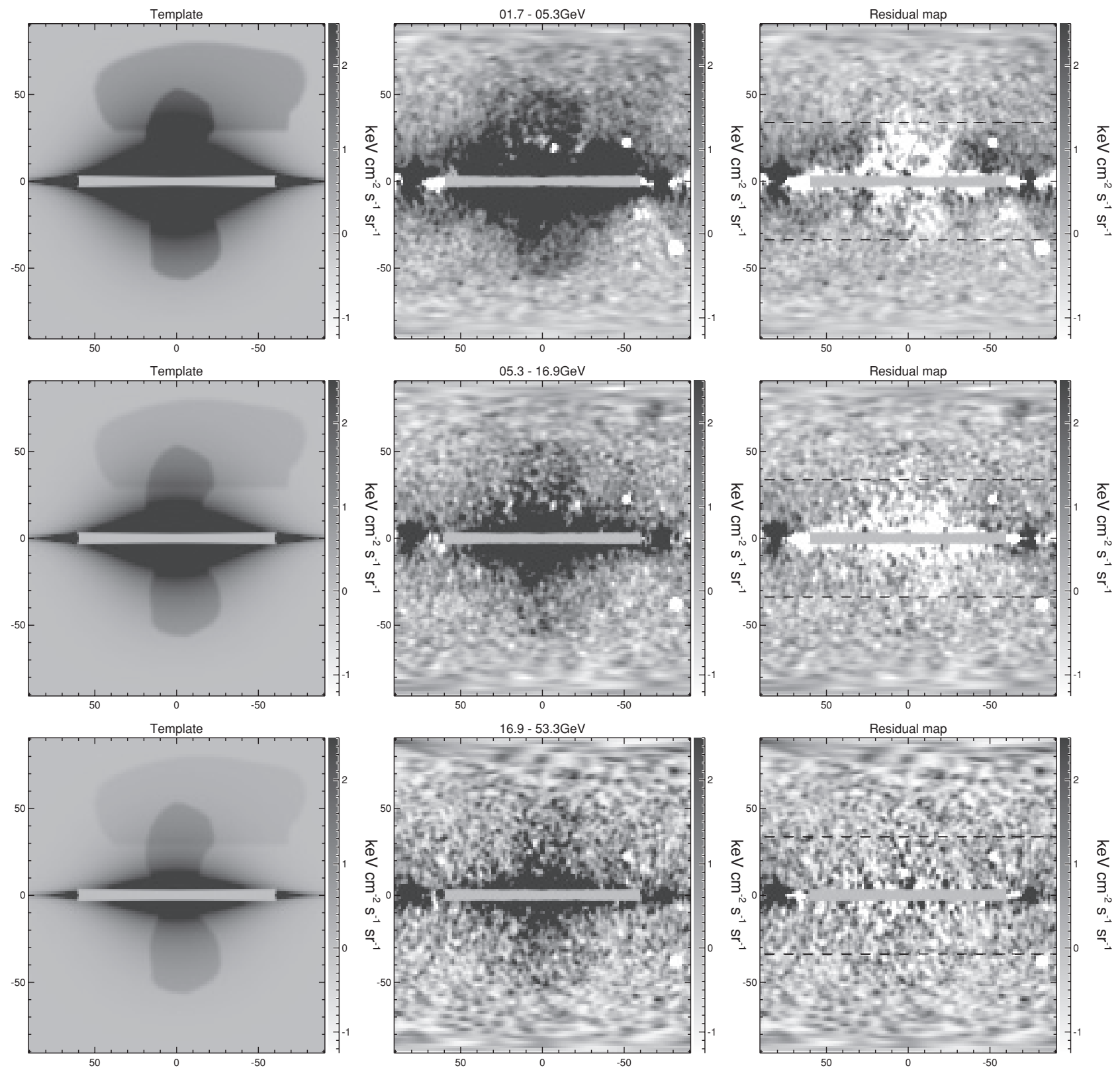

Figure 11. Models obtained from the multi-template fits, compared with the Fermi maps, in different energy bins. The left column shows the linear combination of the disk, Loop I, uniform, and bubble templates that provide the best fit to the Fermi maps after subtracting the best-fit SFD dust template (shown in the middle column). The difference maps between the combined template and the data are shown in the right column. The template fitting is done for the region with $|b|>30^{\circ}$ to avoid contaminations from the Galactic disk (shown with black dashed line in the right column residual maps). The subtraction of the model largely removes the features seen in the Fermi maps with $|b|>30^{\circ}$. We use the same gray scale for all the panels. We find that both the disk IC template and Loop I features fade off with increasing energy, but the bubble template is required for all the energy bands and does not fade off with increasing energy. The oversubtraction in the residual maps, especially at lower energy bins, is due to the simple disk IC model, which is not a good template across the entire disk. However, outside the masked region, the residual maps are consistent with Poisson noise without obvious large-scale features. 
Table 6

Corresponding Template Fitting Coefficients and Errors in the Upper Right Panel of Figure 16

\begin{tabular}{lrcccrcr}
\hline \hline$E$ Range $(\mathrm{GeV})$ & Energy & Uniform & \multicolumn{1}{c}{ SFD Dust } & \multicolumn{1}{c}{ Simple Disk } & \multicolumn{1}{c}{ North Bubble } & \multicolumn{1}{c}{ South Bubble } & Simple Loop I \\
\hline $0.3-0.5$ & 0.4 & $1.679 \pm 0.006$ & $1.205 \pm 0.011$ & $0.683 \pm 0.027$ & $-0.042 \pm 0.050$ & $0.069 \pm 0.037$ & $0.496 \pm 0.015$ \\
$0.5-0.9$ & 0.7 & $1.363 \pm 0.007$ & $1.282 \pm 0.012$ & $0.603 \pm 0.030$ & $0.140 \pm 0.056$ & $0.241 \pm 0.041$ & $0.483 \pm 0.017$ \\
$0.9-1.7$ & 1.3 & $1.138 \pm 0.008$ & $1.187 \pm 0.015$ & $0.493 \pm 0.035$ & $0.171 \pm 0.065$ & $0.385 \pm 0.049$ & $0.421 \pm 0.020$ \\
$1.7-3.0$ & 2.2 & $1.030 \pm 0.007$ & $0.883 \pm 0.012$ & $0.384 \pm 0.029$ & $0.288 \pm 0.054$ & $0.503 \pm 0.041$ & $0.391 \pm 0.016$ \\
$3.0-5.3$ & 4.0 & $0.881 \pm 0.008$ & $0.553 \pm 0.013$ & $0.421 \pm 0.035$ & $0.368 \pm 0.064$ & $0.346 \pm 0.049$ & $0.247 \pm 0.019$ \\
$5.3-9.5$ & 7.1 & $0.731 \pm 0.009$ & $0.321 \pm 0.014$ & $0.283 \pm 0.039$ & $0.354 \pm 0.072$ & $0.337 \pm 0.055$ & $0.207 \pm 0.022$ \\
$9.5-16.9$ & 12.7 & $0.562 \pm 0.010$ & $0.195 \pm 0.016$ & $0.248 \pm 0.044$ & $0.157 \pm 0.078$ & $0.230 \pm 0.063$ & $0.097 \pm 0.024$ \\
$16.9-30.0$ & 22.5 & $0.509 \pm 0.012$ & $0.124 \pm 0.018$ & $0.196 \pm 0.053$ & $0.353 \pm 0.100$ & $0.216 \pm 0.076$ & $0.116 \pm 0.030$ \\
$30.0-53.3$ & 40.0 & $0.556 \pm 0.015$ & $0.042 \pm 0.021$ & $0.094 \pm 0.064$ & $0.190 \pm 0.117$ & $0.232 \pm 0.097$ & $0.092 \pm 0.038$ \\
$53.3-94.9$ & 71.1 & $0.628 \pm 0.022$ & $0.020 \pm 0.030$ & $0.184 \pm 0.093$ & $0.264 \pm 0.164$ & $0.243 \pm 0.140$ & $-0.045 \pm 0.049$ \\
$94.9-168.7$ & 126.5 & $0.614 \pm 0.030$ & $0.091 \pm 0.044$ & $0.004 \pm 0.126$ & $0.110 \pm 0.208$ & $0.450 \pm 0.195$ & $-0.068 \pm 0.067$ \\
$168.7-300.0$ & 225.0 & $0.429 \pm 0.039$ & $0.186 \pm 0.064$ & $-0.058 \pm 0.160$ & $-0.169 \pm 0.254$ & $0.061 \pm 0.222$ & $0.107 \pm 0.092$ \\
\hline
\end{tabular}

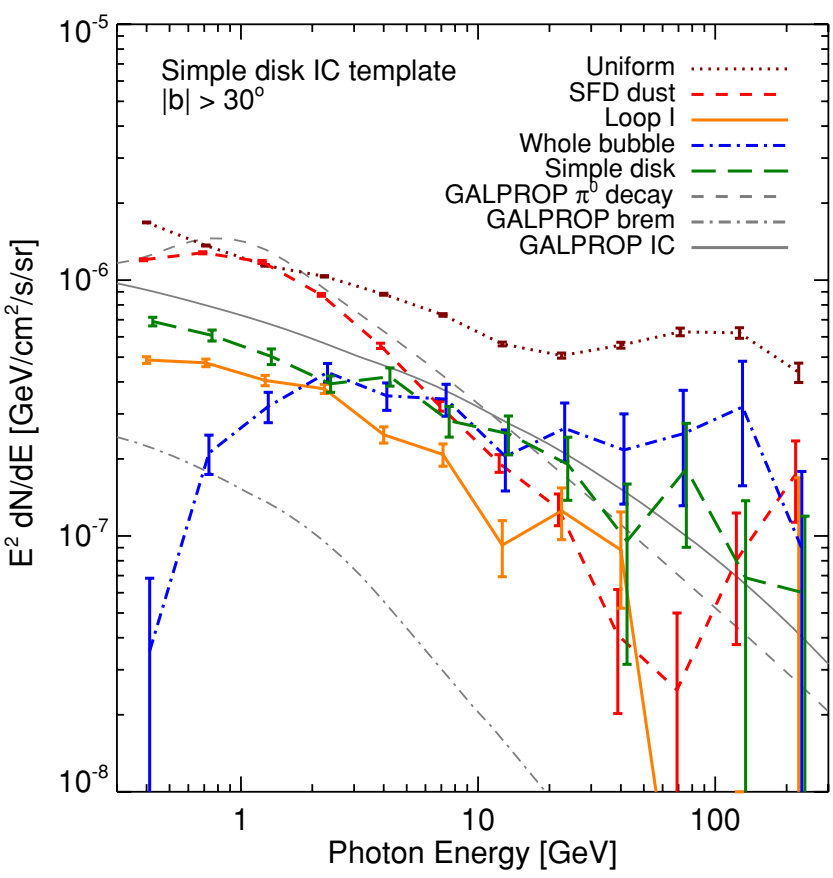

Figure 12. Correlation spectra for the 5-template fit employing a simple disk model for the IC (and to a lesser degree bremsstrahlung) emission from SNshock-accelerated electrons (see Section 3.2.2). The SFD-correlated spectrum is shown by the red short-dashed line which roughly traces $\pi^{0}$ emission (the gray dashed line indicates a GALPROP prediction for $\pi^{0}$ emission). The diskcorrelated emission is shown by the green dashed line, which traces the soft IC (gray triple-dot-dashed line) and bremsstrahlung (gray dot-dashed line) component. The spectrum of the uniform emission, which traces the isotropic background (including possible CR contamination), is shown as a dotted brown line. The solid orange line indicates the spectrum of emission correlated with Loop I, which has a similar spectrum to the disk-correlated emission. Finally, the blue dot-dashed line shows the spectrum correlated with the Fermi bubble template. The bubble component has a notably harder (consistent with flat) spectrum than the other template-correlated spectra, and the models for the various emission mechanism generated from GALPROP, indicating that the Fermi bubbles constitute a distinct component with a hard spectrum. The fitting is done over the $|b|>30^{\circ}$ region. Note that these GALPROP "predictions" are intended only to indicate the expected spectral shape for these emission components, for reference. The correlation coefficients for the SFD map and simple disk model are multiplied by the average value of these maps in the bubble region (defined by the bottom right panel of Figure 3 , with a $|b|>30^{\circ}$ cut) to obtain the associated gamma-ray emission; see Section 3.2.2 for details and Table 2 for a summary of the normalization factors.

(A color version of this figure is available in the online journal.)

Section 4), motivate us to consider the bubbles as originating from a distinct physical mechanism. While the bubbles probably do not constitute the entire "Fermi haze" discussed by Dobler et al. (2010), they are certainly a major component of it, dominating the signal at high latitudes. The question of whether the remaining residual gamma rays represent a separate physical mechanism is an interesting one that we defer to future work.

\section{THE FERMI BUBBLES SEEN IN OTHER MAPS}

In this section, we compare the $1-5 \mathrm{GeV}$ Fermi bubble with the ROSAT $1.5 \mathrm{keV}$ soft X-ray map, the WMAP $23 \mathrm{GHz}$ microwave haze, and the Haslam $408 \mathrm{MHz}$ map. The striking similarities of several morphological features in these maps strongly suggest a common physical origin for the Fermi bubbles, WMAP haze, and X-ray edges toward the GC (Figure 18).

\subsection{Comparison with ROSAT X-ray Features}

The ROSAT all-sky survey provides full-sky images with FWHM $12^{\prime}$ at energies from $0.5-2 \mathrm{keV} .^{13}$ We compare the morphology of the X-ray features in ROSAT $1.5 \mathrm{keV}$ map with the edges of the Fermi bubbles in detail in Figure 19. The limb brightened X-ray features align with the edges of both the north and south Fermi bubbles. Hints of the whole north bubble are also visible in ROSAT, as well as two sharp edges in the south that trace the south Fermi bubble close to the disk. We show the ROSAT $1.5 \mathrm{keV}$ map overplotted with the edges of the Fermi bubbles, the northern arc, the "donut" and the Loop I features in the right panels of Figure 19. The appearance of the X-ray edges in the ROSAT $1.5 \mathrm{keV}$ map, coincident with the Fermi bubble edges, strongly supports the physical reality of these sharp edges.

In Figure 20, we subtract the ROSAT $1.0 \mathrm{keV}$ soft X-ray map from the $1.5 \mathrm{keV}$ map to clean up the foreground. We find that the extended Loop I feature has a softer spectrum than the $\mathrm{X}$-ray features associated with the bubble edges, and is largely removed in the difference map (lower left panel of Figure 20). The residual features strikingly overlap with the edges of the Fermi bubbles (lower right panel). No other noticeable largescale features appear in the residual X-ray map which do not appear in the gamma rays.

\subsection{Comparison with WMAP Microwave Haze}

The WMAP haze is the residual remaining in WMAP microwave data after regressing out contributions from thermal dust, free-free, and "soft synchrotron" traced by the Haslam

\footnotetext{
13 http://hea-www.harvard.edu/rosat/rsdc.html
} 

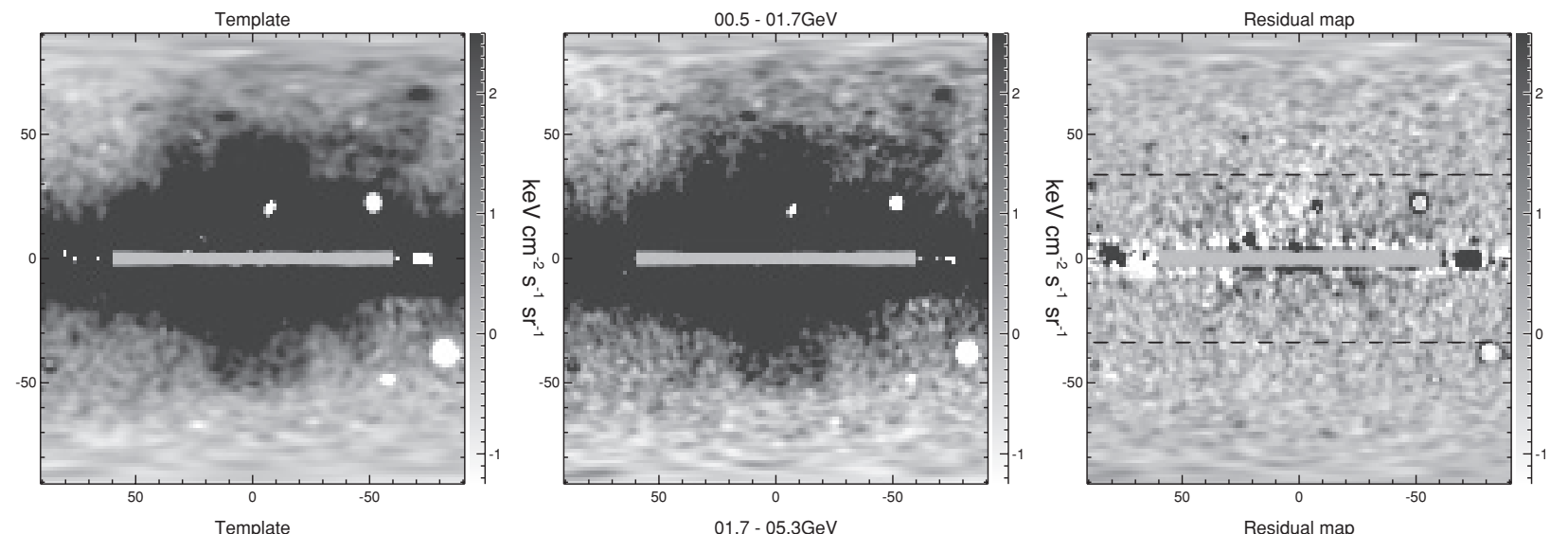

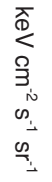
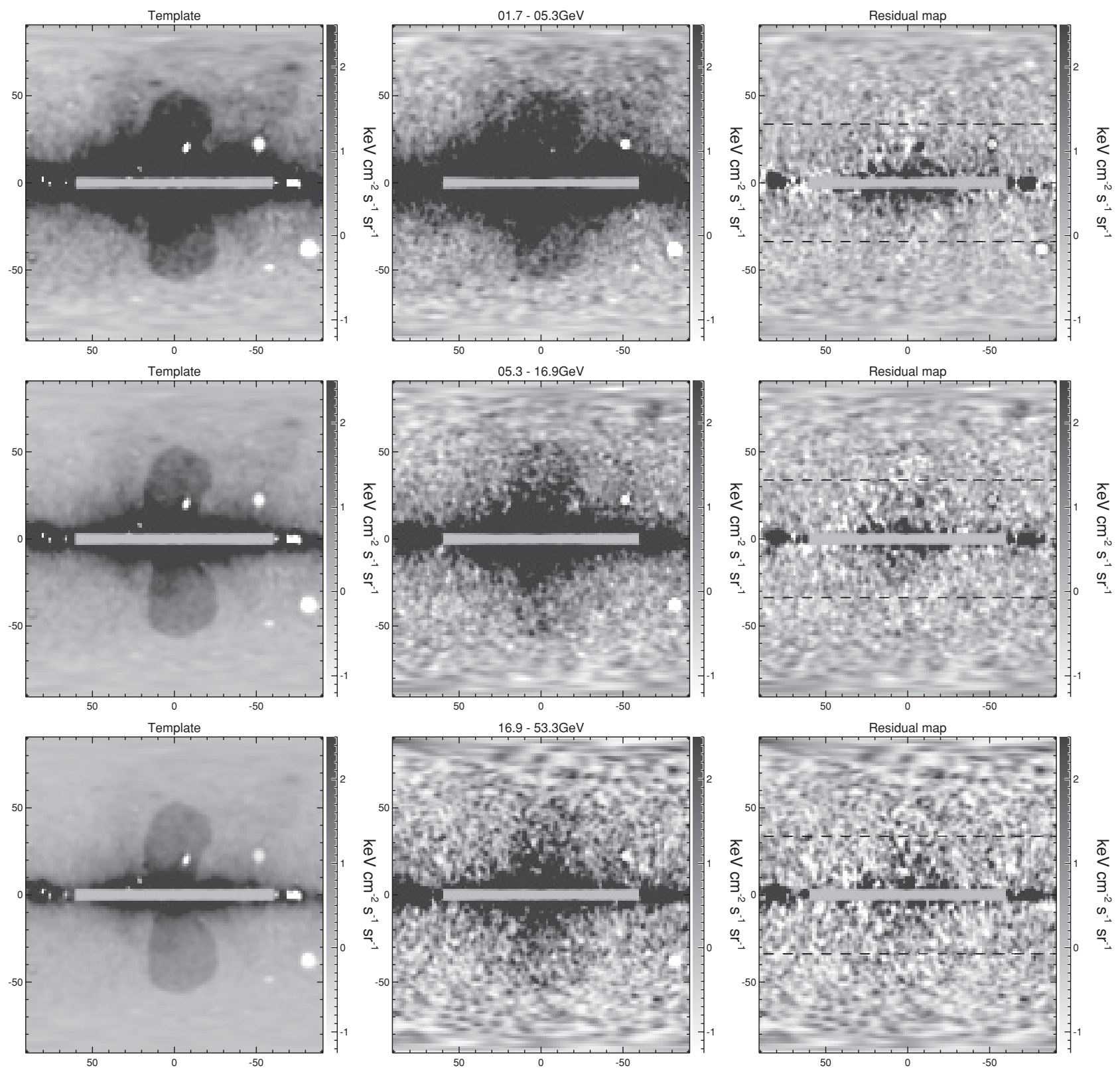

Figure 13. Same as Figure 11, but using the Fermi $0.5-1 \mathrm{GeV}$ residual map after subtracting emission correlated with the SFD dust map (to remove the $\pi^{0}$ gammas) as a template for low-latitude IC emission (originating primarily from scatterings on starlight, and likely involving softer SN-shock-accelerated electrons). The left column shows the best-fit combined template including the residual 0.5-1 GeV map, uniform, SFD dust, and bubble templates. The middle column shows the Fermi-LAT data in different energy bins. The difference maps between the combined template and the data are shown in the right column. The template fitting is done over the region with $|b|>30^{\circ}$ (shown with black dashed line in the right column residual maps). We use the same gray scale for all the panels. We find that the bubble template does not fade away with increasing energy. The 4-template fit works extremely well, at $|b|>30^{\circ}$ the residual maps are consistent with Poisson noise without obvious large-scale features, and there are no obvious sharp features in the data closer to the disk. 
Table 7

Corresponding Template Fitting Coefficients and Errors in the Lower Left Panel of Figure 16

\begin{tabular}{lrcccrr}
\hline \hline$E$ Range $(\mathrm{GeV})$ & Energy & Uniform & SFD Dust & Inner Bubble & Outer Bubble & $0.5-1.0 \mathrm{GeV}-\mathrm{SFD}$ \\
\hline $0.3-0.5$ & 0.4 & $1.759 \pm 0.006$ & $0.883 \pm 0.012$ & $0.011 \pm 0.035$ & $-0.069 \pm 0.037$ & $1.180 \pm 0.017$ \\
$0.5-0.9$ & 0.7 & $1.446 \pm 0.006$ & $0.905 \pm 0.013$ & $0.005 \pm 0.039$ & $0.012 \pm 0.041$ & $1.275 \pm 0.018$ \\
$0.9-1.7$ & 1.3 & $1.208 \pm 0.008$ & $0.929 \pm 0.016$ & $0.284 \pm 0.047$ & $0.230 \pm 0.048$ & $0.896 \pm 0.020$ \\
$1.7-3.0$ & 2.2 & $1.088 \pm 0.006$ & $0.680 \pm 0.012$ & $0.309 \pm 0.039$ & $0.444 \pm 0.041$ & $0.737 \pm 0.017$ \\
$3.0-5.3$ & 4.0 & $0.921 \pm 0.007$ & $0.427 \pm 0.014$ & $0.399 \pm 0.047$ & $0.459 \pm 0.049$ & $0.530 \pm 0.019$ \\
$5.3-9.5$ & 7.1 & $0.759 \pm 0.008$ & $0.231 \pm 0.015$ & $0.362 \pm 0.053$ & $0.382 \pm 0.056$ & $0.400 \pm 0.021$ \\
$9.5-16.9$ & 12.7 & $0.580 \pm 0.009$ & $0.131 \pm 0.016$ & $0.195 \pm 0.057$ & $0.351 \pm 0.063$ & $0.271 \pm 0.023$ \\
$16.9-30.0$ & 22.5 & $0.523 \pm 0.011$ & $0.069 \pm 0.015$ & $0.218 \pm 0.071$ & $0.368 \pm 0.079$ & $0.261 \pm 0.018$ \\
$30.0-53.3$ & 40.0 & $0.565 \pm 0.012$ & $0.014 \pm 0.015$ & $0.202 \pm 0.088$ & $0.249 \pm 0.096$ & $0.144 \pm 0.025$ \\
$53.3-94.9$ & 71.1 & $0.631 \pm 0.021$ & $0.010 \pm 0.033$ & $0.337 \pm 0.128$ & $0.394 \pm 0.140$ & $0.065 \pm 0.053$ \\
$94.9-168.7$ & 126.5 & $0.614 \pm 0.029$ & $0.084 \pm 0.048$ & $0.232 \pm 0.164$ & $0.455 \pm 0.190$ & $-0.029 \pm 0.076$ \\
$168.7-300.0$ & 225.0 & $0.440 \pm 0.037$ & $0.152 \pm 0.069$ & $0.021 \pm 0.195$ & $-0.262 \pm 0.175$ & $0.088 \pm 0.096$ \\
\hline
\end{tabular}

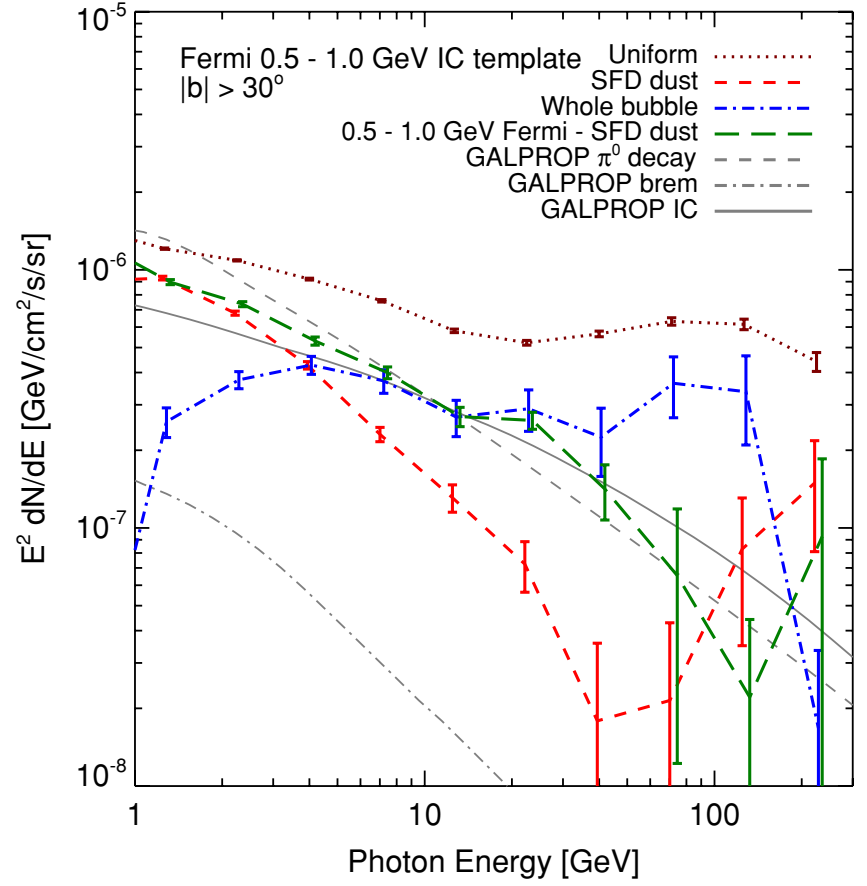

Figure 14. Same as Figure 12, but correlation spectra for the 4-template fit employing the Fermi $0.5-1 \mathrm{GeV}$ residual map (after subtracting the SFD dust) as a template for the starlight IC. The line style is the same as Figure 12. Again, we find that the spectrum correlated with the Fermi bubble template (blue dot-dashed line) is harder (consistent with flat in $E^{2} d N / d E$ ) than the spectra correlated with the other templates, and the models for the various emission mechanisms generated from GALPROP, indicating that the Fermi bubbles constitute a distinct gamma-ray component with a hard spectrum. The fitting is done for $|b|>30^{\circ}$. As in Figure 12, the correlation spectra have been normalized to a reference region; see Section 3.2.2 for details, and Table 2 for a summary of the normalization factors.

(A color version of this figure is available in the online journal.)

$408 \mathrm{MHz}$ radio survey (Haslam et al. 1982). Therefore, it is by construction harder than the Haslam-correlated emission. We will show in this section that the WMAP synchrotron haze appears to be associated with the Fermi bubbles.

Figure 21 shows a detailed morphological comparison of the south Fermi bubble at $1-5 \mathrm{GeV}$ with the southern part of the WMAP microwave haze at $23 \mathrm{GHz}$ ( $K$ band). The edge of the Fermi bubbles, marked in green dashed line in the top right and lower right panels, closely traces the edge of the WMAP haze. The smaller latitudinal extension of the WMAP haze may be due to the decay of the magnetic field strength with latitude.
These striking morphological similarities between the WMAP microwave haze and Fermi gamma-ray bubble can be readily explained if the same electron $\mathrm{CR}$ population is responsible for both excesses, with the electron CRs interacting with the galactic magnetic field to produce synchrotron, and interacting with the ISRF to produce IC emission.

In Figure 22, we show the difference maps between the $23 \mathrm{GHz} W M A P$ haze and the $33 \mathrm{GHz}$ and $41 \mathrm{GHz}$ haze maps. The difference maps contain no apparent features and indicate a common spectrum of different regions inside the WMAP haze, suggesting a single physical origin for the bulk of the signal. We have reached the same conclusion for the Fermi bubbles in Figure 7.

Besides the similarity of the morphology, the relatively hard spectrum of the Fermi bubble also motivates a common physical origin with the WMAP haze. We now provide a simple estimate of the microwave synchrotron and gamma-ray ICS signals from one single population of hard electrons distributed in the inner Galaxy, to demonstrate that the magnitudes and spectral indices of the two signals are consistent for reasonable parameter values.

For a highly relativistic electron scattering on low-energy photons, the spectrum of upscattered photons is given by Blumenthal \& Gould (1970) (Cholis et al. 2009a),

$$
\begin{aligned}
\frac{d N}{d E_{\gamma} d \epsilon d t}= & \frac{3}{4} \sigma_{T} c \frac{\left(m_{e} c^{2}\right)^{2}}{\epsilon E_{e}^{2}}(2 q \log q+(1+2 q)(1-q) \\
& \left.+0.5(1-q)(\Gamma q)^{2} /(1+\Gamma q)\right) n(\epsilon) \\
\Gamma= & 4 \epsilon E_{e} /\left(m_{e} c^{2}\right)^{2}, \quad q=\frac{E_{\gamma}}{E_{e}} \frac{1}{\Gamma\left(1-E_{\gamma} / E_{e}\right)}, \\
& \epsilon<E_{\gamma}<E_{e} \Gamma /(1+\Gamma) .
\end{aligned}
$$

Here $\epsilon$ is the initial photon energy, $E_{e}$ is the electron energy, $E_{\gamma}$ is the energy of the upscattered gamma ray, and $n(\epsilon)$ describes the energy distribution of the soft photons per unit volume. Where $\Gamma \ll 1$, in the Thomson limit, the average energy of the upscattered photons is given by

$$
\left\langle E_{\gamma}\right\rangle=(4 / 3) \gamma^{2}\langle\epsilon\rangle
$$

where $\gamma=E_{e} / m_{e} c^{2}$ is the Lorentz boost of the electron. In the Klein-Nishina $(\mathrm{KN})$ limit, $\Gamma \gg 1$, the spectrum instead peaks at the high-energy end, and the upscattered photon carries away almost all the energy of the electron.

Given a power-law steady-state electron spectrum with spectral index $\gamma$, the spectral index of the IC scattered gamma rays is $(\gamma+1) / 2$ in the Thomson limit, and $\gamma+1$ in the extreme $\mathrm{KN}$ 

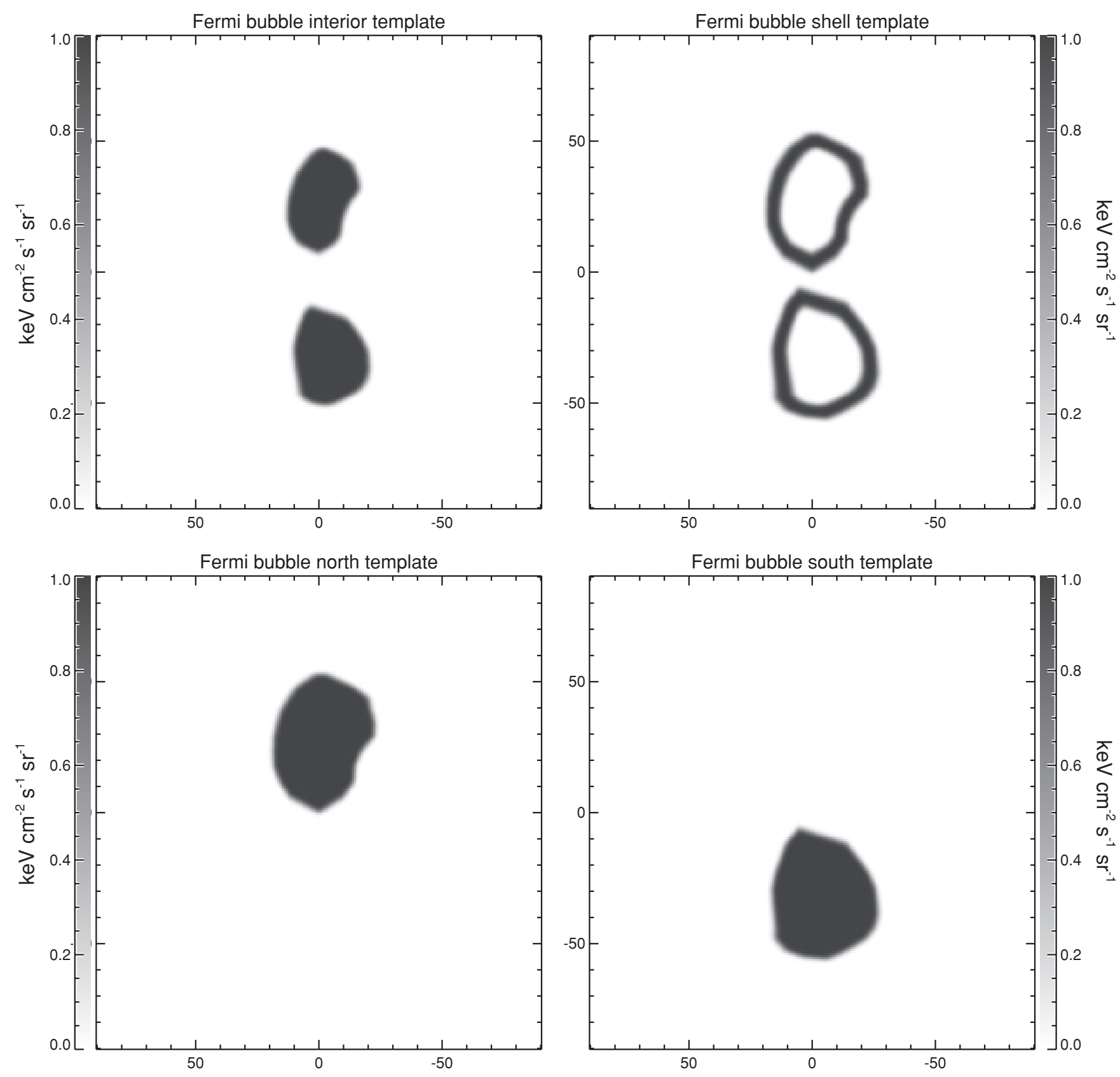

Figure 15. Top row: we split our Fermi bubble template (shown in the bottom right panel of Figure 3) into two components for template fitting: an interior template (top left) and a shell template (top right) with uniform intensity, in order to reveal any potential spectrum difference with the template fitting technique. Bottom row: we split the Fermi bubble template into north bubble template (bottom left) and south bubble template (bottom right). If the two bubbles have the same origin, they should not only have similar morphologies but also consistent spectra.

Table 8

Corresponding Template Fitting Coefficients and Errors in the Lower Right Panel of Figure 16

\begin{tabular}{lrcccrr}
\hline \hline$E$ Range $(\mathrm{GeV})$ & Energy & Uniform & \multicolumn{1}{c}{ SFD Dust } & \multicolumn{1}{c}{ North Bubble } & \multicolumn{1}{c}{ South Bubble } & $0.5-1.0 \mathrm{GeV}-\mathrm{SFD}$ \\
\hline $0.3-0.5$ & 0.4 & $1.759 \pm 0.006$ & $0.882 \pm 0.012$ & $-0.011 \pm 0.047$ & $-0.031 \pm 0.029$ & $1.180 \pm 0.017$ \\
$0.5-0.9$ & 0.7 & $1.446 \pm 0.006$ & $0.905 \pm 0.013$ & $-0.009 \pm 0.052$ & $0.014 \pm 0.032$ & $1.276 \pm 0.018$ \\
$0.9-1.7$ & 1.3 & $1.207 \pm 0.008$ & $0.931 \pm 0.016$ & $0.201 \pm 0.061$ & $0.277 \pm 0.038$ & $0.899 \pm 0.020$ \\
$1.7-3.0$ & 2.2 & $1.087 \pm 0.006$ & $0.681 \pm 0.013$ & $0.331 \pm 0.050$ & $0.391 \pm 0.033$ & $0.738 \pm 0.017$ \\
$3.0-5.3$ & 4.0 & $0.922 \pm 0.007$ & $0.424 \pm 0.014$ & $0.500 \pm 0.060$ & $0.399 \pm 0.039$ & $0.527 \pm 0.019$ \\
$5.3-9.5$ & 7.1 & $0.760 \pm 0.008$ & $0.228 \pm 0.015$ & $0.440 \pm 0.067$ & $0.342 \pm 0.045$ & $0.397 \pm 0.021$ \\
$9.5-16.9$ & 12.7 & $0.580 \pm 0.009$ & $0.132 \pm 0.016$ & $0.231 \pm 0.072$ & $0.286 \pm 0.051$ & $0.272 \pm 0.023$ \\
$16.9-30.0$ & 22.5 & $0.524 \pm 0.011$ & $0.068 \pm 0.015$ & $0.406 \pm 0.091$ & $0.236 \pm 0.060$ & $0.257 \pm 0.008$ \\
$30.0-53.3$ & 40.0 & $0.565 \pm 0.014$ & $0.014 \pm 0.015$ & $0.227 \pm 0.105$ & $0.222 \pm 0.077$ & $0.143 \pm 0.015$ \\
$53.3-94.9$ & 71.1 & $0.631 \pm 0.021$ & $0.011 \pm 0.033$ & $0.339 \pm 0.151$ & $0.376 \pm 0.115$ & $0.067 \pm 0.054$ \\
$94.9-168.7$ & 126.5 & $0.608 \pm 0.029$ & $0.091 \pm 0.049$ & $0.067 \pm 0.189$ & $0.470 \pm 0.162$ & $-0.013 \pm 0.077$ \\
$168.7-300.0$ & 225.0 & $0.437 \pm 0.038$ & $0.156 \pm 0.070$ & $-0.216 \pm 0.229$ & $-0.066 \pm 0.160$ & $0.095 \pm 0.098$ \\
\hline
\end{tabular}



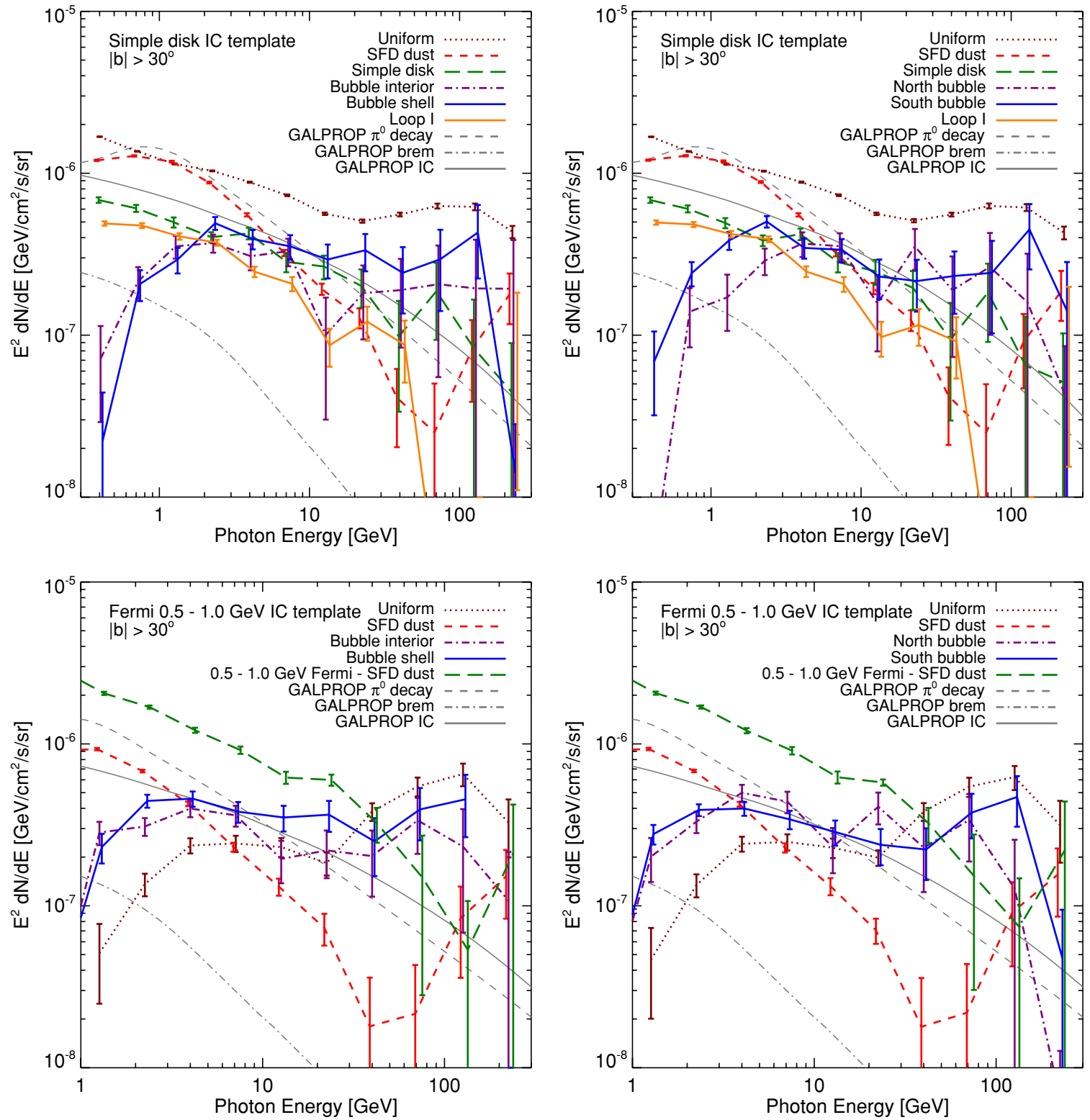

Figure 16. Same as Figures 12 and 14, but splitting the Fermi bubble template into two components for template fitting. The line styles are the same as Figure 12 . Top row: using the simple disk model as the IC template. In the left panel, we split the previous bubble template into bubble interior and bubble shell templates (see Figure 15 for the templates). The correlation coefficients of the 6-template fit involving the two bubble templates are shown. The purple dash-dotted line and blue triple-dot-dashed line are for the inner bubble and the outer shell template, respectively. The two templates have a consistent spectrum which is significantly harder than the other templates, indicating that the bubble interior and the bubble shell have the same distinct physical origin. In the right panel, we split the bubble template into north and south bubbles. As we include the Loop I template (which has a softer spectrum) in the north sky for regression fitting, the north bubble has a slightly harder spectrum than the south bubble. Again, both of the templates have harder spectra than any other components in the fit. Bottom row: employing the Fermi 0.5-1 GeV residual map (after subtracting the SFD dust) as a template for the starlight IC. In the left panel, we split the bubble template into bubble interior and bubble shell templates. In the right panel, we split the bubble template into north bubble and south bubble templates. As in Figure 12, the correlation spectra have been normalized to a reference region; see Section 3.2.2 for details and Table 2 for a summary of the normalization factors.

(A color version of this figure is available in the online journal.)

limit (e.g., Blumenthal \& Gould 1970). Photons in the Fermi energy range can be produced by scattering of $\mathcal{O}(10-100) \mathrm{GeV}$ electrons on starlight, which is (marginally) in the $\mathrm{KN}$ regime, or by Thomson scattering of much higher-energy electrons on IR or CMB photons. Consequently, the spectral index of gamma rays might be expected to vary with latitude even if the electron spectral index is uniform, becoming harder at higher latitudes where scatterings in the Thomson limit dominate. Closer to the disk, where much of the ISRF energy density is in starlight, IC scatterings are in neither the extreme $\mathrm{KN}$ nor Thomson limits, and the spectrum needs to be computed carefully.
We consider a steady-state electron spectrum described by a power law, $d N / d E \propto E^{-\gamma}$, with energy cutoffs at $0.1 \mathrm{GeV}$ and $1000 \mathrm{GeV}$. The choice of high-energy cutoff is motivated by the local measurement of the CR electron spectrum by Fermi (Abdo et al. 2009). We consider a region $\sim 4 \mathrm{kpc}$ above the GC, as an example (and since both the WMAP haze and Fermi bubbles are reasonably well measured there), and employ the model for the ISRF used in GALPROP version 50p (Porter \& Strong 2005) at $4 \mathrm{kpc}$ above the GC. We normalize the synchrotron to the approximate value measured by $W M A P$ in the $23 \mathrm{GHz}$ $K$ band (Hooper et al. 2007), $\sim 25^{\circ}$ below the Galactic plane, 

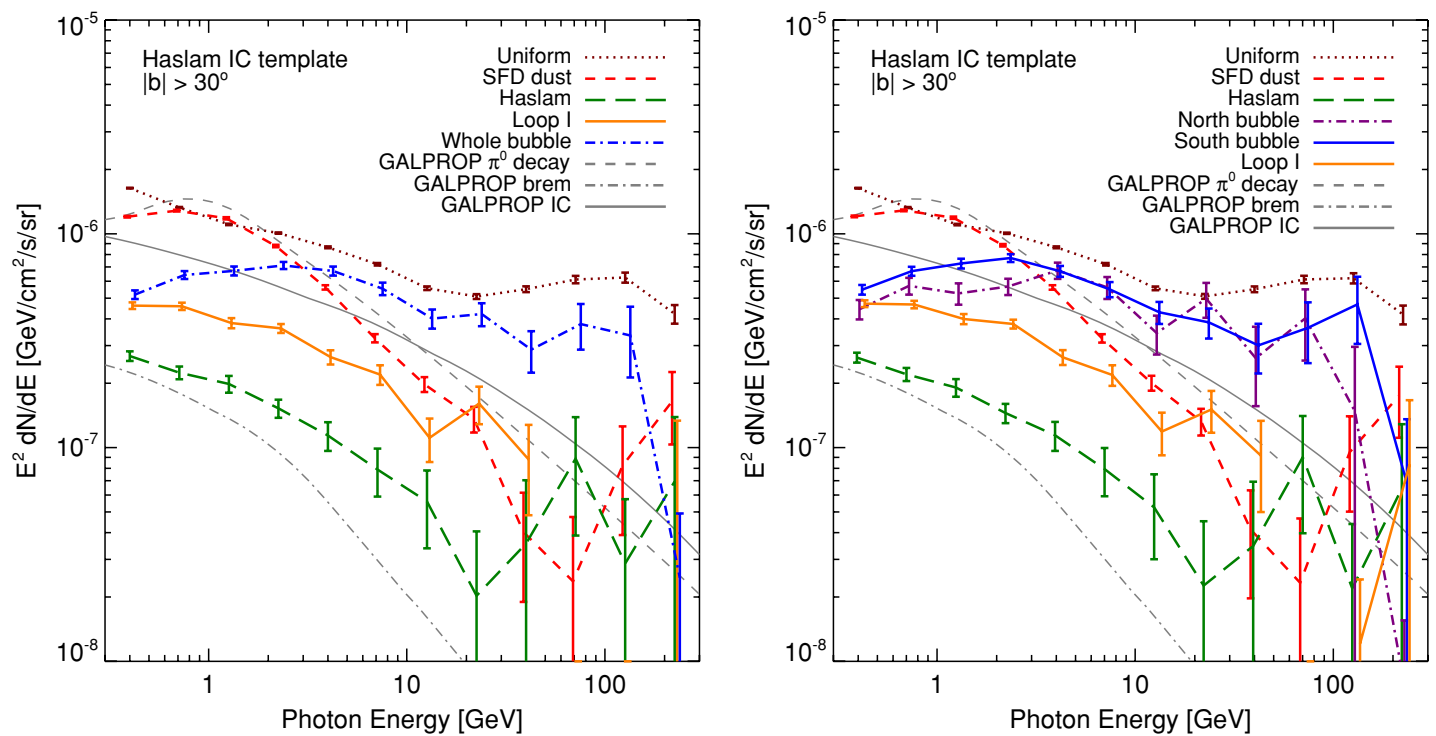

Figure 17. Same as Figure 12, but using the Haslam $408 \mathrm{MHz}$ map instead of the simple disk model as the IC template. The Haslam map contains a bright feature associated with Loop I (see Figure 18) and is dominated by synchrotron emission from softer electron CRs, of energies around $1 \mathrm{GeV}$; it is not an ideal tracer of IC emission which depends on both electron and ISRF distribution. The resulting best-fit spectrum for the bubble template remains harder than the other components, but with enhanced lower energy $(\leqslant 2 \mathrm{GeV})$ correlation coefficients compared to Figures 12 and 14 . The right panel is the same as the left panel, but with the bubble template divided into north and south bubbles (see Section 3.2.2 for more discussion). As in Figure 12, the correlation spectra have been normalized to a reference region; see Section 3.2.2 for details and Table 2 for a summary of the normalization factors.

(A color version of this figure is available in the online journal.)

and compute the corresponding synchrotron and IC spectra. The WMAP haze was estimated to have a spectrum $I_{\nu} \propto v^{-\beta}$, $\beta=0.39-0.67$ (Dobler \& Finkbeiner 2008), corresponding approximately to an electron spectral index of $\gamma \approx 1.8-2.4$; Figure 23 shows our results for a magnetic field of $10 \mu \mathrm{G}$ and $5 \mu \mathrm{G}$ at $4 \mathrm{kpc}$ above the $\mathrm{GC}$, and electron spectral indices $\gamma=1.8-3$. We find good agreement in the case of $\alpha \approx 2-2.5$, consistent with the spectrum of the WMAP haze.

In the default GALPROP exponential model for the Galactic magnetic field, $|B|=\left|B_{0}\right| e^{-z / z_{s}}$ with scale height $z_{s} \approx 2 \mathrm{kpc}$, this field strength would correspond to $B_{0} \approx 30-40 \mu \mathrm{G}$ or even higher. This value is considerably larger than commonly used (e.g., Page et al. 2007). However, models with a non-exponential halo magnetic field, as discussed by, e.g., Alvarez-Muñiz et al. (2002) and Sun et al. (2008), can have $\sim 10 \mu$ G fields well off the plane.

We note also that the extrapolated value of $B_{0}$ required to obtain good agreement between the IC and synchrotron amplitudes, in the exponential model, is somewhat higher than found by Dobler et al. (2010), who performed the comparison at $2 \mathrm{kpc}$. This apparent discrepancy originates from the fact that in the haze latitudinal profile given by Hooper et al. (2007), the emission falls off rapidly with latitude for $0>b>-15^{\circ}$, but then plateaus at $b \sim-15^{\circ}$ to $35^{\circ}$, contrary to expectations based on a $B$-field profile exponentially falling away from $z=0$. This suggests either that the magnetic field inside the bubble does not fall exponentially with $|z|$ inside the bubbles, or that the WMAP haze contains a significant free-free component at high latitude.

\subsection{Evidence of a $\sim 700 \mathrm{GeV}$ Electron Excess?}

In Figure 24, we calculate the gamma-ray spectrum from IC scattering, using the standard ISRF model taken from GALPROP - as in Figure 23, but with a different energy range for the electron CRs. An electron CR population with a hard low-energy cutoff at about $500 \mathrm{GeV}$ can fit the Fermi bubble spectrum better than a single power law extending from $0.1-1000 \mathrm{GeV}$, due to the downturn in the spectrum in the low- est energy bin. Even a rather hard $\left(d N / d E \sim E^{-2}\right)$ power-law component at $300-500 \mathrm{GeV}$ produces a long tail at low energies. Interestingly, this preferred $500-700 \mathrm{GeV}$ energy range is rather close to the peak in local $e^{+}+e^{-}$CRs observed by ATIC (Chang et al. 2008). ${ }^{14}$ We note that although the estimated error bars of energy lower than $1 \mathrm{GeV}$ mildly depend on the templates we use in the fitting procedure, the fall-off of the bubble intensity in the lowest energy bins is robust. Figure 25 shows the same analysis for 500-900 GeV electrons with two different templates for the disk IC emission, for the bubble interior and shell separately, and for the north and south bubbles. In all cases the same cut-off in the spectrum below $1 \mathrm{GeV}$ is observed.

In this case, while the lowest energy bin in the gamma rays is better fitted, the lack of low-energy CRs means that synchrotron can contribute to the WMAP haze only at the subpercent level, unless the magnetic field in the inner galaxy is extreme (and even then the spectrum does not reproduce the observations). In this case, an alternate explanation for the $W M A P$ haze would need to be considered. As suggested initially in Finkbeiner (2004a), the WMAP haze could originate from free-free emission (thermal bremsstrahlung), and this would explain the lack of a clear haze signal in WMAP polarization maps. However, the spectrum of the haze is somewhat softer than generally expected from free-free emission; the gas temperature required is also thermally unstable, requiring a significant energy injection $\left(\sim 10^{54}-10^{55} \mathrm{erg}\right)$ to maintain its temperature (Hooper et al. 2007; McQuinn \& Zaldarriaga 2010).

\subsection{Gamma-ray Power and $e^{-}$Cosmic Ray Density}

In order to estimate the total gamma-ray power emitted by the bubbles, we must estimate the surface brightness integrated over energy, the solid angle subtended, and the distance (suitably averaged). From Figure 12 we take the intensity to be

\footnotetext{
14 However, note that the large peak observed by $A T I C-2$ and $A T I C-4$ appears to be in conflict with the Fermi measurement of the spectrum, which contains no such feature.
} 

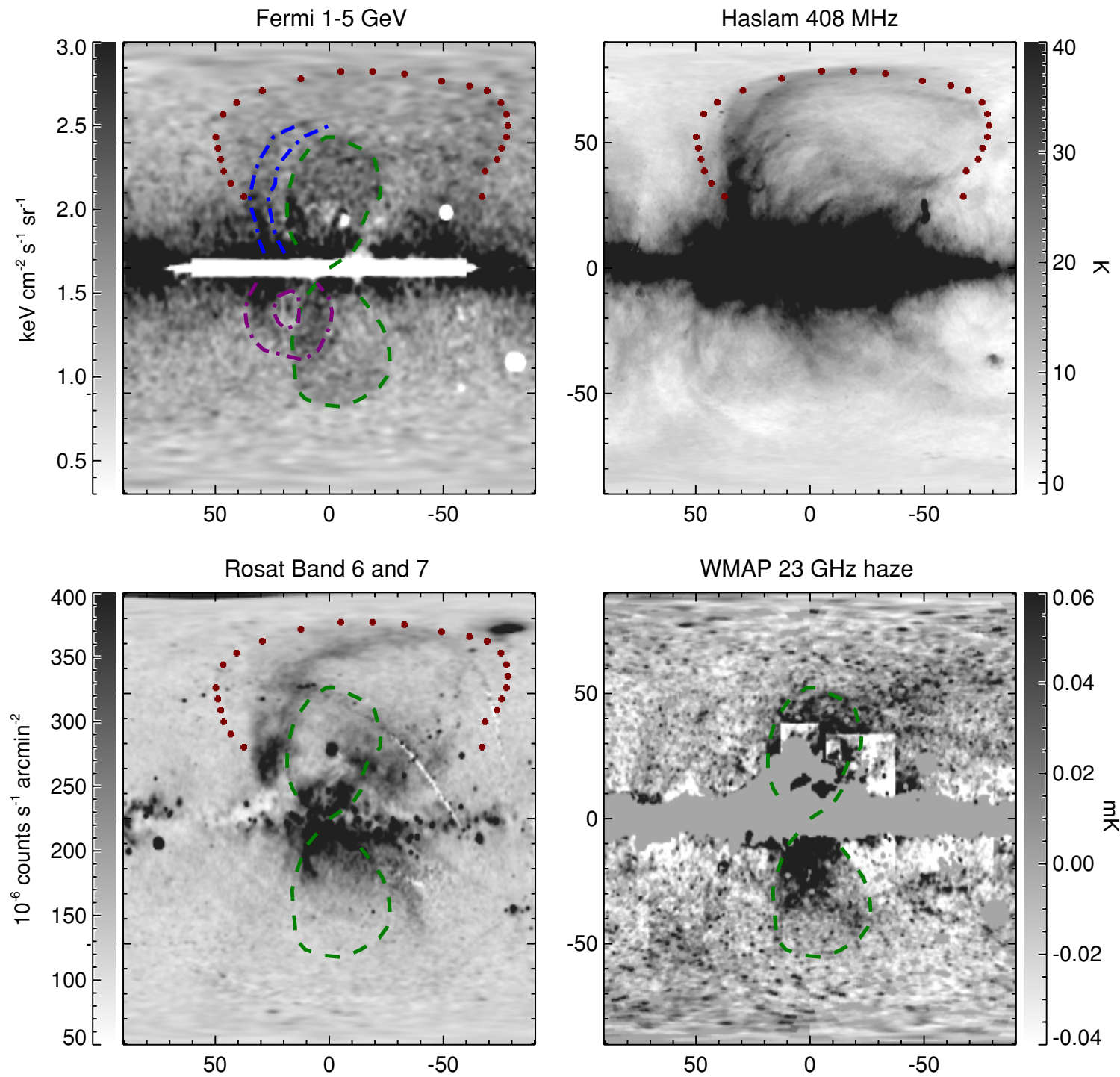

Figure 18. Comparison of the Fermi bubbles with features in other maps. Top left: point-source subtracted $1-5 \mathrm{GeV}$ Fermi-LAT 1.6 yr map, same as the lower left panel of Figure 3 with north and south bubble edges marked with green dashed line, and north arc in blue dashed line. The approximate edge of the Loop I feature is plotted in red dotted line, and the "donut" in purple dot-dashed line. Top right: the Haslam $408 \mathrm{MHz}$ map overplotted with the same red dotted line as the top left panel. The red dotted line remarkably traces the edge of the bright Loop I feature in the Haslam soft synchrotron map. Bottom left: the ROSAT $1.5 \mathrm{keV}$ X-ray map is shown together with the same color lines marking the prominent Fermi bubble features. Bottom right: WMAP haze at $K$-band $23 \mathrm{GHz}$ overplotted with Fermi bubble edges. The ROSAT X-ray features and the WMAP haze trace the Fermi bubbles well, suggesting a common origin for these features.

(A color version of this figure is available in the online journal.)

$E^{2} d N / d E=3 \times 10^{-7} \mathrm{GeV} \mathrm{cm}^{-2} \mathrm{~s}^{-1} \mathrm{sr}^{-1}$ from $1-100 \mathrm{GeV}$, integrating to $1.4 \times 10^{-6} \mathrm{GeV} \mathrm{cm}^{-2} \mathrm{~s}^{-1} \mathrm{sr}^{-1}$. The bubble template used in our analysis (Figure 3 ) subtends $0.808 \mathrm{sr}$, yielding a total bubble flux of $1.13 \times 10^{-6} \mathrm{GeV} \mathrm{cm}^{-2} \mathrm{~s}^{-1}$. To obtain an average distance for the emission, we approximate the bubbles as two spheres centered at $b= \pm 28^{\circ}$, and directly above and below the GC. For a Sun-GC distance of $8.5 \mathrm{kpc}$, this implies a distance of $9.6 \mathrm{kpc}$, and a total power (both bubbles) in the $1-100 \mathrm{GeV}$ band of $2.5 \times 10^{40} \mathrm{GeV} \mathrm{s}^{-1}$ or $4.0 \times 10^{37} \mathrm{erg} \mathrm{s}^{-1}$, which is $\sim 5 \%$ of the total Galactic gamma-ray luminosity between 0.1 and $100 \mathrm{GeV}$ (Strong et al. 2010). The electron CR density in the bubbles required to generate the observed gamma rays, at any given energy, depends strongly on the assumed electron spectrum. However, typically the required values are comparable to the locally measured electron CR density. For example, for the model in the first panel of Figure $24\left(d N / d E \propto E^{-2}\right.$ for $500 \mathrm{GeV} \leqslant E \leqslant 700 \mathrm{GeV}$ ), the inferred bubble electron density is $\sim 10 \times$ greater than the local electron density (as measured by Fermi) at an energy of $500 \mathrm{GeV}$. For a representative model from the first panel of Figure $23\left(d N / d E \propto E^{-2.3}\right.$ for $0.1 \mathrm{GeV}$ $\leqslant E \leqslant 1000 \mathrm{GeV}$, with a $10 \mu \mathrm{G}$ magnetic field generating the WMAP Haze via synchrotron), at $500 \mathrm{GeV}$ the bubble electron density is a factor $\sim 2 \times$ greater than the local density.

\section{INTERPRETATION}

As discussed in Dobler et al. (2010), the Fermi bubbles seem most likely to originate from IC scattering, since the required electron $\mathrm{CR}$ population can also naturally generate the WMAP haze as a synchrotron signal. The ROSAT X-ray measurements suggest that the bubbles are hot and hence underdense regions, and thus argue against the gamma rays originating from bremsstrahlung or $\pi^{0}$ decay.

Even though the material in the bubbles is likely high pressure, it is also probably very hot $\left(\sim 10^{7} \mathrm{~K}\right)$ and has lower gas density than the ambient ISM. This would explain why the 

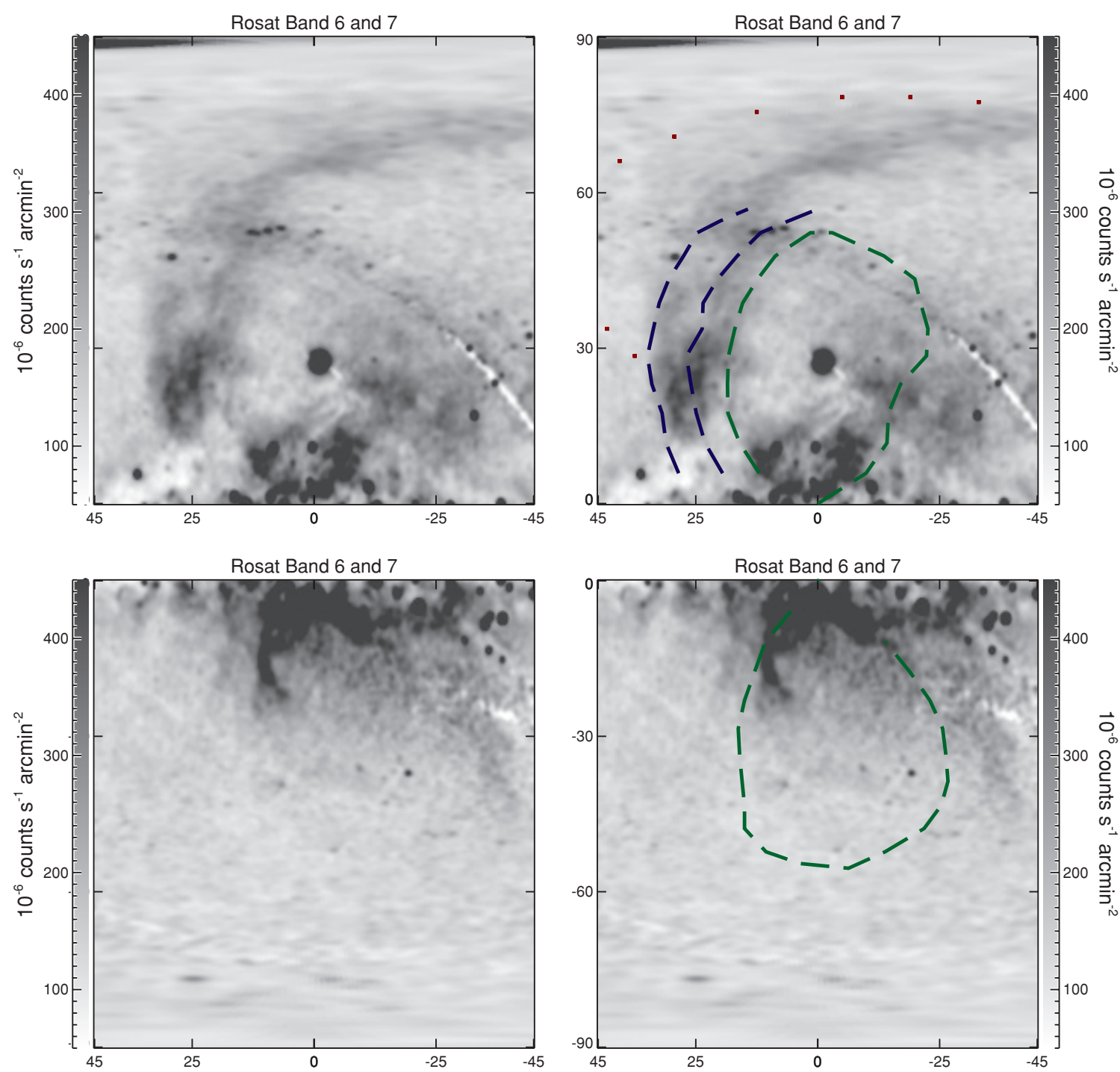

Figure 19. Top left: ROSAT X-ray haze features compared with the Fermi bubbles' morphology. Top row: the X-ray features of ROSAT band 6 and 7 in the north sky toward the GC (left panel) compared with Fermi north bubble overplotted with green dashed line, northern arc feature in blue dashed line, and Loop I feature in red dotted line (right panel). Bottom row: same as top row but for the south bubble features.

(A color version of this figure is available in the online journal.)

ROSAT $1.5 \mathrm{keV}$ map shows a "cavity" of soft X-rays toward the center of the Fermi bubble structure, like the X-ray cavity in galaxy clusters (McNamara \& Nulsen 2007), especially in the north Fermi bubble. Furthermore, allowing the Fermi bubbles to have lower density than the ambient medium means they would experience a buoyant force moving the bubble material away from the GC (see Section 6.2.3 for further discussion), which may help generate the observed morphology. Because $\pi^{0}$ and bremsstrahlung gamma-ray emission both scale as the $\mathrm{CR}$ density $\times$ the gas density, an underdense region cannot be brighter unless the CR densities are greatly increased to compensate; for protons, in particular, the propagation lengths are great enough that a proton overdensity cannot reasonably explain the sharp bubble edges observed in the data, if the bubbles are in a steady state.

If the bubbles are expanding rapidly and highly accelerated protons responsible for the gamma-ray emission are trapped behind shock fronts, then sharp edges for the Fermi bubbles could occur naturally. However, in the presence of such a shock, electrons would also be accelerated, and would generally produce more gamma rays than the protons via ICS (since the cooling time for electron CRs is much shorter than the cooling time for proton CRs of comparable energy).

It might be thought that the presence of a bright X-ray edge could lead to a sharp edge in the gamma-ray signal, via IC scattering of electron CRs on the X-ray photons. In the Thomson limit, the energy of IC scattered photons is of order $\left(\Gamma_{e} / 2\right) E_{e}$, with $\Gamma_{e}=4 E_{e} E_{\gamma} / m_{e}^{2}$ (where $E_{\gamma}$ and $E_{e}$ are the initial photon and electron energies, respectively, and $m_{e}$ is the electron mass), and the scattering cross section is independent of the initial electron and photon energies. Thus a higher-energy photon population, leading to a larger value of $\Gamma_{e}$, allows IC gamma rays at a given energy to originate from lower-energy electrons, which are much more abundant for typical electron spectra with $d N / d E \sim E^{-\gamma}, \gamma \gtrsim 2$. However, in the $\mathrm{KN}$ regime where $\Gamma_{e} \gtrsim 1$ this picture changes: the energy of scattered photons is determined mostly by the energy of the initial electron, and the cross section scales as $1 / \Gamma_{e}$. Scatterings of $\sim 50 \mathrm{GeV}$ electrons already produce $\sim 10 \mathrm{GeV}$ gamma rays; when compared to the scattering of $10 \mathrm{GeV}$ electrons on X-rays in the extreme $\mathrm{KN}$ 

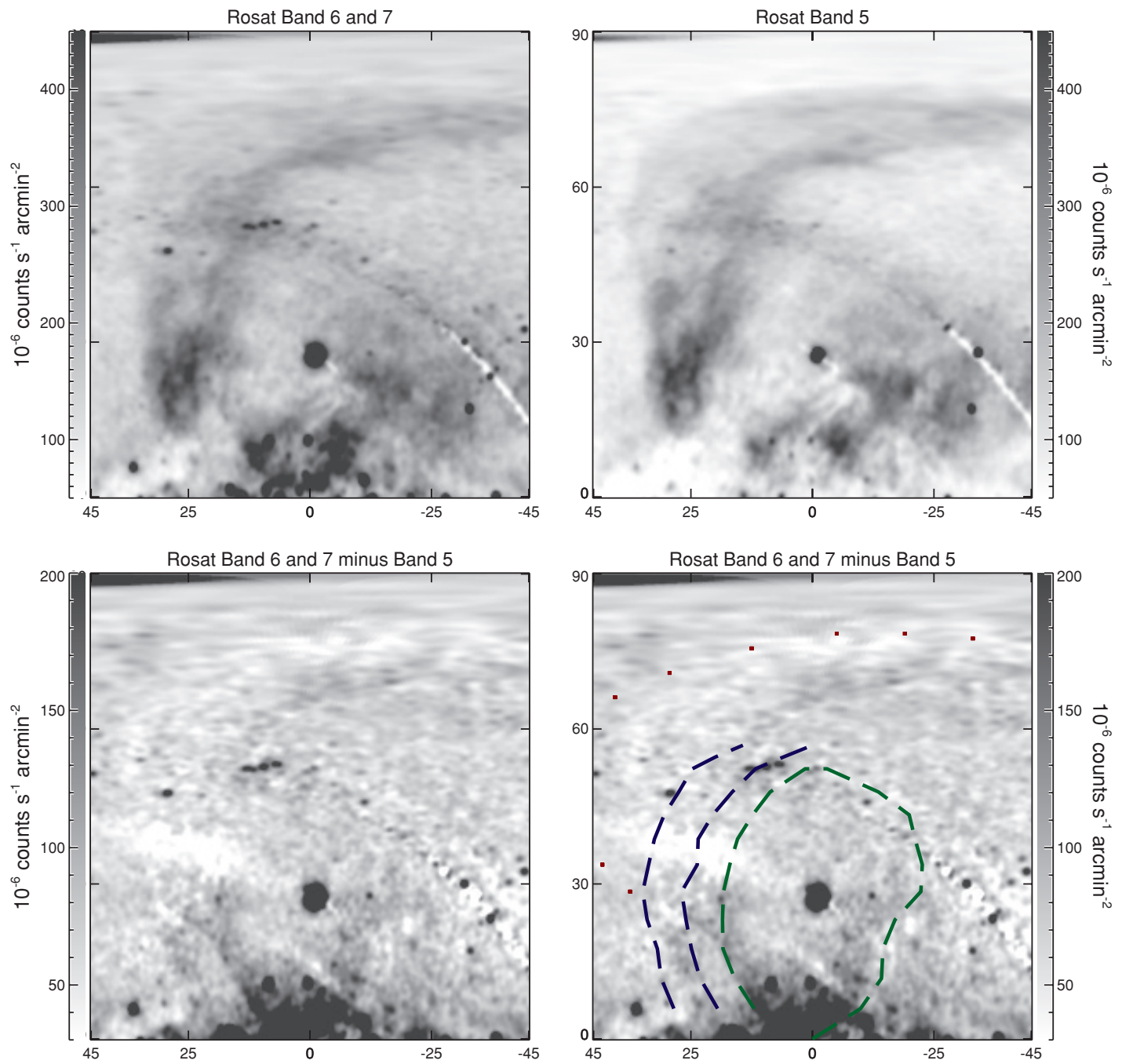

Figure 20. Top left: ROSAT X-ray features in Band 6 and 7. Top right: The same region as the left panel, but for the ROSAT X-ray map in band 5. Bottom left: The residual X-ray features after subtracting the top right softer Band 5 map from the top left harder Band 6 and 7 map. Bottom right: The same as the bottom left panel, overplotted with the Fermi bubble features, the northern arc, and Loop I features. The residual X-ray features with harder spectrum than the diffuse Loop I feature align well with the Fermi bubble structures.

(A color version of this figure is available in the online journal.)

limit, the $\mathrm{KN}$ suppression of the cross section in the latter case more than counteracts the greater abundance of $\sim 10 \mathrm{GeV}$ electrons, unless the electron spectrum is very soft (which is inconsistent with the observed signal).

Thus hard UV or X-rays in the bubbles, which might be naturally expected in a high-temperature region, would not make the IC spectrum harder, and IC scattering on these photons is subdominant to IC scattering on the usual ISRF for the electron energies in question, unless the X-ray photon number density is much greater than the starlight photon number density. Furthermore, there is no reason to think that the bubbles contain more $\lesssim 1 \mathrm{eV}$ photons, at least not in a region with a well-defined spatial edge. Thus the sharp edges of the Fermi bubbles, and the non-uniformity in the emissivity, most likely arise from the electron CR density rather than the photon density. The presence of similar sharp edges in the $W M A P$ haze (at $|b| \leqslant 30^{\circ}$ ) supports this hypothesis, if the WMAP haze is attributed to synchrotron radiation from the electron $\mathrm{CRs}$.

Similarly, the elongated shape of the Fermi bubble structures perpendicular to the Galactic plane suggests that the electron CR distribution itself is extended perpendicular to the plane. The Fermi bubble morphology is a strong argument against the possibility that the WMAP haze originates from a disk-like electron distribution with significant longitudinal variation of the magnetic field, as suggested by Kaplinghat et al. (2009).

The limb brightening of the X-rays in the ROSAT data (as shown in Figure 19), and the flat intensity profile of the Fermi bubbles, suggest the presence of a shell or shock, with increased electron CR density, coinciding with a hot thermal plasma. If the ambient medium several kpc above and below the GC were neutral, then bubbles of ionized gas could produce a void in the $\mathrm{H}$ I map (Figure 26). We see no evidence for features aligned with the bubbles in these maps, suggesting that the $\mathrm{H}$ I map in this part of the sky is dominated by disk emission, and has nothing to do with the bubbles. If the bubbles are in a static state, the bubble edges should have lower temperature than the bubble interior and thus higher gas density, although shocks or MHD turbulence might lead to higher temperatures at the bubble wall. The X-rays in ROSAT may be thermal bremsstrahlung emission, so the emissivity is proportional to the thermal electron density $x$ ion density. They could also arise from charge exchange reactions occurring when the high-speed gas in the bubbles collides with the denser gas at the bubble edge (see Snowden 2009, and reference therein); this mechanism could explain the 

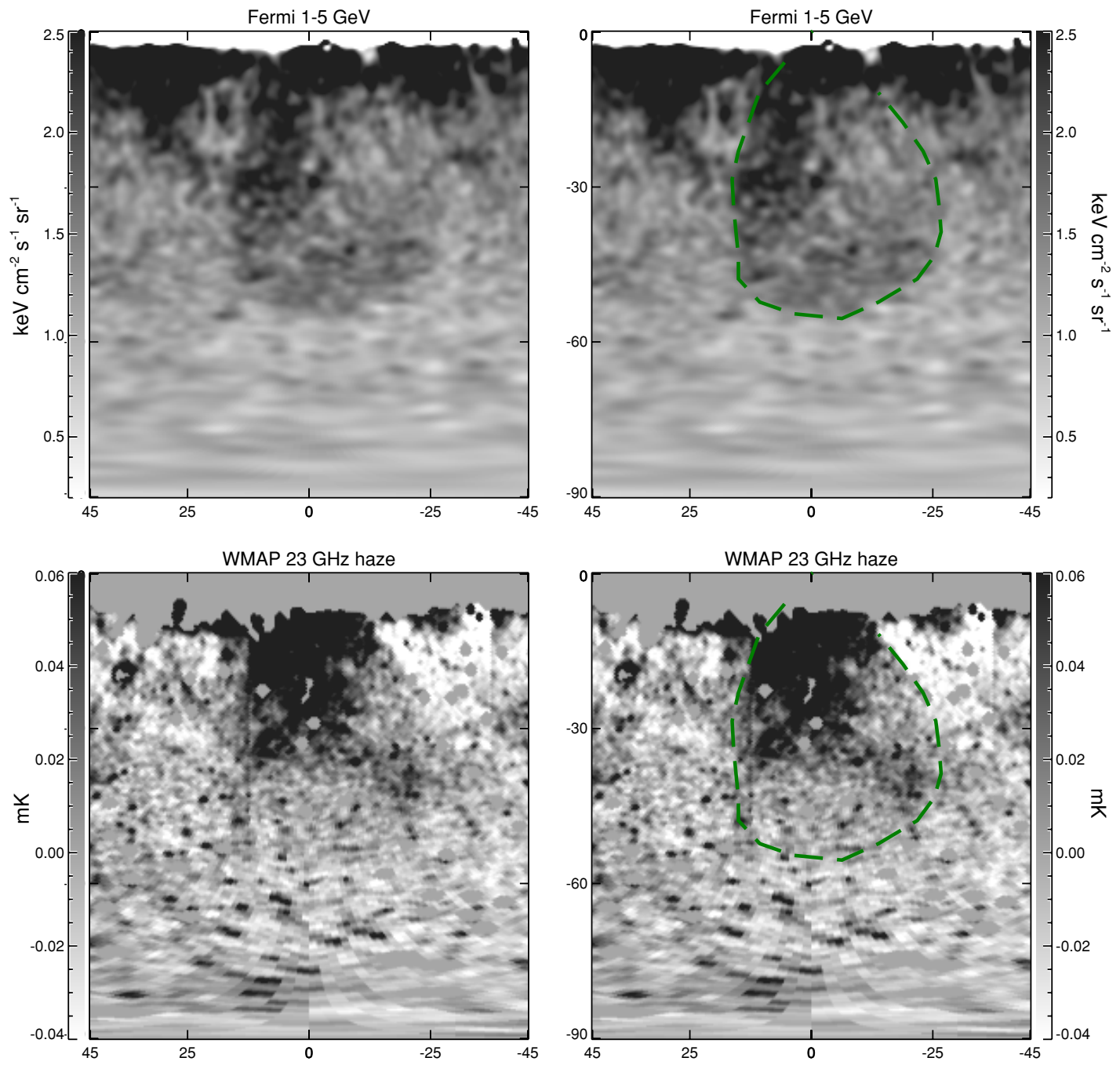

Figure 21. Fermi bubbles at $1-5 \mathrm{GeV}$ (the residual map obtained by subtracting the SFD dust map and the disk template) compared with the WMAP K-band (23 GHz) haze (Dobler \& Finkbeiner 2008). Top row: the $1-5 \mathrm{GeV}$ map with $\ell=\left[-45^{\circ}, 45^{\circ}\right]$ and $b=\left[-90^{\circ}, 0^{\circ}\right]$ (left panel) with the Fermi south bubble edge overplotted in green dashed line (right panel); the same as the left column third row panel of Figure 3. Bottom row: same sky region as top row but displaying the WMAP haze at $23 \mathrm{GHz}$ (left panel), with the Fermi south bubble edge overplotted in green dashed line (right panel).

(A color version of this figure is available in the online journal.)

pronounced limb brightening of the X-rays. As an alternative explanation, the ROSAT X-ray feature might be synchrotron emission from very high energy electron CRs. Typically, though, one needs $\sim 50 \mathrm{TeV}(\sim 5 \mathrm{TeV})$ electrons with $\sim 10 \mu \mathrm{G}(1 \mathrm{mG})$ magnetic field to produce $\sim 1 \mathrm{keV}$ synchrotron photons.

The Fermi bubble features do not appear to be associated with Loop I, a giant radio loop spanning over $100^{\circ}$ (Large et al. 1962), which is thought to be generated from the local Sco-Cen OB association. Detections of Loop I in high-energy gamma rays have been claimed by Bhat et al. (1985) and also recently by Fermi (Casandjian \& Grenier 2009); we have also discussed its presence in this work (see Section 3.2.2).

The Loop I gamma rays may be the IC counterpart of the synchrotron emission seen in the Haslam $408 \mathrm{MHz}$ map, although some of the emission might be $\pi^{0}$ gammas associated with H I (Figure 26). We compare structures identified from the Fermi 1-5 GeV maps with Loop I features in the Haslam $408 \mathrm{MHz}$ map in the top row of Figure 18, and see that the Fermi bubbles are spatially distinct from the arcs associated with Loop I; as we have shown in Figure 16, the Loop I correlated emission also has a softer spectrum than the Fermi bubble emission. The Loop I feature in the ROSAT map similarly has a softer spectrum than the limb-brightened X-ray bubble edges: as shown in Figure 20, when a low-energy map is subtracted from a higherenergy map in such a way that Loop I vanishes, the bubble edges remain bright. We also see additional shell structures which follow the Fermi bubble edges and the northern arc in the Haslam $408 \mathrm{MHz}$ map (top row of Figure 26).

The Fermi bubbles are morphologically and spectrally distinct from both the $\pi^{0}$ emission and the IC and bremsstrahlung emission from the disk electrons. As we have shown in Figures 12-17, the Fermi bubbles have a distinctly hard spectrum, $d N_{\gamma} / d E \sim E^{-2}$, with no evidence of spatial variation across the bubbles. As shown in Figure 23, an electron population with $d N_{e} / d E \sim E^{-2}-E^{2.5}$ is required to produce these gamma rays by IC scattering: this is comparable to the spectrum of electrons accelerated by SN shocks or polar cap acceleration (Biermann et al. 2010). However, diffusive propagation and cooling would be expected to soften the spectrum, making it difficult to explain the Fermi bubbles by IC scattering from a steady-state population of these electrons (a single brief injection of electrons with $d N / d E \sim E^{-2}$ could generate a sufficiently hard spectrum for the bubbles if there was a mechanism to transport them throughout the bubble without signifi- 

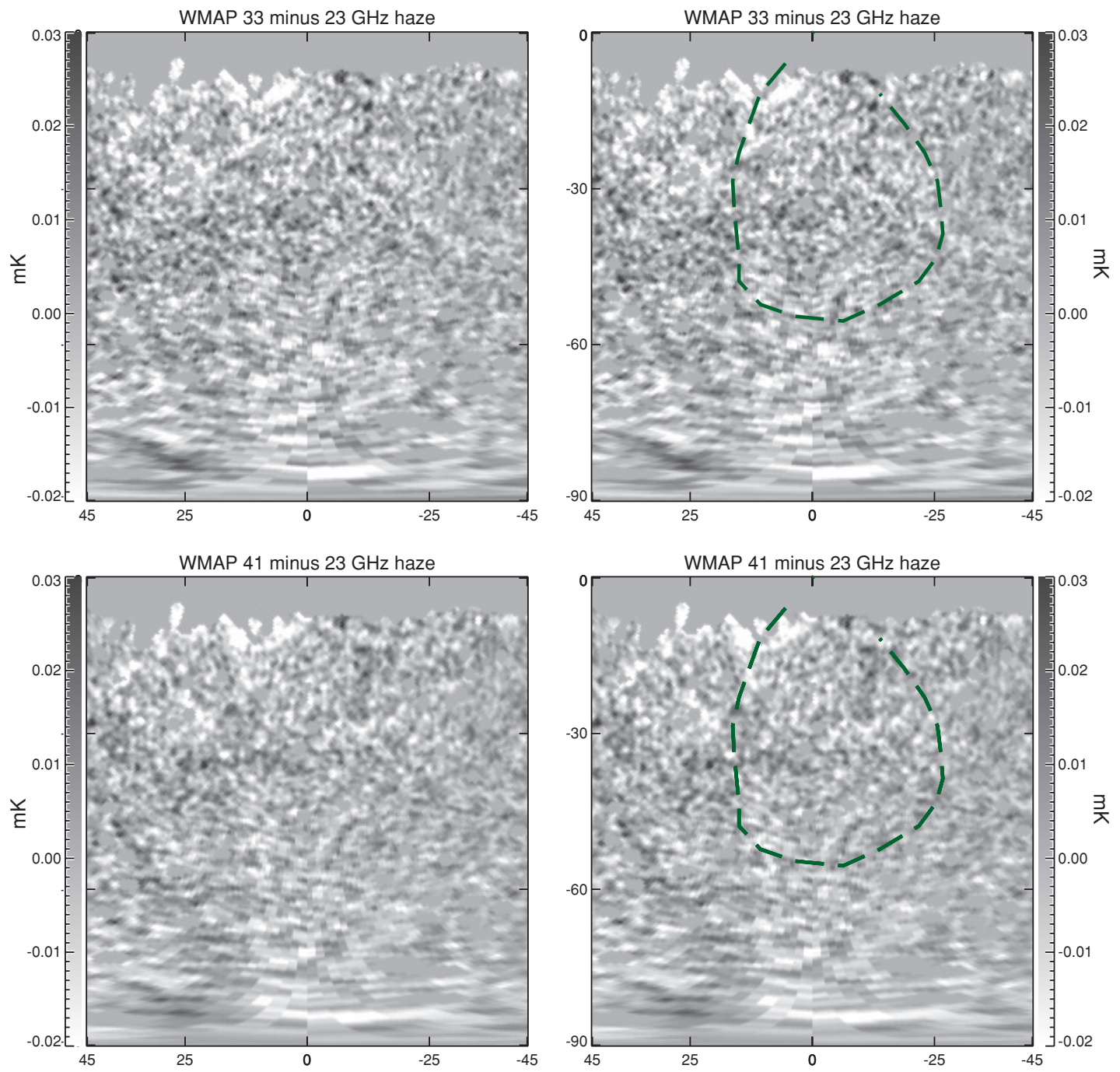

Figure 22. Difference maps of the WMAP haze. Top row: the difference map between the $23 \mathrm{GHz} W M A P$ haze and $33 \mathrm{GHz} W M A P$ haze. The right panel is the same as the left but overplotted with the south Fermi bubble edge in green dashed line. Bottom row: the same as the top row, but showing the difference between the $41 \mathrm{GHz}$ $W M A P$ haze and $33 \mathrm{GHz} W M A P$ haze. We see no apparent structure in the difference maps, indicating a consistent spectrum across different (spatial) regions of the haze.

(A color version of this figure is available in the online journal.)

cant cooling). The facts strongly suggest that a distinct electron component with a harder spectrum than steady-state supernova remnant (SNR) generated electrons is responsible for the Fermi bubbles and associated signals in the WMAP and ROSAT data.

It has been suggested that a large population of faint millisecond pulsars (MSPs) in the Milky Way halo could contribute to the WMAP and Fermi haze signals, via both pulsed gamma-ray emission and $e^{+} e^{-}$production (Malyshev et al. 2010). With a halo population of $3 \times 10^{4} \mathrm{MSPs}$, roughly half of the spin-down power going into $e^{+} e^{-}$pairs and $\sim 10 \%$ going to pulsed gamma rays, consistency with both the WMAP data and the first-year Fermi photon data is possible; however, this model does not immediately explain either the rather sharp edge in the distribution of gamma-ray emission, or the features in the ROSAT $\mathrm{X}$-ray data (the same can of course be said for models which generate the hazes via dark matter annihilation or decay). Other attempts to explain the WMAP haze with pulsars have generally employed a disk population of pulsars, either peaking in the GC or peaking at small Galactocentric radius but going to zero in the GC (Kaplinghat et al. 2009; Harding \& Abazajian 2010); such models have difficulty explaining the spherical morphol- ogy of the haze. Furthermore, as pointed out by McQuinn \& Zaldarriaga (2010), the regression of the $408 \mathrm{MHz}$ Haslam map should remove much of the contribution from young pulsars in the WMAP data, since young pulsars have a similar spatial distribution to $\mathrm{SNe}$.

How could the electron CRs possess the same hard spectrum everywhere within the bubble and extend up to $10 \mathrm{kpc}$, while experiencing a steep fall-off at the bubble edge? A large population of CRs might be entrained in large-scale Galactic outflows from the GC and enrich the bubbles (see more discussion in Section 6.3). CRs could be produced along with jets, or shock accelerated CRs from magnetic reconnection inside the bubble or near its surface (see more discussion in Section 7). However, it is challenging to produce a flat intensity profile for the bubble interior with a sharp edge. The ambient gas should be compressed to a higher density on the shell by shocks (probably also enhancing the magnetic field), and brighter synchrotron emission on the shell would then be expected, but the haze emission observed in WMAP is not limb brightened and shows no evidence for a shell of finite thickness (although we do see shell structure in the X-rays). A 

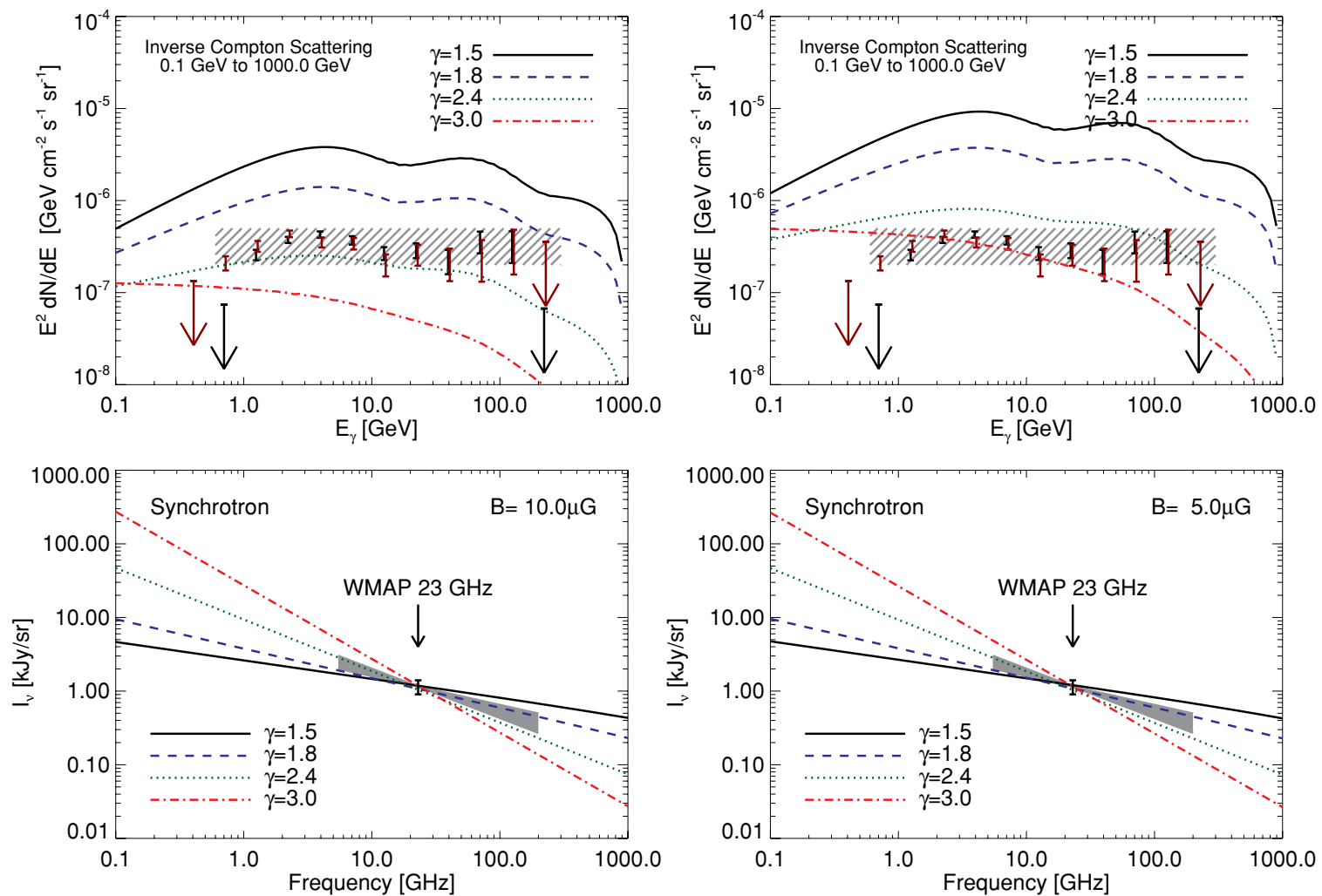

Figure 23. Estimated spectrum of IC gamma rays (upper panel) and synchrotron radiation (lower panel) originating from a hard electron spectrum along a line of sight $4 \mathrm{kpc}$ above the GC (i.e., $b \approx 25^{\circ}$ ). The steady-state electron spectrum is taken to be a power law, $d N / d E \propto E^{-\gamma}$, with index $\gamma=1.5$ (solid black), 1.8 (blue dashed), 2.4 (green dotted), and 3.0 (red dash-dotted). In all cases the spectrum has a range of $[0.1,1000] \mathrm{GeV}$. The interstellar radiation field model is taken from GALPROP version 50p, and the magnetic field is set to be $10 \mu \mathrm{G}$ for the left panel and $5 \mu \mathrm{G}$ for the right panel. The data points in the upper panels show the magnitude of the bubble emission obtained from template fitting in Figure 12 (brown) and Figure 14 (black) including the "whole bubble" template, as a function of energy. The lowest and highest bins contain $3 \sigma$ upper limits rather than data points with $1 \sigma$ error bars, due to the large uncertainties in the haze amplitude at those energies. For reference, a rectangular cross hatch region shows a approximate spectrum in the same place in this and subsequent figures. The data point in the lower panel shows the magnitude of the WMAP haze averaged over $b=-20^{\circ}$ to $-30^{\circ}$, for $|\ell|<10^{\circ}$, in the $23 \mathrm{GHz} K$ band (the overall normalization is chosen to fit this value), and the gray area indicates the range of synchrotron spectral indices allowed for the WMAP haze by Dobler \& Finkbeiner (2008). The same population of hard electrons can consistently generate the WMAP synchrotron haze and Fermi ICS bubbles.

(A color version of this figure is available in the online journal.)

schematic illustration summarizing the morphology of the Fermi bubbles and associated signals at other wavelengths is shown in Figure 27.

\section{THE ORIGIN OF THE BUBBLE STRUCTURE}

As we have shown in Section 3, the bilobular Fermi bubble structures are apparently well centered on $\ell=0^{\circ}$, and symmetric with respect to reflection across the Galactic plane (bottom row in Figure 3). The bubbles extend down to the plane, where they appear even closer to $\ell=0^{\circ}$. This alignment and north-south symmetry are unlikely unless the bubbles originate from the GC, and motivate explanations involving MBH activity (Section 6.2) or recent starbursts toward the GC (Section 6.3). We note that Sco X-1 is approximately centered on the north bubble at the present, but this appears to be a coincidence. Given the similarity between the northern and southern bubbles and the absence of any similar feature in the south, we believe that the Fermi bubbles have a GC origin.

While the origin of the Fermi bubbles is unknown, a rough estimate can be made for their age and the total energy required (although the latter quantity depends linearly on the gas density in the bubbles, which is poorly constrained). From the ROSAT data, we envisage the bubbles as hot lowdensity $\left(n \sim 10^{-2} \mathrm{~cm}^{-3}\right)$ cavities filled with $\sim 2 \mathrm{keV}$ gas, with (from the Fermi data) height $\sim 10 \mathrm{kpc}$, expanding at velocity $v \lesssim 10^{3} \mathrm{~km} \mathrm{~s}^{-1}$ : thus we estimate the energy of the Fermi bubbles to be about $10^{54}-10^{55} \mathrm{erg}$, with an age of $\sim 10^{7}\left(v / 1000 \mathrm{~km} \mathrm{~s}^{-1}\right)^{-1} \mathrm{yr}$.

Energetic galactic outflows are common phenomena which have been found in both nearby and high-redshift galaxies (Veilleux et al. 2005). Galactic winds are believed to have significant impact on galaxy formation, morphology, and their environments. The main sources of the energy are stellar winds, $\mathrm{SNe}$, and/or active galactic nuclei (AGNs); CRs and magnetic field pressure can also help drive galactic outflows (Everett et al. 2010; Socrates et al. 2008).

In this section, we present some ideas for past activity in the GC that could help to generate the shape of the Fermi gammaray bubbles and the associated signals at other wavelengths. Section 6.1 presents and discusses evidence of past GC activity. Section 6.2 discusses the hypothesis that outflows from a BH accretion event, including possible AGN jets, could form the Fermi bubbles. Section 6.3 focuses on the possibility of a previous starburst phase of the Milky Way, with the Fermi bubbles being inflated by the subsequent energetic Galactic wind.

\subsection{Observational Evidence of Previous GC Activity}

Observations across the electromagnetic spectrum have provided constraints on dynamics and evolution of the central gas 

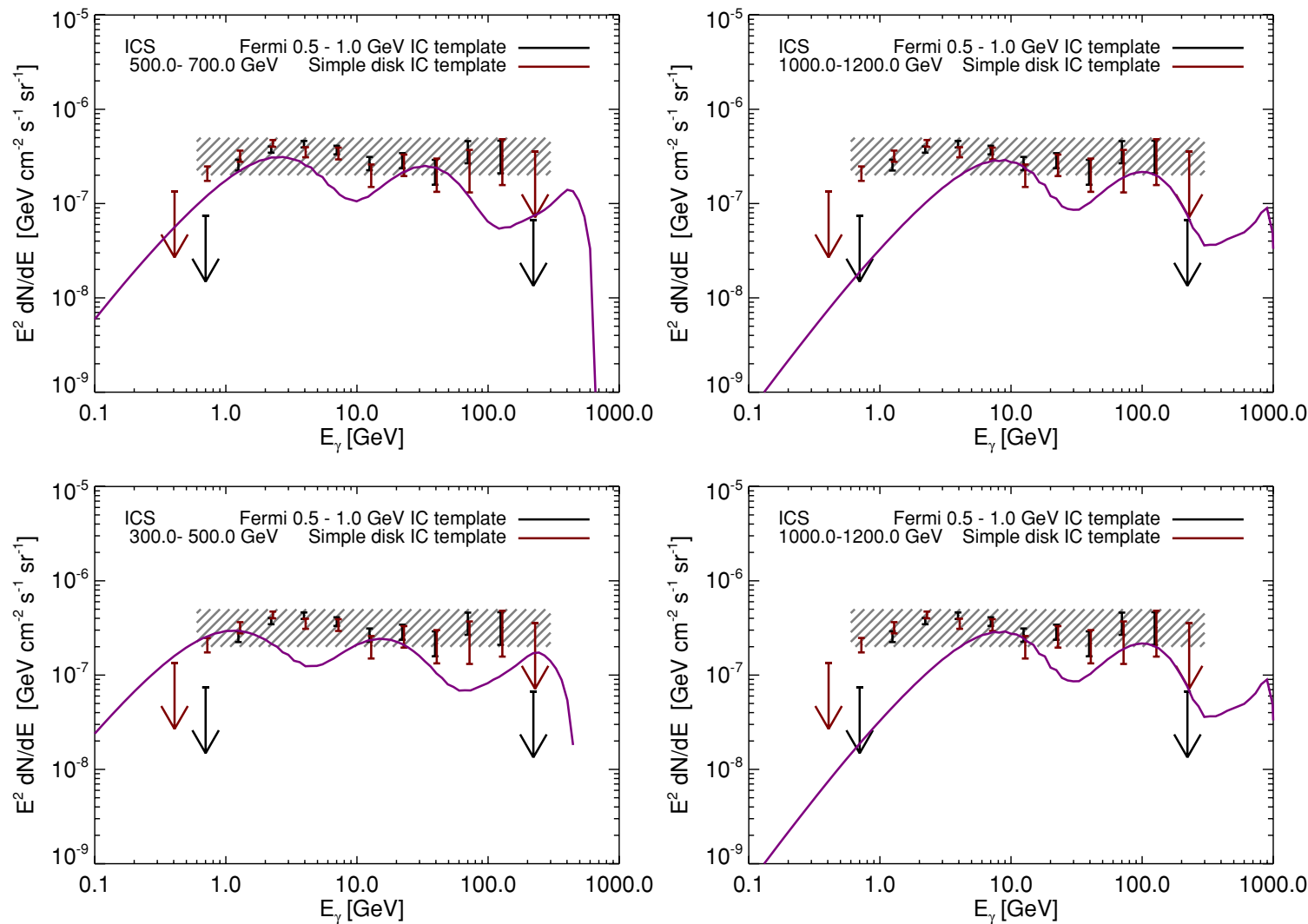

Figure 24. Estimated spectrum of IC gamma rays originating from a hard electron spectrum $\left(d N / d E \propto E^{-2}\right)$ with a limited energy range, as in the top row of Figure 23, but with different minimum and maximum energies. The normalization of the ICS signal is fitted to the data. The three peaks are from ICS of the CMB (left peak), FIR (middle peak), and optical/UV interstellar radiation field. The ISRF model is taken from GALPROP version 50p. A hard electron CR population with a low-energy cutoff at about $500 \mathrm{GeV}$ can fit the data better than a power-law electron spectrum extending from $0.1 \mathrm{GeV}$ to $1000 \mathrm{GeV}$ (see Figure 23).

(A color version of this figure is available in the online journal.)

and stellar populations, and current and prior accretion activity of the central MBH. We highlight some of the evidence of previous activities toward the GC, which may relate to the production of the Fermi bubbles.

$X$-ray reflection nebulae in the GC. There are indications of previous GC activity from X-ray echoes and time variability of reflected X-ray lines from cold iron atoms in molecular clouds around Sgr A* including Sgr B1 and B2, Sgr C, and M0.11-0.11 (Sunyaev et al. 1993; Sunyaev \& Churazov 1998). The changes in the intensity, spectrum, and morphology of the fluorescent iron nebulae near the GC, observed by ASCA and International Gamma-Ray Astrophysics Laboratory (INTEGRAL) are likely due to reflected X-rays from previous activity of Sgr A* with high luminosity $\sim 300 \mathrm{yr}$ ago. The luminosity is $\sim 1.5 \times$ $10^{39} \mathrm{erg} \mathrm{s}^{-1}$ in 2-200 keV with a power-law spectrum $d N / d E \propto$ $E^{-\gamma}$ with $\gamma=1.8 \pm 0.2$ (Ponti et al. 2010; Revnivtsev et al. 2004; Nobukawa et al. 2008). The changes in the intensities and morphologies of hard X-ray nebulosities on parsec scales have been discovered (Muno et al. 2007).

Outflows: galactic center lobe and expanding molecular ring. Sofue (2000b) and Bland-Hawthorn \& Cohen (2003b) have previously noted the presence of the extended bipolar structure in the ROSAT data, and have attributed it to a large-scale bipolar wind, powered by a starburst in the GC. In this picture, overpressured bubbles rise and expand adiabatically away from the injection region in the Galactic plane, driving shocks into the surrounding gas by ram pressure. Reflection of the shocks increases the density and temperature of the post-shocked gas, which has been suggested to have $T \approx 5 \times 10^{6} \mathrm{~K}$ and $n \approx 0.1 \mathrm{~cm}^{-3}$ at $2 \mathrm{kpc}$ above the plane (Bland-Hawthorn \& Cohen 2003b). Free-free emission in the resulting hightemperature plasma produces the observed X-ray signals. The GCL on degree scale has estimated the total kinetic energy $\sim 10^{55} \mathrm{erg}$ and a dynamical timescale of $\sim 10^{6}$ yr. The size, energy, and timescales are similar to those of the expanding molecular ring (EMR) around the GC (Kaifu et al. 1972; Scoville 1972; Totani 2006). Sofue (2000b) interpreted the North Polar Spur (NPS) with tens of degrees scale to be an outflow from the GC with an energy scale of $\sim 10^{55}-10^{56} \mathrm{erg}$ and a timescale of $\sim 10^{7}$ yr. Totani (2006) suggested that all these outflows can be attributed to the past high activity of $\mathrm{Sgr} \mathrm{A}^{*}$ of a duration of $\sim 10^{7}$ $\mathrm{yr}$, comparable to the reasonable estimation of the lifetimes of AGNs.

Diffuse X-ray emission. Muno et al. (2004) studied the diffuse $\mathrm{X}$-ray emission within $\sim 20 \mathrm{pc}$ of the GC in detail using Chandra observations. The hard component plasma with $k T \sim 8 \mathrm{keV}$ is spatially uniform and correlated with a softer component with $k T \sim 0.8 \mathrm{keV}$. Neither SNRs nor W-R/O stars are observed to produce thermal plasma hotter than $k T \sim 3 \mathrm{keV}$. A $k T \sim 8 \mathrm{keV}$ plasma would be too hot to be confined to the GC and would form a plasma wind. A large amount of energy input $\sim 10^{40} \mathrm{erg} \mathrm{s}^{-1}$ is required to sustain such hot plasma. If the hot plasma is truly diffused, the required power is too large to be explained by $\mathrm{SN}$ explosions and the origin of this hot plasma, and might be explained as a result of shock heating by the wind or AGN activities. Similar diffuse hard X-ray emission has been detected from the starburst galaxy M82 (Strickland \& Heckman 

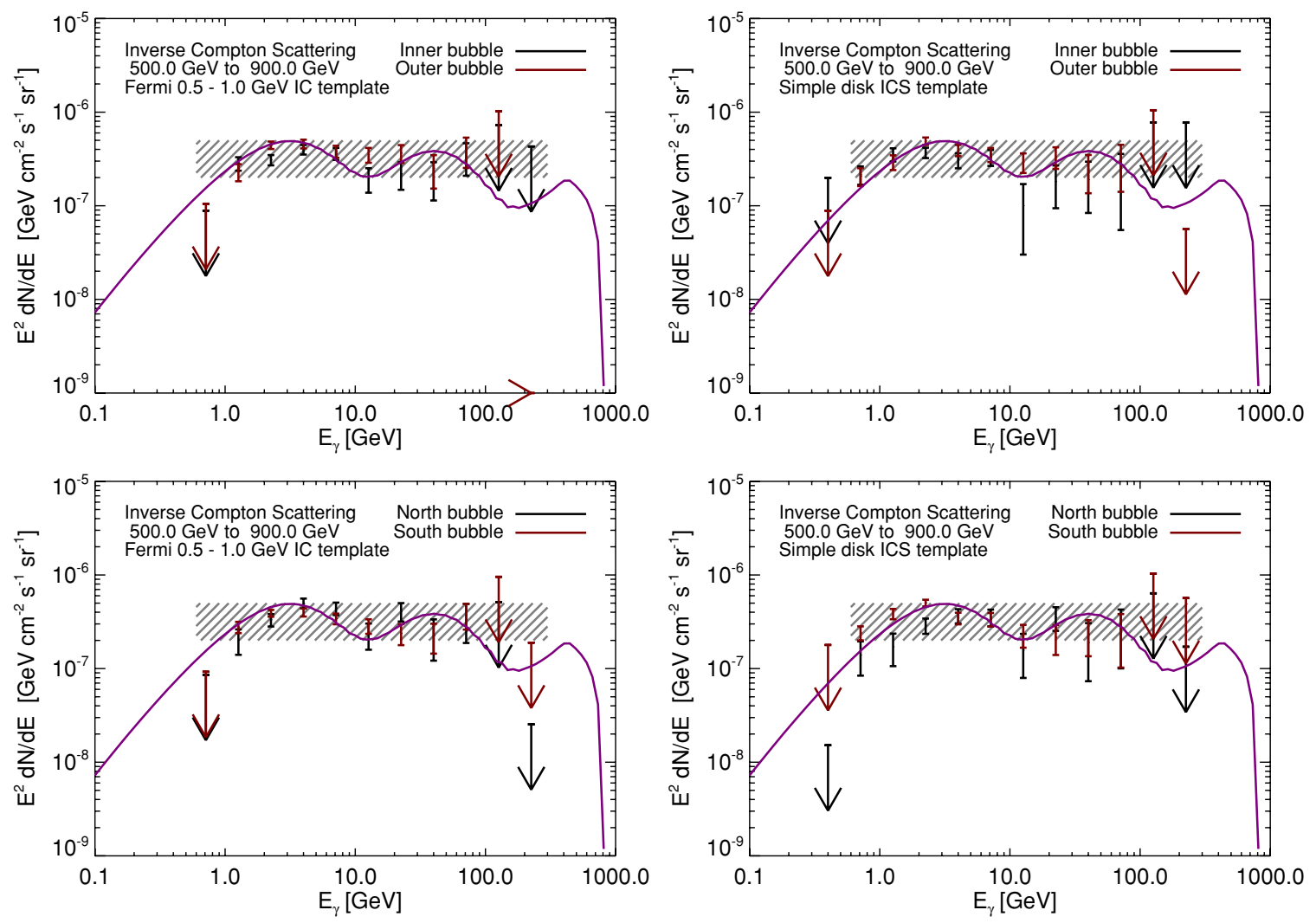

Figure 25. Estimated spectrum of IC gamma rays originating from a hard electron spectrum, the same as Figure 24, but for the 500-900 GeV energy range. Top row: data points show the separately fitted spectra for the bubble interior and outer shell templates (as defined in Figure 15), with the template for the disk IC emission given by (left panel) the $0.5-1 \mathrm{GeV}$ Fermi map with dust-correlated emission subtracted, and (right panel) the simple geometric disk model defined in Figure 3. Bottom row: as top row, but showing the separately fitted spectra for the north and south Fermi bubbles (as defined in Figure 15).

(A color version of this figure is available in the online journal.)

2007). However, Revnivtsev et al. (2009) have resolved $\sim 80 \%$ of the hard diffuse gas into faint point sources.

Bipolar hard X-ray in the GC. Chandra observations show the morphology of hot gas with a few $\mathrm{keV}$ seems to be bipolar with each lobe extending to $\sim 10 \mathrm{pc}$ (see Markoff 2010 , and reference therein). Three explanations have been suggested for the origin of the lobes: thermal wind from the central cluster of massive young stars, steady outflows from Sgr A*, or repeated episodic outbursts from Sgr A* (Markoff 2010). For the collective stellar wind interpretation, it is unclear why the lobes only extend up to $10 \mathrm{pc}$ which has a estimated flow time $\sim 3 \times 10^{4} \mathrm{yr}$, whereas the star cluster is $\sim 6$ million years old. Moreover, discrete blobs have been found within the lobes, and quasi-continuous winds are hard pressed to explain the origin of the blobs. The possibility of a transient jet-like feature is intriguing. The jets can be produced by accreting the debris of tidal disruption stars (see Section 6.2.1 for more discussion).

$O B$ stellar disk. There are two young star disks that have been identified in the central parsec of the GC (Paumard et al. 2006). In situ star formation from dense gas accretion disks is favored over the inspiraling star cluster scenario (see, e.g., Bartko et al. 2009). The gas disks could be formed as a consequence of a large interstellar cloud captured by the central $\mathrm{MBH}$, which then cooled and fragmented to form stars. Interestingly, the two star formation events happened near simultaneously about $6 \pm 2 \mathrm{Myr}$ ago and the two disks are coeval to within $\sim 1 \mathrm{Myr}$ (Paumard et al. 2006) and little activity has occurred since. The two young massive star clusters Arches and Quintuplet in the central $50 \mathrm{pc}$, with similar stellar mass, content, and mass functions, were formed $\sim 10^{7} \mathrm{yr}$ ago. It has been suggested in Paumard et al. (2006) that a global event may cause an increase in the rate of star formation, such as a passing satellite galaxy that enhanced the clouds' collision frequency and may also provide the gas for active star formation.

The unique characteristics of stellar clusters toward the GC have been used to explain the origin of the magnetized nonthermal radio filaments, threads, and streaks (LaRosa et al. 2004; Yusef-Zadeh et al. 2004). Collective winds of massive W-R and OB stars within such a dense stellar environment can produce terminal shocks that accelerate relativistic particles. The abundance and characteristics of these nonthermal radio filaments within the inner $2^{\circ}$ of the GC region can be evidence of an earlier starburst (Rosner \& Bodo 1996). Yusef-Zadeh \& Königl (2004) propose a jet model in which the characteristics common to both protostellar and extragalactic jets are used to explain the origin of nonthermal filaments in the GC region.

\subsection{Outflow from Black Hole Accretion Events}

The central MBH in our Milky Way, with an estimated mass of $M_{\mathrm{BH}} \sim 4 \times 10^{6} M_{\odot}$ (e.g., Ghez et al. 2008; Gillessen et al. 2009 ), is currently quiescent, radiating at about eight orders of magnitude lower than the Eddington luminosity ( $L_{\text {Edd }} \sim$ $10^{44} \mathrm{erg} \mathrm{s}^{-1}$ ). The X-rays of Sgr $\mathrm{A}^{*}$ are weak and thermal, in contrast to the hard nonthermal power law typically observed in most low-luminosity active galactic nuclei (LLAGNs; see, e.g., Ho 2008). Fast X-ray flaring of Sgr A* via nonthermal 

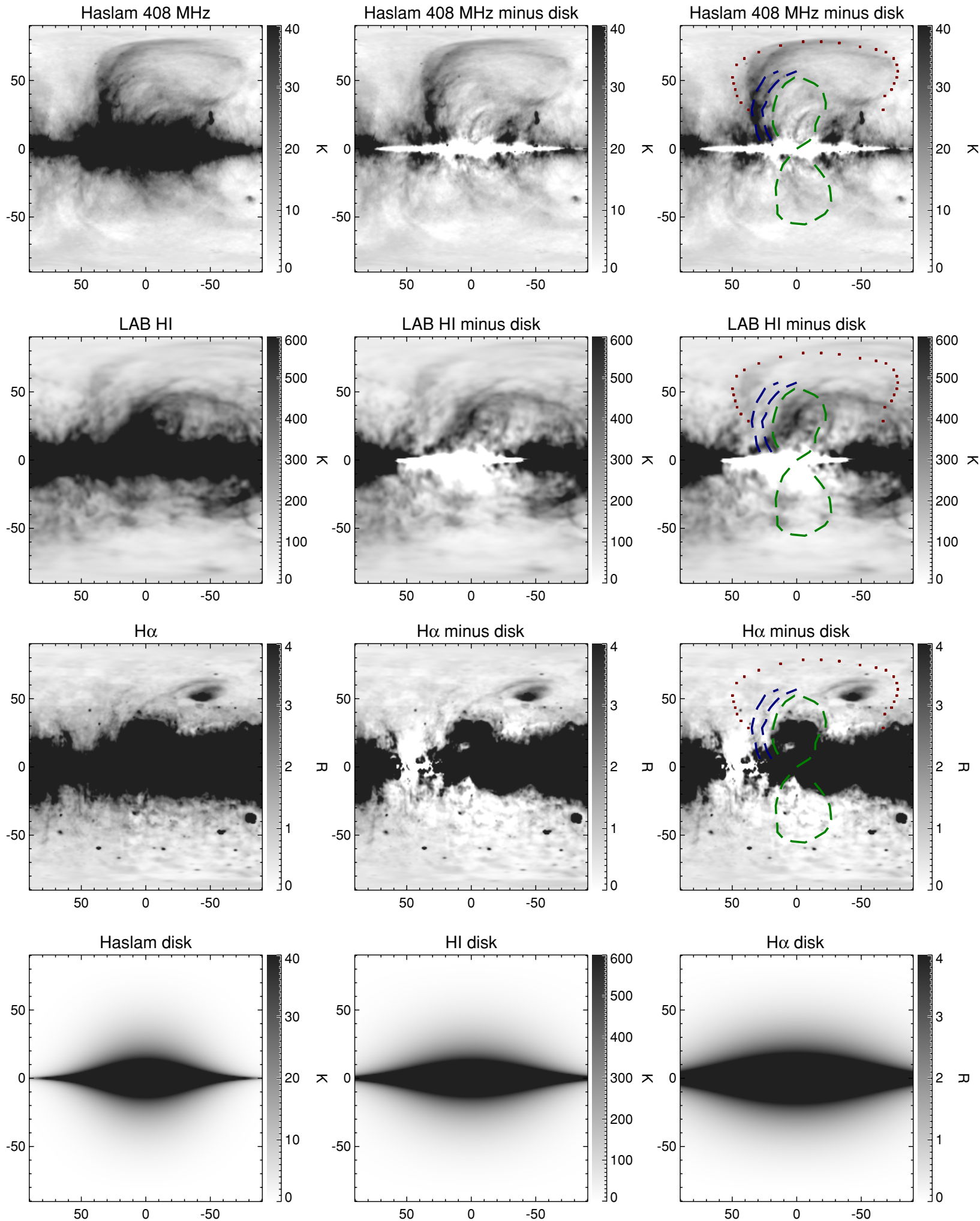

Figure 26. Fermi bubble features in other maps. Top row: the left panel shows the half-sky Haslam $408 \mathrm{MHz}$ map (Haslam et al. 1982) with $-90^{\circ}<\ell<90^{\circ}$, the middle panel subtracts a simple geometric disk template (shown in the bottom left panel) to better reveal the structures deeper into the Galactic plane. The right panel is the same as the middle panel but overplotted with the Fermi bubbles, the northern arc, and the Loop I features identified from the 1-5 GeV Fermi gamma-ray map (see Figure 4). The Loop I feature (red dotted line) align with the extended diffuse features in the Haslam $408 \mathrm{MHz}$ synchrotron map (known as North Polar Spur). The inner and outer edges of the northern arc (dashed blue lines) overlap with two arcs in the Haslam synchrotron map. However, the Fermi bubbles have no apparent counterparts in this map. Second row: the same as the top row, but for the Leiden/Argentine/Bonn (LAB) Survey of Galactic H I (Kalberla et al. 2005). The middle panel subtracts a simple disk template shown in the bottom middle panel to better reveal structures toward the GC. No apparent features have been identified that correlate with the Fermi bubbles and other features in the 1-5 GeV Fermi map (there may be some faint filaments morphologically tracing the gamma-ray features). Third row: the same as the top row but for the $\mathrm{H} \alpha$ map (Finkbeiner 2003). The middle panel subtracts a simple disk template shown in the bottom right panel to reveal more structures in the inner Galaxy. No corresponding features have been identified morphologically similar to the structures in the 1-5 GeV Fermi gamma-ray maps (color line in the right panel)

(A color version of this figure is available in the online journal.) 
processes has been discovered by Chandra ACIS observation (Baganoff et al. 2003). The observed submillimeter/IR bump is seen to flare simultaneously with the X-rays. Due to the short timescale of the flares and the lack of evidence of any standard thin accretion disk emission (Shakura \& Sunyaev 1973), a magnetic origin of the flares has been suggested, either from synchrotron or synchrotron-self Compton emission (Eckart et al. 2004; Yusef-Zadeh et al. 2006; Dodds-Eden et al. 2009). Both radiative inefficient accretion flow (RIAF) models and outflowdominated models have been invented (Falcke \& Markoff 2000; Blandford \& Begelman 1999; Yuan et al. 2002).

Clearly the MBH has not always been so underluminous: it may have experienced a long active state in the past few million or tens of million years through one or more accretion events, driving jets out of the disk, shocking the ambient material, producing both gamma rays and CRs, and appearing more similar to normal low-luminosity AGNs. If the $\mathrm{MBH}$ were radiating at the Eddington luminosity, it would take only $\sim 10^{3-4}$ years to reach the estimated energy of the Fermi bubbles; for a percent level accretion rate $\left(\sim 10^{42} \mathrm{erg} \mathrm{s}^{-1}\right)$, it would take $\sim 10^{5-6} \mathrm{yr}$, comparable to the estimated cooling time of the electron CRs.

\subsubsection{Scenarios for MBH Jet Formation in the GC}

Currently, the X-ray flares are the only unambiguous AGNlike activity that Sgr A* displays (Markoff 2010). However, the synchrotron radio emission is comparable to the typical LLAGN with compact jets in terms of spectral characteristics. It is well known that jets associated with accretion disks surrounding BHs can efficiently generate high-energy particles. BL Lacs can form relativistic jets to produce $\mathrm{TeV}$ gamma rays, as can microquasars. However, we know through multiwavelength observations that the central MBH in our Milky Way has extraordinarily low bolometric luminosity of $\sim 10^{36} \mathrm{erg} \mathrm{s}^{-1}$, and so is currently in its quiescent dim state, and no jets toward the GC have been physically resolved. Detailed examinations of the GCL have shown that the gas shell is deep into the disk, and do not support a jet origin for that structure (Law 2010). However, weak jets consistent with the spectrum of Sgr A* can be easily hidden by the blurring of their photosphere (Markoff et al. 2007).

The jets related to the energetic accretion in the GC can go in any direction; they need not be aligned with the minor axis of the Galaxy. This mechanism does not obviously provide a natural explanation for the north-south symmetry of the Fermi bubbles, and the relatively flat gamma-ray intensity inside the bubbles. Also, there is as yet no conclusive evidence for the presence of jets toward the GC (Muno et al. 2008). However, $\mathrm{CR}$ rich wide jets might have existed in the past, and the Fermi bubbles might have been inflated by the jets in a relatively short timescale without significant cooling. Although the past AGN phase could be the primary heating source of the Fermi bubbles, the low density and momentum associated with the jets do not readily distribute the thermal energy isotropically, making it harder to explain the morphology and flat intensity of the Fermi bubbles. If a starburst phase in the GC coincided with the energetic jets, $\mathrm{SNe}$ in the starburst might provide a large injection of momentum and turbulence, which could help isotropize the energy distribution.

Accretion of stars. One way to form jets in the GC is for the MBH to tidally disrupt and swallow stars in the nuclear star cluster (Hills 1975; Rees 1988). The typical picture is that when a star trajectory is sufficiently close to the $\mathrm{MBH}$, the star is captured and disrupted by tidal forces, and after about an orbital timescale, a transient accretion disk forms from the debris of the disrupted star. The capture rate of the GC MBH has been estimated at $\sim 4.8 \times 10^{-5} \mathrm{yr}^{-1}$ for main-sequence stars and $\sim 8.5 \times 10^{-6} \mathrm{yr}^{-1}$ for red giant stars. Sgr A* could temporarily behave like an AGN and produce a powerful jet by accreting the debris of such stars, which may be ejected from surrounding molecular disks. If a 50 solar mass star is captured by the $\mathrm{MBH}$ in the GC, it gives an energy in relativistic protons as high as $\sim 10^{54}-10^{55} \mathrm{erg}$ on a very short timescale $\left(\sim 10^{3}-10^{4} \mathrm{yr}\right)$, at a rate of about $\sim 10^{43} \mathrm{erg} \mathrm{s}^{-1}$.

Accretion of ISM. Quasi-periodic starbursts in the GC have been recently suggested as a result of the interactions between the stellar bar and interstellar gas (Stark et al. 2004). In this scenario, gas is driven by bar dynamics toward the inner Lindblad resonance, and accumulates in a dense gas ring at $150 \mathrm{pc}$ until the critical density threshold is reached. Then giant clouds can be formed on a short timescale, and move toward the center by dynamical friction. The timescale for this cycle to repeat is highly uncertain but is estimated to be of order $20 \mathrm{Myr}$ (Stark et al. 2004).

Accretion of IMBH. A single $10^{4}$ solar mass BH spiraling in to the GC may also trigger starbursts and change the spin of the Sgr A* Gualandris \& Merritt (2009), thereby producing precessing jets. It has been argued that one such event happens approximately every $10^{7}$ years in order to create a core of old stars in the GC, of radius $0.1 \mathrm{pc}$. Chandra has detected more than 2000 hard X-ray $(2-10 \mathrm{keV})$ point sources within $23 \mathrm{pc}$ of the GC (Muno et al. 2003). Some of them may harbor intermediatemass BHs. However, to our knowledge, no evidence of a collimated outflow has been found from the GC. There may be aligned, VLBI-scale ( $\sim 1 \mathrm{pc}$ in the GC) radio knots that have not been discovered.

Models of past enhanced Sgr A* accretion. Totani (2006) suggested an RIAF model of Sgr A* to explain both the past high X-ray luminosity and the kinetic luminosity of the outflows inferred from observations. The required boost factor of the accretion rate $\sim 10^{3}-10^{4}$ with a timescale of $\sim 10^{7} \mathrm{yr}$ is naturally expected in the model. The induced outflow is energetic enough to support the hot $(\sim 8 \mathrm{keV})$ plasma halo toward the GC. The sudden destruction of the accretion flow of $\mathrm{Sgr} \mathrm{A}^{*}$ is caused by the remnant Sgr A East passing through the $\mathrm{MBH}$ in the past $\sim 10^{2}$ yr. Such a model is claimed by Totani (2006) to better explain the positron production which is required to generate the $511 \mathrm{keV}$ line emission toward the Galactic bulge observed by INTEGRAL/SPI (Weidenspointner et al. 2008).

Galactic jets in starburst galaxies. In other galaxies, powerful AGN radio jets interacting strongly with the hot gas have been observed (McNamara \& Nulsen 2007). In relatively radio-quiet galaxies such as NGC 4636, NGC 708, and NGC 4472, (see, e.g., Wang \& Hu 2005, for a summary), faint X-ray "ghost" cavities appear without corresponding radio lobes. It has been suggested that capture of red giant stars and accretion onto the central MBH can power jets/outflows with typical energy $\sim 10^{56} \mathrm{erg}$, which is the required energy to form the observed cavities.

Interferometric monitoring of the Seyfert galaxy NGC 3079 has found evidence of radio jet components undergoing compression by collision with the clumpy ISM, within a few parsecs of the central engine. This result supports the idea that the kpcscale superbubble originates from the spread of the momentum of jets impeded from propagating freely. The generalization of 
this scenario provides an explanation for why jets in Seyfert galaxies are not able to propagate to scales of $\mathrm{kpc}$ as do jets in radio-loud AGNs (Middelberg et al. 2007).

Precessing jets. Several processes may lead to jet precession, including magnetic torques, warped disks, and gravitational torques in a binary system (see, e.g., Falceta-Gonçalves et al. 2010). If the momenta of the $\mathrm{MBH}$ and the accretion disk are not perfectly aligned, the Bardeen-Petterson effect could be a likely mechanism for precession (Bardeen \& Petterson 1975), which will force the alignment of the disk and the $\mathrm{MBH}$ angular momentum.

Irwin \& Seaquist (1988) suggested that the "bubble" structures that have been seen in nearby starburst galaxies could have been blown by a precessing VLBI-scale jet, a flat-spectrum radio core was recognized in NGC 3079 (Baan \& Irwin 1995; Irwin \& Sofue 1992). Typically, narrow, relativistic and nonprecessing jets can carve out a hot gas bubble by interaction with ISM and release most of their energy far from the GC. Wide jets with large opening angles are capable of transferring momentum into a larger area resulting in the inflation of fat bubbles (also for precessing AGN jets see Sternberg \& Soker 2008).

\subsubsection{Shocked Shells Driven by AGN Jet}

Relativistic jets dissipate their kinetic energy via interactions with the ISM. Jets in radio-loud AGNs can inflate a bubble composed of decelerated jet matter which is often referred to as a cocoon. Initially, the cocoon is highly overpressured against the ambient ISM and a strong shock is driven into the ambient matter. Then a thin shell is formed around the cocoon by the compressed medium. The shells are expected to be a promising site for particle acceleration.

As a simple estimate of the dynamics of the expanding cocoon and shell, we assume the bubbles and shells are spherical, and also assume that the ambient mass density profile has a form of a power law given by $\rho_{a}(r)=\rho_{0}(r / 1 \mathrm{kpc})^{-1.5}$, where $\rho_{0}$ is the mass density at $r=1 \mathrm{kpc}$. We further assume that the kinetic power of the jet, $L_{\mathrm{j}}$, is constant in time. Under these assumptions, the dynamics can be approximately described based on the model of stellar wind bubbles. The radius of the shock is given by $R(t) \sim 6 \rho_{0.01}^{-2 / 7} L_{42}^{2 / 7} t_{7}^{6 / 7} \mathrm{kpc}$, where $\rho_{0.01}=\rho_{0} / 0.01 m_{p} \mathrm{~cm}^{-3}$, $L_{42}=L_{\mathrm{j}} / 10^{42} \mathrm{erg} \mathrm{s}^{-1}$ and $t_{7}=t / 10^{7} \mathrm{yr}$. Taking the expected numbers for the Fermi bubbles, we get a approximate estimate of the bubble size. The total internal energy stored in the shell can be expressed as $E_{\mathrm{s}} \sim 0.1 L_{\mathrm{j}} t$, implying that roughly $10 \%$ of the total energy released by the jet is deposited in the shell.

\subsubsection{Buoyant Bubbles}

$\mathrm{X}$-ray images have revealed shock fronts and giant cavities, some with bipolar structure, in the inner regions of clusters, surrounded with X-ray emitting gas. It is believed that the power in radio jets is comparable to the energy required to suppress cooling in giant elliptical galaxies or even rich clusters (McNamara \& Nulsen 2007).

The depressions in the X-ray surface brightness of the ROSAT map may themselves indicate the presence of empty cavities or bubbles embedded in hot gas, and could be interpreted as a signature of previous AGN feedback with hot outflows. For adiabatic or supersonic bubbles first inflated by AGN jets, once the bubble reaches pressure equilibrium with the surrounding gas, it becomes buoyant and rises, because the mass density is lower in the bubble than in its surroundings. As the bubble moves away from the GC, toward regions with even lower density and pressure, it expands. The velocity at which the bubbles rise depends on the buoyancy and the drag forces.

The ISM is in turn pushed by the rising bubble, which causes a upward displacement behind the bubble called "drift." This trailing fluid can give rise to filaments of cool gas. There are indeed filamentary structures in the inner Galaxy in both $\mathrm{H}$ I and $\mathrm{H} \alpha$ maps. Their identification could support the buoyant bubble scenario.

\subsection{Nuclear Starburst}

Another possible source of dramatic energy injection is a powerful starburst in the nucleus. Starburst-induced Galactic winds are driven by the energy released by $\mathrm{SN}$ explosions and stellar winds following an intense episode of star formation, which create an overpressured cavity of hot gas. The galactic wind fluid is expected to have an initial temperature within the starburst region in the range of $10^{7}-10^{8} \mathrm{~K}$ even if it has been lightly mass loaded with cold ambient gas (Chevalier \& Clegg 1985). The ISM can be swept up by the mechanical energy of multiple SN explosions and stellar winds. Large-scale galactic outflows have been observed in starburst galaxies both in the local universe and at high redshifts (Veilleux et al. 2005; BlandHawthorn et al. 2007). Starburst episodes near the GC have been discussed in Hartmann (e.g., 1995).

\subsubsection{Morphology of Outflows in Starburst Galaxies}

Starburst-driven galactic winds have been studied extensively in both multi-waveband observations and hydrodynamical simulations (Strickland \& Stevens 2000; Veilleux et al. 2005).

AGN or starburst galaxies show bipolar outflows (Gallimore et al. 2006; Sharp \& Bland-Hawthorn 2010). The total energy of the superwind has been estimated as $\sim 10^{55}-10^{56} \mathrm{erg}$, comparable to the estimated energy of the Fermi bubbles. The Spitzer Space Telescope has found a shell-like, bipolar structure in Centaurus $A, 500 \mathrm{pc}$ to the north and south of the nucleus, in the mid-infrared (Quillen et al. 2006). The shell has been estimated to be a few million years old and its mechanical energy of $10^{53}-10^{55}$ erg depends on the expanding velocity. A small, fewthousand solar mass nuclear burst of star formation, or an AGN origin has been proposed to explain the formation of the shell.

Recently, Westmoquette et al. (2009) showed that ionized gas in the starburst core of M82 is dynamically complex with many overlapping expanding structures located at different radii, with compressed, cool, photo-ionized gas at the roots of the superwind outflow. Extra-planar warm $\mathrm{H}_{2}$ knots and filaments extending more than $\sim 3 \mathrm{kpc}$ above and below the galactic plane have also been found (e.g., Veilleux et al. 2009)

NGC 253 is one of the most famous nearby starburst galaxies and is similar to our Milky Way in its overall star formation rate, except for a starburst region toward the center of the galaxy with spatial extent of a few hundred parsecs. A galactic wind in NGC 253 was found in $\mathrm{H} \alpha$ (McCarthy et al. 1987). Strickland $\&$ Stevens (2000) discussed the spatial structure in detail in X-ray. The wind reaches out to $\sim 9 \mathrm{kpc}$ perpendicular to the disk. Filamentary structures as part of projected conical outflow are found in $\mathrm{H} \alpha$ and near-infrared $\mathrm{H}_{2}$ emission. The relatively warm gas $\left(\sim 10^{4} \mathrm{~K}\right)$ exists close to the hot gas $\left(\sim 10^{6} \mathrm{~K}\right)$. The $\mathrm{X}$-ray filaments tend to be located in inner regions compared to $\mathrm{H} \alpha$, and are brighter where the $\mathrm{H} \alpha$ emissions are locally weak. The separation between the $\mathrm{H} \alpha$ and X-ray filaments is $\sim 70 \mathrm{pc}$. The spatial distributions of $\mathrm{H} \alpha$ and X-ray indicate that the inner Galactic wind has higher temperature than the outer part. The 
UV emission seems to form a shell around the X-ray emission (Bauer et al. 2008). Very long baseline interferometry (VLBI) and Very Large Array (VLA) observations of the nuclear region of NGC 253 at $22 \mathrm{GHz}$ shows no detection of any compact continuum source on milliarcsecond scales, indicating no lowluminosity AGNs in the central region of NGC 253. It seems that the starburst region is the most plausible explanation for the source powering the wind (Brunthaler et al. 2009).

\subsubsection{The Mechanism of Galactic Winds}

The energy and momentum transfer of the Galactic wind could be dominated by high thermal and/or ram pressure. Materials have been swept-up and entrained as part of the wind, and the wind fluid comprises merged SN ejecta and massive star stellar wind material with ambient gas from the starburst region. Two popular extrinsic feedback mechanisms have been suggested: thermally driven winds powered by corecollapse $\mathrm{SNe}$ and momentum-driven winds powered by starburst radiation (Cox 2005).

On the other hand, the idea that CRs and magnetic fields can help to drive galactic winds has been known for decades (Ipavich 1975; Breitschwerdt et al. 1991). For every corecollapse $\mathrm{SN}$, about $10 \%$ of the energy release is converted into CRs $\left(\sim 10^{50} \mathrm{erg}\right)$. These CRs interact with the magnetized ISM extensively and exchange momentum through Alfvenic disturbances: the characteristic mean free path for a CR proton in the starburst phase of the GC is $\sim 1 \mathrm{pc}$. The effective cross section for $\mathrm{CR}$ protons and nuclei interacting with the ambient gas are much higher than the Thomson cross section for electrons. The luminosity at which CR collisions with gas balance gravity is about $10^{-6}$ of the usual Eddington luminosity (Socrates et al. 2008).

Momentum wind outflowing galactic supershells can also be driven by Ly $\alpha$ radiation pressure around star-forming regions (Dijkstra \& Loeb 2009). The supershell velocity can be accelerated to $10^{2}-10^{3} \mathrm{~km} \mathrm{~s}^{-1}$, and it may even be able to escape from the host galaxy. The radii are predicted to be $r_{s h}=0.1-10 \mathrm{kpc}$, with ages $t_{s h}=1-100 \mathrm{Myr}$ and energies $E_{s h}=10^{53}-10^{55} \mathrm{erg}$.

However, the morphology of the galactic wind in nearby starburst galaxies inferred from synchrotron, $\mathrm{H} \alpha$, and $\mathrm{H}$ I maps is asymmetric about both the galactic minor axis and galactic plane, which may suggest the inhomogeneous nature of the ISM. On the other hand, as we have shown, the north and south Fermi bubbles are approximately symmetric with respect to the galactic plane and the minor axis of the disk. The symmetric structure of the Fermi bubbles might indicate that they are not generated by subsequent interactions with ambient gas throughout the wind.

Furthermore, the typical speed of galactic winds is about $200-300 \mathrm{~km} \mathrm{~s}^{-1}$. It takes about $5 \times 10^{7} \mathrm{yr}$ for CR electrons to reach $10 \mathrm{kpc}$, but we have not seen any evidence of cooling in the gamma-ray intensity and spectrum. We probably need a faster transport mechanism of CRs if they were generated from the GC. However, the higher the velocity of the wind which entrains the CRs, the greater the kinetic energy the wind contains. The estimated energy of the Fermi bubbles only includes thermal energy; if they are actually kinetic energy dominated, then the energy requirement to form the Fermi bubbles is even larger (Gebauer \& de Boer 2009; Jokipii \& Morfill 1987; Lerche \& Schlickeiser 1982).

The estimated SN rate in the NGC 253 starburst region is about $0.1 \mathrm{yr}^{-1}$ (Engelbracht et al. 1998). Assuming each SN explosion releases $10^{51} \mathrm{erg}$, and $10 \%$ of the energy is transferred to heating the Fermi bubbles, this gives a rate of energy injection comparable to $\sim 10^{42} \mathrm{erg} \mathrm{s}^{-1}$. The star formation activity in NGC 253 has been underway for about $20-30 \mathrm{Myr}$, and is considered to be in a steady state for the CR transport, presumably with a smaller timescale (Engelbracht et al. 1998).

It is possible that although the center of our Milky Way is currently in its quiet phase, it was recently (in the past $10^{7} \mathrm{yr}$ ) in a starburst phase similar to NGC 253 Heesen et al. (2009). To our knowledge, however, there is no evidence of massive SN explosions $\left(\sim 10^{4}-10^{5}\right)$ in the past $\sim 10^{7}$ yr toward the GC, and no apparent Galactic wind features have been found in $\mathrm{H} \alpha$, indicating no strong recent $\left(\sim 10^{4} \mathrm{yr}\right)$ star formation activity.

Everett et al. (2008, 2010) compared the synchrotron and soft X-ray emission from large-scale galactic wind models to ROSAT and Haslam $408 \mathrm{MHz}$ maps. They show that a CR and thermally driven wind could consistently fit the observations and constrain the launching conditions of the wind, including the launching region and magnetic field strength. The comparison of the gamma-ray prediction of the wind model with Fermi-LAT diffuse emission, especially to the Fermi bubbles might have important implications for the CR-driven wind of our Milky Way.

\subsubsection{Cosmic Rays from a Starburst}

A central starburst might also generate the increased population of electron $\mathrm{CRs}$ in the $\mathrm{GeV}-\mathrm{TeV}$ energy range required to produce the gamma-ray bubble signals. Starburst galaxies host a greatly increased rate of $\mathrm{SNe}$ in the central region. The shocks from $\mathrm{SNe}$ can merge and produce energetic galactic scale winds, and the enhanced population of SNRs is believed to accelerate CRs, resulting in orders of magnitude higher CR density than currently expected in the GC. It is likely that the GC exhibited comparable CR density in the past, with a starburst phase turned on by boosted formation of massive stars. As previously discussed, during this starburst phase the CR protons produced in the inner Galaxy scatter on the ISM with very short path lengths, producing gamma rays (via $\pi^{0}$ production and decay) and electron (and positron) CRs. Although the immediately-produced gamma rays and high-density gas and ISRF associated with the starburst phase would not be observable today, the secondary electrons might be leftover from the past active starburst phase, and could have been transported to $\sim 10 \mathrm{kpc}$ by the magnetic field entrained in the Galactic winds. $\mathrm{GeV}$ and $\mathrm{TeV}$ gamma rays have recently been detected in nearby starburst galaxies NGC 253 (Acero et al. 2009), M 82, and Large Magellanic Cloud (LMC), by Fermi-LAT (Abdo et al. 2010a), H.E.S.S. (Itoh et al. 2007) and VERITAS (Karlsson 2009), support the starburst galaxies as a rich source of high-energy gamma rays. If a transient starburst did occur in the GC of our Milky Way, and produced a large population of $\mathrm{CRs}$ responsible for the observed Fermi bubbles, and WMAP haze, what triggered and terminated the starburst phase is unclear.

If the CRs are driven by winds, the halo magnetic field can carry CRs along the field lines from the inner disk/bulge into the halo, which could help the CR electrons to reach $10 \mathrm{kpc}$ without significant diffusive softening of their spectrum. The vertical CR bulk velocity is typically hundreds of $\mathrm{km} \mathrm{s}^{-1}$, which is remarkably constant over the entire extent of the disk and for galaxies with different mass. In the standard picture, CRs cannot stream faster than the Alfvén speed with respect to the static frame of the magnetic field, due to the well-known streaming instability (Kulsrud \& Pearce 1969). Considering nearby starburst galaxies, the vertical CR bulk speed has been 
measured to be $v_{\mathrm{CR}}=300 \pm 30 \mathrm{~km} \mathrm{~s}^{-1}$ for NGC 253. For a typical magnetic field strength of $B \approx 15 \mu \mathrm{G}$ and a density of the warm gas of $n \approx 0.05 \mathrm{~cm}^{-3}$ leads to an Alfvén speed of $v_{\mathrm{A}}=B / \sqrt{4 \pi \rho} \approx 150 \mathrm{~km} \mathrm{~s}^{-1}$ (Heesen et al. 2009). The super-Alfvénic CR bulk speed requires that the CRs and the magnetic field which is frozen into the thermal gas of the wind are advectively transported together. The measured CR bulk speed is the superposition $v_{\mathrm{CR}}=v_{\text {wind }}+v_{\mathrm{A}}$.

In this picture, the spectrum of electrons injected in the starburst region might be harder than elsewhere in the Galaxy if the transient starburst phase led to a top-heavy mass function of stars. Magnetic turbulence in the GC could also be much stronger than in the Solar neighborhood: the stronger the turbulence, the faster CRs are transported, and the higher the transition energy at which synchrotron/ICS losses overtake diffusive losses. It has been shown that the high star formation rate per area in the GC leads to short transport times (Becker et al. 2009).

\subsubsection{Constraints on Recent Starburst Activity}

If the Fermi bubbles were generated by previous starburst activity in the GC, we would expect to see many more SNRs toward the GC than have been discovered. Moreover, radioactive ${ }^{26} \mathrm{Al}$ (half-life $\sim 7.2 \times 10^{5} \mathrm{yr}$ ) is believed to be mainly produced by massive stars, $\mathrm{SNe}$, and novae in the Galaxy (Prantzos \& Diehl 1996). ${ }^{26} \mathrm{Al}$ decays into ${ }^{26} \mathrm{Mg}$, emitting a gamma-ray photon of $1808.65 \mathrm{keV}$. Observations of the spectrometer (SPI) on the INTEGRAL gamma-ray observatory seem to disfavor the starburst scenario: the Galaxy-wide core-collapse SN rate has been estimated at $1.9 \pm 1.1$ per century from the flux of ${ }^{26} \mathrm{Al}$ gamma rays (Diehl et al. 2006). Within its half-life, ${ }^{26} \mathrm{Al}$ can only travel $\sim 0.1 \mathrm{kpc}$ with the typical Galactic outflow velocity of $\sim 100 \mathrm{~km} \mathrm{~s}^{-1}$. One expects strong ${ }^{26} \mathrm{Al}$ gamma-ray emission concentrated toward the GC, with a flux comparable with the total gamma-ray flux from the disk, if the outflow was produced by a starburst (Totani 2006). However, such a strong concentration at the GC is not found (Prantzos \& Diehl 1996), indicating that the accretion activity of $\mathrm{Sgr} \mathrm{A}^{*}$ is more plausible as the origin of the mass outflow. Future observations of the ratio of the Galactic ${ }^{60} \mathrm{Fe}$ to ${ }^{26} \mathrm{Al}$ may provide better constraints on the starburst scenario.

\subsection{Other Ways to Generate the Bubbles}

The molecular loops in the GC could possibly be explained in terms of a buoyant rise of magnetic loops due to the Parker instability. For a differentially rotating, magnetically turbulent disk, such magnetic loops can easily be formed and rise out of the disk. The typical scale of such a loop is $1 \mathrm{kpc}$ (Machida et al. 2009).

\section{THE ORIGIN OF THE COSMIC RAYS}

It is not necessary that the physical mechanism that creates the bubbles also injects the electron CRs responsible for the Fermi bubbles. It is possible that the bubble structures were formed earlier and the electron CRs were injected by an alternative mechanism that then lights up the bubble structure with gammaray emission. In this section, we would like to separate the CR production from the bubble formation and address the spatial origin of the CRs.

In any case, the production mechanism should generate electron CRs inside the Fermi bubbles, and also prevent them from efficiently leaving the bubbles, in order to produce the observed "sharp edge." However, electrons with $\sim 10^{2} \mathrm{GeV}$ diffuse on the order of $1 \mathrm{kpc}$ before losing half their energy (McQuinn \& Zaldarriaga 2010). Higher energy electrons lose energy more rapidly and so have shorter path lengths; if the gamma-ray emission from the bubbles is dominated by IC scattering from TeV-scale electrons injected inside the bubble, then the sharp edge of the bubbles may be natural. This would in turn imply that the gamma rays observed by Fermi are largely upscattered CMB photons (starlight and far-infrared photons are upscattered to much higher energies), which is advantageous for generating such a latitudinally extended IC signal. If instead $\sim 100 \mathrm{GeV}$ electrons scattering on starlight are primarily responsible, the electron CR density must increase markedly at high Galactic latitudes to compensate for the falling of the starlight density to higher latitude. However, generating a very hard $\mathrm{CR}$ electron spectrum extending up to $\mathcal{O}(\mathrm{TeV})$ energy may be challenging for conventional CR production and acceleration mechanisms. These difficulties may be ameliorated if the hard gamma-ray signal from the Fermi bubbles is a transient rather than a steady-state solution.

\subsection{CRs from the Galactic Center}

As discussed in Section 6, the electron CRs could be produced in the inner Galaxy by mechanisms such as $\mathrm{OB}$ associations (Higdon \& Lingenfelter 2005), accretion events, and SN explosions, then entrained in subsequent jets or outflows and rapidly carried up to large scales, avoiding diffusive softening of the spectrum. Breitschwerdt \& Schmutzler (1994) suggested that the all-sky soft X-ray emission can be explained by delayed recombination in a large-scale $\mathrm{CR}$ and thermal-gas pressure driven wind. Such a wind model has been applied to the Milky Way which could explain the observed Galactic diffuse soft X-ray emission and synchrotron. The model indicates that the Milky Way may possess a kpc-scale wind.

The cooling time (denoted $\tau$ ) of $\mathrm{TeV}$-scale electron CRs can impose stringent constraints on such models. In the Thomson regime, the cooling time scales as (electron energy) ${ }^{-1}$, leading to estimates of $\tau \sim 10^{5}$ years for $\mathrm{TeV}$-scale electrons. However, where scattering on starlight photons is important, electron energies of 100-1000 GeV are no longer in the Thomson regime, so the scattering cross section is suppressed and the cooling time can be longer than naively expected.

Figure 28 shows the variation in cooling time (defined as $1 /(d \ln E / d t))$ as a function of electron energy and height above (or below) the Galactic plane, for the standard ISRF model, both with and without the inclusion of synchrotron losses (for a simple exponential magnetic field profile). ${ }^{15}$ For example, at $z=2 \mathrm{kpc}$, the cooling time for $\mathrm{TeV}$ electrons is $\sim(3-4) \times$ $10^{5}$ years, rising to $\sim 7 \times 10^{5}$ years at $z=5 \mathrm{kpc}$. Scatterings purely on the CMB give an upper bound on the lifetime of $\sim 5 \times 10^{6}(1 \mathrm{TeV} / E)$ years, but even several kpc from the $\mathrm{GC}$, scatterings on the infrared photons dominate at TeVscale energies. The effect of the $\mathrm{KN}$ cross-section suppression at higher electron energies can be seen in the small- $z$ limit where synchrotron losses are neglected, so IC scattering of the electrons on starlight is an important contribution to the total energy loss rate.

These relatively short lifetimes, especially close to the Galactic plane, may lead to severe difficulties in propagating CR electrons from the GC out to fill the bubbles. Propagation over such

\footnotetext{
15 However, note that we have treated the target photon distribution as isotropic.
} 


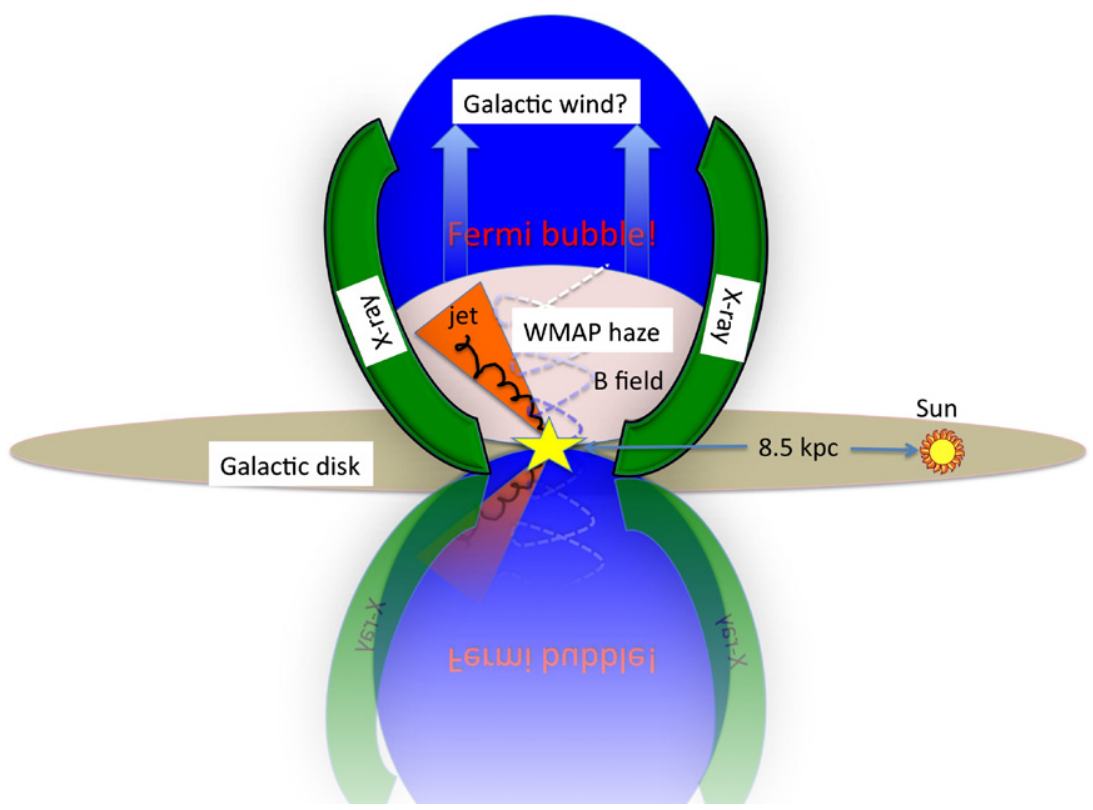

Figure 27. Schematic illustration to summarize the observations of the Fermi bubble structures. Two blue bubbles symmetric to the Galactic disk indicate the geometry of the gamma-ray bubbles observed by the Fermi-LAT. Morphologically, we see corresponding features in ROSAT soft X-ray maps, shown as green arcs embracing the bubbles. The WMAP haze shares the same edges as the Fermi bubbles (the pink egg inside the blue bubbles) with smaller extension in latitude. These related structures may have the same physical origin: past AGN activities or a nuclear starburst in the GC (the yellow star).

(A color version of this figure is available in the online journal.)

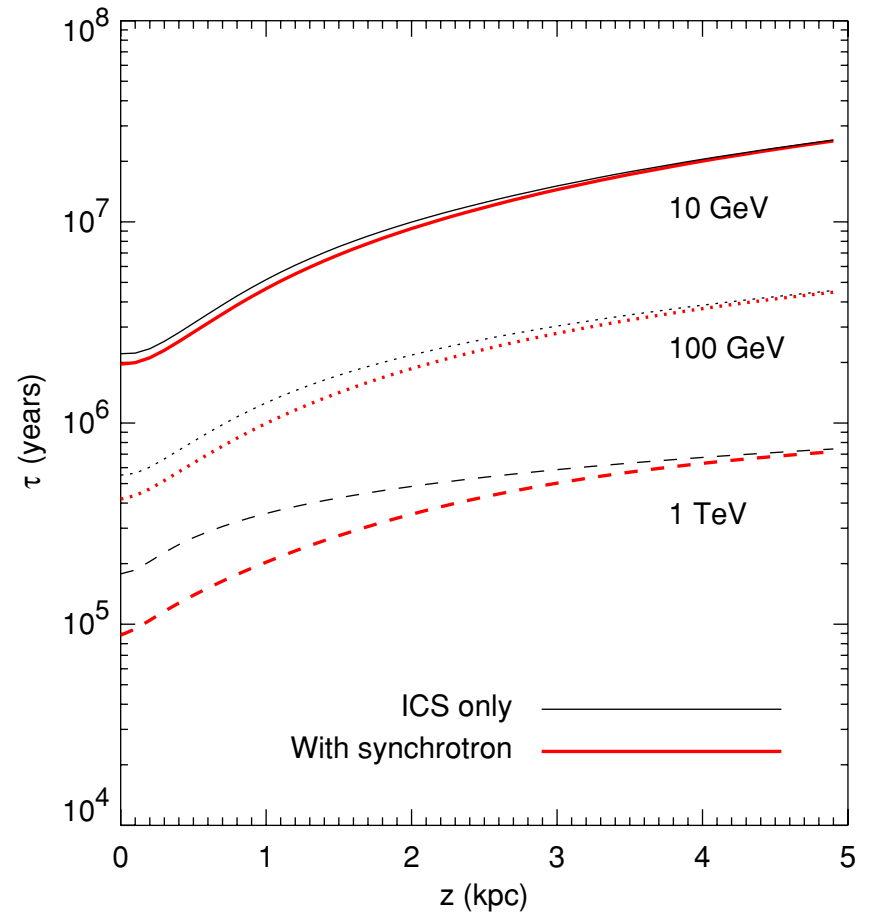

Figure 28. Cooling time for electron CRs as a function of energy and height above the Galactic plane, for $r=0$. Thin black lines: synchrotron losses are neglected; equivalently, the $B$-field is assumed to be negligible everywhere. Thick red lines: The cooling time calculation includes synchrotron losses in a magnetic field given by $|B|=30 e^{-z / 2 \mathrm{kpc}} \mu \mathrm{G}$. We use the standard radiation field model from GALPROP version $50 \mathrm{p}$, and define the cooling time $\tau=1 /(d \ln E / d t)$.

(A color version of this figure is available in the online journal.)

large distances may also lead to significant diffusive softening of the electron spectrum, which must be reconciled with the apparent spatial uniformity of the bubbles' (gamma-ray) spectral index. With electron injection primarily at the GC there is also no obvious natural explanation for the flat projected intensity profile, which seems to require sharp increases in the CR density at the bubble walls.

\subsection{CRs from the Bubble Edge}

If the majority of the electron CRs are produced from shock acceleration within the edge of the Fermi bubbles, the electron CRs in the bubble interior might be leftover CRs which undergo cooling after the shock passes through. The CRs continue to diffuse inward from the shock front while also diffusing outward; if the shock is moving faster than the electrons diffuse out, a sharp edge in the resulting Fermi bubble gamma rays is still expected. It is also possible that the CRs may be secondary electrons, produced by enhanced proton-ISM interaction in shocks (within the bubble shell), where protons could be ejected from the GC and entrained in the shocks with high gas density due to shock compression.

We can estimate the diffusion path length of $100-1000 \mathrm{GeV}$ electrons, given the lifetimes calculated in Section 7.1. We use the estimated diffusion constant from GALPROP, $K=$ $5.3 \times 10^{28} \mathrm{~cm}^{2} \mathrm{~s}^{-1}$ at a reference rigidity of $4 \mathrm{GV}$, and take the diffusion coefficient index to be $\delta=0.43$ following the results of Ahn et al. (2008). Then the path length is given by

$$
\sqrt{K \tau} \approx 1.4\left(\frac{E}{1 \mathrm{TeV}}\right)^{0.43 / 2}\left(\frac{\tau}{10^{6} \mathrm{yr}}\right)^{0.5} \mathrm{kpc} .
$$

Thus we expect the diffusion scale to be small relative to the bubble size, although not negligible.

Consequently, the electrons in the interior of the Fermi bubbles are unlikely to maintain a hard spectrum due to diffusion inward from the bubble walls. In this scenario, one needs to tune the electron CR distribution to get near-flat projected intensity. Although the Fermi bubble gamma rays along any line of sight include contributions from both the bubble interior and bubble 
shell (due to the integration along the line of sight), the flat intensity and consistent spectrum of the inner bubble and outer shell templates (Figure 16) implies that the CR spectrum in the three-dimensional bubble interior cannot be very different from the spectrum at the three-dimensional bubble shell with electron CRs generated in situ.

The edge of the Fermi bubbles might contain MHD turbulent fluid with compressed gas and magnetic field. Fast magnetic reconnection rather than shocks might drive the $\mathrm{CR}$ acceleration. Significant magnetic reversals within the bubble shell are naturally expected, just like the heliosheath region of the solar system (Drake et al. 2006; Innes et al. 1997): the crossing of the termination shock by Voyager 1 and 2 may indicate the acceleration within regions of fast magnetic reconnection (Lazarian \& Opher 2009). It is well known that magnetic field reversal can cause magnetic reconnection. When two magnetic flux tubes of different directions become too close, rearrangement of the magnetic field lines takes place, converting the energy of the magnetic field into energy of plasma motion and heating. Such phenomena have been investigated extensively in, e.g., the solar sphere and gamma-ray bursts. In the reconnection region, the energetic particles bounce between two magnetic tubes, undergoing first-order Fermi acceleration as the dominant acceleration process (Beck et al. 1996; Biskamp 1986; Elmegreen \& Scalo 2004).

On the other hand, maintaining the shape of the bubble, and preventing it from breaking out, is a non-trivial process. As the bubble rises through the ISM, it tends to fatten simply because the fluid moves faster on its sides than its front. The classical Rayleigh-Taylor instability appears at the leading edge of the bubble, as the inside density is lower than that of the ISM; Kelvin-Helmholtz instability occurs at the sides of the bubble due to discontinuities in the velocity and density. Although hydrodynamical processes may be capable of stabilizing the bubble structures against these instabilities, it has been suggested that a large-scale coherent magnetic field could help to prevent disruption. We will discuss in Section 7.3 the possibility that the magnetic field can be coherent on the Fermi bubble scale.

\subsection{CR from Diffuse Production in the Bubble}

The hard, spatially uniform spectrum of the Fermi bubbles motivates the possibility of some diffuse injection of hard CR electrons throughout the bubble volume. Such a mechanism, if present, would solve the issues of short electron cooling times, relative to the propagation time from the GC, and the hardness of the required spectrum. A uniform diffuse injection would give rise to a centrally brightened signal, so in models of this type there would most likely need to be some other mechanism increasing the electron intensity at the bubble wall, perhaps associated with a shock there.

This requirement for multiple mechanisms may seem unwieldy, and perhaps also unnecessary, if diffusion or cooling of electrons produced at the shock can explain the flat projected intensity profile and hard spectrum across the entire bubble. Given the large size of the bubble, however, it is unclear whether production or acceleration of electron CRs solely at the bubble walls can give rise to a sufficiently centrally bright and hard signal.

Decay or annihilation of dark matter has previously been proposed as a mechanism for diffuse injection of very hard electrons and positrons; in particular, dark matter annihilation has provided good fits to the data (at least in the inner $\sim 15^{\circ}-20^{\circ}$ ) in previous studies of the WMAP haze (Hooper et al. 2007;
Cholis et al. 2009b, 2009c; Harding \& Abazajian 2010; McQuinn \& Zaldarriaga 2010; Lin et al. 2010; Linden et al. 2010). The IC signal from electrons produced in dark matter annihilation would naively be expected to have approximate radial symmetry about the GC and fall off sharply at increasing $r$ and $z$, roughly tracing the distribution of dark matter density squared, but smoothed and broadened by diffusion of the electrons. This expectation is in conflict with the bubble morphology, with a gamma-ray distribution elongated perpendicular to the Galactic disk, and extending to $10 \mathrm{kpc}$ without much change in intensity. However, this naive picture is based on isotropic diffusion with a spatially uniform diffusion constant, in a tangled magnetic field; including the effects of an ordered magnetic field and anisotropic diffusion can lead to a much more bubble-like morphology (G. Dobler 2010, private communication).

Another way to produce CRs in situ inside the bubble is through magnetic reconnection. Electrons could be accelerated directly, or produced as secondaries from accelerated protons. The magnetic fields in the underdense bubbles, which may be inflated by AGN outflow, may relax into an equilibrium filling the entire volume of the bubbles. The timescale depends on the magnetization and helicity of the outflow and also the properties of the ISM. The magnetic field could undergo reconnection on a short timescale, converting magnetic energy into heat. This mechanism can explain how the bubbles could move a large distance through the ISM without breaking up. The reconnection in the bubbles can also accelerate energetic particles, circumventing the problem of synchrotron emitters having a shorter lifetime than the age of the bubble they inhabit (Braithwaite 2010). The Fermi bubbles might be initially highly turbulent, with a disordered magnetic field far from equilibrium (gas pressure and Lorentz force are not balanced); the kinetic energy would then be dissipated by viscosity, especially in the low-density bubble.

The timescale of the relaxation to equilibrium can be estimated (Braithwaite 2010) by

$$
\begin{aligned}
\tau_{\text {relax }} \approx & \frac{r}{\alpha v_{\mathrm{A}}}=\frac{r \sqrt{4 \pi \rho}}{\alpha B}=\frac{r}{\alpha}\left(\frac{4 \pi r^{3} \rho}{6 E}\right)^{\frac{1}{2}} \\
\approx & 4.4 \times 10^{6}\left(\frac{\alpha}{0.1}\right)^{-1}\left(\frac{r}{4 \mathrm{kpc}}\right)\left(\frac{\rho}{10^{-5} m_{\mathrm{p} \mathrm{cm}} \mathrm{cm}^{-3}}\right)^{\frac{1}{2}} \\
& \times\left(\frac{B}{5 \mu \mathrm{G}}\right)^{-1} \mathrm{yr},
\end{aligned}
$$

where $v_{\mathrm{A}}, B$, and $E$ are the Alfvén speed, magnetic field, and energy at equilibrium. We use the estimated characteristic values for the Fermi bubbles. The resulting timescale is on the order of $10^{6}-10^{7} \mathrm{yr}$. Furthermore, the reconnection timescale is orders of magnitude shorter than the relaxation timescale: we expect to see ongoing reconnection if equilibrium has not already been reached.

\section{POTENTIAL CONNECTIONS TO OTHER OPEN QUESTIONS}

\subsection{The Cosmic Gamma-ray Background}

Measurement of the intensity and spectrum of cosmic gammaray background (Abdo et al. 2010c; Fields et al. 2010) has suggested that instead of rare but intrinsically bright active galaxies, it is numerous but individually faint normal galaxies that comprise the bulk of the Fermi gamma-ray background. This result 
infers a tighter correlation between cosmic star formation history and the cosmic gamma-ray background (Thompson et al. 2007; Lacki et al. 2010). Galactic outflows have been identified from near-infrared observations at redshift $z \sim 2$ (Alexander et al. 2010) indicating that such outflows are common features of ultraluminous infrared galaxies. Such outflows can entrain energetic CRs to escape the galaxies, thus providing a way to contribute to the cosmic gamma-ray background at energies in the Fermi range. The Fermi bubbles may provide local evidence for such a scenario.

\subsection{The Origin of Hypervelocity Stars}

Recent surveys of hypervelocity stars (HVSs) have found 16 HVSs which are mostly B-type stars with ages $\gtrsim(1-2) \times 10^{8} \mathrm{yr}$ (see, e.g., Brown et al. 2009). These HVSs distributed in the Galactic halo are believed to originate from the GC involving interactions of stars with the MBH (Hills 1988; Yu \& Tremaine 2003). Thus energetic energy release from the GC which generate the Fermi bubbles could be dynamically related to the ejection of HVSs. Recently Lu et al. (2010) have shown that the spatial distribution of the discovered HVSs is consistent with being located on two thin disk planes. The orientation of the planes is consistent with the inner/outer clockwiserotating young stellar (CWS) disk. One possibility could be that the HVSs originate from some unknown and previously existing disk-like stellar structures. HVSs might have been ejected periodically, and related accretion events produce jets which generate the Fermi bubbles.

\subsection{The Future of the Fermi Bubbles}

What is the future of the Fermi bubbles, are they in a "breakout" stage? An interesting possibility is that the northern arc and even part of the Loop I feature are parts of the relics of previous bubbles, and the bubble production is a periodic process. The bubbles might be fast expanding shocks which might finally expand freely into the galactic halo, thus contaminating the ISM with entrained hot gas and CRs. An intriguing possibility is that CRs and gas released from previous such bubbles to the Galactic halo may contribute to the observed diffuse X-ray and gamma-ray background. In any case, the study of Fermi bubbles would have potential implications for the understanding of the feedback mechanism from the Galaxy.

\subsection{Missing Baryons and High-velocity Clouds}

$\mathrm{N}$-body/gas dynamical galaxy formation simulations have shown that for Milky Way like galaxies, about $70 \%$ extra baryonic mass should reside around the galaxy in form of hot gas (Sommer-Larsen 2006). Warm clouds are confined by the pressure of the ambient hot halo gas, which contains mass at least two orders of magnitude more than these warm clouds. The study of the Fermi bubbles may provide hints of hot gas feedback from the Galaxy, the search for the missing baryons (Bregman 2007) and the puzzle of high-velocity clouds.

\subsection{Metallicity}

Although the Galactic outflow can not inject a large fraction of the ISM, a significant amount of the freshly produced metals could be channeled along the galactic wind. In ordinary photoionization it is difficult to make the $[\mathrm{N} \mathrm{II}] / \mathrm{H} \alpha$ ratio exceed about 1.0; shock ionization/excitation is plausible once $[\mathrm{N} \mathrm{II}] / \mathrm{H} \alpha$ is detected. [N $\mathrm{II}] / \mathrm{H} \alpha$ has been estimated $\sim 1.5$ in NGC 253 , less than 1.0 in M82 where the ratio trends to increase far from the disk.

The presence of metals in the IGM has been interpreted as the consequence of energetic metal-rich outflows from galaxies. Active star formation in the inner Galaxy may contaminate the surrounding ISM, with (periodic) Galactic winds entraining the metal-rich gas to tens of kpc. The Fermi bubbles may give some hints to understanding the feedback and metallicity of the IGM. Jets from GC in general do not imply a high metallicity, and detections of metal rich outflows may essentially constrain the energetic injection from jets or Galactic outflows from previous starburst toward the GC.

\section{CONCLUSIONS}

We have identified two large gamma-ray bubbles at $1 \lesssim E \lesssim$ $50 \mathrm{GeV}$ in Fermi maps containing 1.6 years of data. They have approximately uniform surface brightness with sharp edges, neither limb brightened nor centrally brightened, and are nearly symmetric about the Galactic plane. The bubbles extend to $50^{\circ}$ above and below the GC, with a maximum width of about $40^{\circ}$ in longitude. At $|b| \lesssim 30^{\circ}$, these "Fermi bubbles" have a spatial morphology similar to the WMAP microwave haze (Finkbeiner 2004a; Dobler \& Finkbeiner 2008). The ROSAT soft X-ray $1.5 \mathrm{keV}$ map also reveals hard-spectrum features that align well with the edges of the Fermi bubbles. The similarities of the morphology and hard spectrum strongly suggest that the WMAP haze and the Fermi bubbles share a common origin.

In contrast, the Fermi bubble features are not aligned with Loop I or any other feature in the Haslam $408 \mathrm{MHz}$ map; while Loop I and other shell structures appear in the gamma rays, their spectra are softer than the bubble spectrum. Furthermore, there are no convincing features spatially correlated with the bubbles in the $\mathrm{LAB} \mathrm{H}$ I or $\mathrm{H} \alpha$ maps.

To better reveal the bubble structures, we use spatial templates to regress out known emission mechanisms. To remove $\pi^{0}$ and bremsstrahlung gammas we use the SFD dust map, assuming that the interstellar dust traces gammas produced by CR protons ( $\pi^{0}$ decay) and electrons (bremsstrahlung) colliding with the ISM. To trace the gamma rays from IC scattering of disk electrons on the ISRF, we consider three different templates: a simple geometric disk model, the $408 \mathrm{MHz}$ Haslam map, and the $0.5-1 \mathrm{GeV}$ Fermi map after removal of the (dust-correlated) $\pi^{0}$ gammas. We verify that our results are insensitive to this choice. As an additional cross check, we subtract the Fermi diffuse Galactic model from the data, finding that this also reveals the bubble structures. Although our template-fitting technique is subject to significant uncertainties due to uncertain line of sight gas and CR distributions, these uncertainties mainly affect the intensity profile at low latitudes. For $|b|>30^{\circ}$ and $1 \mathrm{GeV}<E<50 \mathrm{GeV}$ the morphology and spectrum are completely consistent for different template choices. Indeed, in the $1-5 \mathrm{GeV}$ maps, a significant part of the southern bubble is easily visible before any template subtractions.

The Fermi bubbles have an energy spectrum of $d N / d E \sim$ $E^{-2}$, significantly harder than other gamma-ray components. There is no apparent spatial variation in the spectrum between the bubble edge and interior, and the north and south bubbles have consistent spatial and spectral profiles. Both the morphology and spectrum are consistent with the two bubbles having the same origin and being the IC counterpart to the electrons which generate the microwave haze seen in WMAP (Finkbeiner 2004a; Dobler \& Finkbeiner 2008). The spectrum of the CR electrons required to generate the Fermi bubbles is harder than 
expected for electrons accelerated in SN shocks in the disk, and such disk-produced electrons would be even softer after several kpc diffusion; the morphology of the bubble structure is also quite different to that of the lower-energy electrons traced by the Haslam $408 \mathrm{MHz}$ map. Even setting aside the WMAP haze, the Fermi bubbles are unlikely to originate from excess $\pi^{0}$ emission, as (by construction) they are spatially distinct from the SFD dust map, their spectrum is much harder than that of the dust-correlated emission, and the ROSAT data suggest that the bubbles are hot and underdense rather than overdense. The morphology of the Fermi bubbles, and the overlap of the Fermi bubbles and WMAP haze, also strongly disfavor the hypothesis that a significant fraction of the high-energy gamma rays observed by Fermi in the bubble region are photons directly produced by dark matter annihilation.

The Fermi bubble structures were likely created by some large episode of energy injection in the GC, such as a past accretion event onto the central $\mathrm{MBH}$, or a nuclear starburst in the last $\sim 10 \mathrm{Myr}$. We have discussed some general possibilities and considerations in this work, and found shortcomings in each scenario; it seems likely that either significant modifications to one of these ideas, or some combination of different mechanisms, will be necessary.

Jets originating from AGN activity can potentially accelerate $\mathrm{CR}$ electrons to high energies, and transport them rapidly away from the GC; the cooling time of electrons at $100-1000 \mathrm{GeV}$ is only $10^{5-6}$ years, so if the CRs are injected and accelerated only in the GC, a very fast bulk transport mechanism is required to convey them throughout the bubbles before they lose a significant fraction of their energy. However, filling the bubbles completely, with $n=10^{-2} \mathrm{~cm}^{-3}$ gas, would require a mass injection of $\sim 10^{8}$ solar masses, so in any case it is more reasonable for the bulk of the material in the bubbles to be swept up and accelerated as the bubbles expand. Energetic shocks associated with jets can have high Mach number and thus efficiently accelerate $\mathrm{CR}$ electrons, producing hard spectra with $d N / d E \sim E^{-2}$, and the total energy required to heat the bubbles is also readily achievable by accretion events onto the central MBH.

However, the north-south symmetry of the bubbles has no obvious explanation in the context of an AGN jet: there is no reason for one jet to be oriented perpendicular to the Galactic plane. The large width and rounded shape of the bubbles are also not typical of jets, which are generally much more collimated, although a precessing jet might help explain the wide opening angle of the bubbles. If the central $\mathrm{MBH}$ becomes active on a relatively short timescale, the Fermi bubbles may be created by a number of past jets, which combine to give rise to the symmetric and uniform Fermi bubbles.

An alternate source for the large required energy injection is a nuclear starburst. The wide opening angle of the bubbles is not a problem in this case; the bubble shape is similar to that observed in NGC 3079, and the X-ray features observed by ROSAT are similar to those observed in other nearby starburst galaxies. However, no corresponding $\mathrm{H} \alpha$ signal of the Fermi bubbles is observed, in contrast to other known starburst galaxies: this problem might potentially be resolved if the $\mathrm{H} \alpha$-emitting gas has cooled in the time since the starburst phase (gas hot enough to emit the X-rays observed by ROSAT has a considerably longer cooling time). Also, generally gas filaments and clumps are observed in the X-rays in starburst galaxies, and it would seem that a relic of a past starburst should become more clumpy with gas clouds and filaments due to cooling of the gas. However, while no such structures are obvious in the ROSAT maps, the signal to noise is insufficient to place strong constraints.

The absence of any such filamentary structures inside the Fermi bubbles, on the other hand, argues against a hadronic origin for the bubble gamma-ray emission. Hadronic jets might accelerate protons to high energies, and the interactions of these protons with the ISM could then produce hard $\pi^{0}$ gammas and secondary $e^{+} e^{-}$, which would scatter on the ISRF to produce more gamma rays. In this scenario, however, the gamma-ray emission should trace the gas density, which we would not expect to be smooth and homogeneous.

Returning to the starburst scenario, the CR acceleration in this case would be due to shocks at the edge of the bipolar wind. However, the shocks expected in this scenario would be relatively weak and slow-moving, and thus may not be capable of generating a sufficiently hard electron spectrum to reproduce the signal. For example, in first-order Fermi acceleration, a shock Mach number of $\sim 3.3$ is needed to obtain an electron spectral index of 2.4, as required for the synchrotron explanation of the WMAP haze (see, e.g., Jones \& Ellison 1991).

The Fermi bubbles have sharp edges, also suggesting the presence of a shock at the bubble walls. If the CRs producing the gamma rays have a multi-kpc diffusion length (which is not expected to be the case for $1 \mathrm{TeV}$ electrons, for example), then the edges can still be sharp if the bubble edge is moving outward faster than they can diffuse. If we assume the Fermi bubbles are projected structures from three dimensional symmetric blobs toward the GC, the flat intensity profile of the bubbles requires the emissivity to rise at the bubble walls, but remain non-negligible in the bubble interior; the lack of spatial variation in the spectral index may also constrain models where the electrons diffuse long distances from an injection point. Magnetic reconnection in the interior of the bubbles, or some other mechanism such as dark matter annihilation, may help maintain a hard spectrum throughout the bubbles by accelerating existing lower-energy electrons or injecting electrons in situ.

Dark matter annihilation or decay, while an effective mechanism for injecting hard electron CRs at high latitudes, cannot produce the features in the ROSAT X-ray maps correlated with the bubbles, and would not be expected to result in sharp cutoffs in gamma-ray emission at the bubble edges. Dark matter annihilation or decay may be contributing to the bubbles, or to gamma-ray emission in the inner Galaxy that is not well subtracted by either the bubble structure template or the models for known diffuse emission mechanisms; however, understanding the Fermi bubbles will be a necessary step before extracting any such dark matter signal.

The eROSITA ${ }^{16}$ and Planck ${ }^{17}$ experiments will provide improved measurements of the X-rays and microwaves, respectively, associated with the Fermi bubbles, and so may help discriminate between these scenarios. eROSITA, on the SpectrumRoentgen-Gamma satellite which is expected to launch in 2012, will provide the first imaging all-sky survey of mid-energy $\mathrm{X}$ rays, studying the $0.2-12 \mathrm{keV}$ energy range with $\sim 100 \mathrm{eV}$ energy resolution and a PSF of $20^{\prime \prime}$. The Planck satellite, launched in 2009, will greatly improve the measurements of the WMAP haze spectrum. In addition, AMS- $02^{18}$ will launch in 2011 , and may significantly advance our understanding of CR acceleration and propagation, and help to refine our interpretation of the Fermi bubbles.

\footnotetext{
16 http://www.mpe.mpg.de/heg/www/Projects/EROSITA/main.html

17 http://www.rssd.esa.int/index.php?project=Planck

18 http://www.ams02.org
} 
We acknowledge helpful conversations with Avery Broderick, Greg Dobler, Martin Elvis, Jim Gunn, Jill Knapp, Maxim Markivitch, David Merritt, Simona Murgia, Norm Murray, Paul Nulsen, Aneta Siemiginowska, David Spergel, Anatoly Spitkovsky, and Neal Weiner. D.F. and T.S. are partially supported by NASA grant NNX10AD85G. TS is partially supported by a Sir Keith Murdoch Fellowship from the American Australian Association. This research made use of the NASA Astrophysics Data System (ADS) and the IDL Astronomy User's Library at Goddard. ${ }^{19}$

\section{REFERENCES}

Abdo, A. A., et al. 2009, Phys. Rev. Lett., 102, 181101

Abdo, A. A., et al. 2010a, ApJ, 709, L152

Abdo, A. A., et al. 2010b, Phys. Rev. Lett., 104, 101101

Abdo, A. A., et al. 2010c, ApJ, 720, 435

Acero, F., et al. 2009, Science, 326, 1080

Aharonian, F., et al. 2009, A\&A, 508, 561

Ahn, H. S., et al. 2008, Astropart. Phys., 30, 133

Alexander, D. M., Swinbank, A. M., Smail, I., McDermid, R., \& Nesvadba, N. P. H. 2010, MNRAS, 402, 2211

Alvarez-Muñiz, J., Engel, R., \& Stanev, T. 2002, ApJ, 572, 185

Atwood, W. B., et al. 2009, ApJ, 697, 1071

Baan, W. A., \& Irwin, J. A. 1995, ApJ, 446, 602

Baganoff, F. K., et al. 2003, ApJ, 591, 891

Bardeen, J. M., \& Petterson, J. A. 1975, ApJ, 195, L65

Bartko, H., et al. 2009, ApJ, 697, 1741

Bauer, M., Pietsch, W., Trinchieri, G., Breitschwerdt, D., Ehle, M., Freyberg, M. J., \& Read, A. M. 2008, A\&A, 489, 1029

Beck, R., Brandenburg, A., Moss, D., Shukurov, A., \& Sokoloff, D. 1996, ARA\&A, 34, 155

Becker, J. K., Biermann, P. L., Dreyer, J., \& Kneiske, T. M. 2009, arXiv:0901.1775

Bhat, C. L., Issa, M. R., Mayer, C. J., \& Wolfendale, A. W. 1985, Nature, 314, 515

Biermann, P. L., Becker, J. K., Caceres, G., Meli, A., Seo, E., \& Stanev, T. 2010, ApJ, 710, L53

Biskamp, D. 1986, Phys. Fluids, 29, 1520

Bland-Hawthorn, J., \& Cohen, M. 2003a, ApJ, 582, 246

Bland-Hawthorn, J., \& Cohen, M. 2003b, ApJ, 582, 246

Bland-Hawthorn, J., Veilleux, S., \& Cecil, G. 2007, Ap\&SS, 311, 87

Blandford, R. D., \& Begelman, M. C. 1999, MNRAS, 303, L1

Bloemen, H. 1989, ARA\&A, 27, 469

Blumenthal, G. R., \& Gould, R. J. 1970, Rev. Mod. Phys., 42, 237

Braithwaite, J. 2010, MNRAS, 406, 705

Bregman, J. N. 2007, ARA\&A, 45, 221

Breitschwerdt, D., McKenzie, J. F., \& Voelk, H. J. 1991, A\&A, 245, 79

Breitschwerdt, D., \& Schmutzler, T. 1994, Nature, 371, 774

Brown, W. R., Geller, M. J., Kenyon, S. J., \& Bromley, B. C. 2009, ApJ, 690, L69

Brunthaler, A., Castangia, P., Tarchi, A., Henkel, C., Reid, M. J., Falcke, H., \& Menten, K. M. 2009, A\&A, 497, 103

Casandjian, J., \& Grenier, I. (for the Fermi Large Area Telescope Collaboration) 2009, arXiv:0912.3478

Chang, J., et al. 2008, Nature, 456, 362

Chevalier, R. A., \& Clegg, A. W. 1985, Nature, 317, 44

Cholis, I., Dobler, G., Finkbeiner, D. P., Goodenough, L., Slatyer, T. R., \& Weiner, N. 2009a, arXiv:0907.3953C

Cholis, I., Dobler, G., Finkbeiner, D. P., Goodenough, L., \& Weiner, N. 2009b, Phys. Rev., D80, 123518

Cholis, I., Goodenough, L., \& Weiner, N. 2009c, Phys. Rev., D79, 123505

Cox, D. P. 2005, ARA\&A, 43, 337

Dame, T. M., Hartmann, D., \& Thaddeus, P. 2001, ApJ, 547, 792

Diehl, R., et al. 2006, Nature, 439, 45

Dijkstra, M., \& Loeb, A. 2009, MNRAS, 400, 1109

Dixon, D. D., Hartmann, D. H., Kolaczyk, E. D., Samimi, J., Diehl, R., Kanbach, G., Mayer-Hasselwander, H., \& Strong, A. W. 1998, New Astron., 3, 539

Dobler, G., \& Finkbeiner, D. P. 2008, ApJ, 680, 1222

Dobler, G., Finkbeiner, D. P., Cholis, I., Slatyer, T. R., \& Weiner, N. 2010, ApJ, 717,825

Dodds-Eden, K., et al. 2009, ApJ, 698, 676

19 Available at http://idlastro.gsfc.nasa.gov
Drake, J. F., Swisdak, M., Che, H., \& Shay, M. A. 2006, Nature, 443, 553

Eckart, A., et al. 2004, A\&A, 427, 1

Elmegreen, B. G., \& Scalo, J. 2004, ARA\&A, 42, 211

Engelbracht, C. W., Rieke, M. J., Rieke, G. H., Kelly, D. M., \& Achtermann, J. M. 1998, ApJ, 505, 639

Everett, J. E., Schiller, Q. G., \& Zweibel, E. G. 2010, ApJ, 711, 13

Everett, J. E., Zweibel, E. G., Benjamin, R. A., McCammon, D., Rocks, L., \& Gallagher, J. S., III 2008, ApJ, 674, 258

Falceta-Gonçalves, D., Caproni, A., Abraham, Z., Teixeira, D. M., \& de Gouveia Dal Pino, E. M. 2010, ApJ, 713, L74

Falcke, H., \& Markoff, S. 2000, A\&A, 362, 113

Fichtel, C. E., Hartman, R. C., Kniffen, D. A., Thompson, D. J., Ogelman, H., Ozel, M. E., Tumer, T., \& Bignami, G. F. 1975, ApJ, 198, 163

Fields, B. D., Pavlidou, V., \& Prodanovic, T. 2010, ApJ, 722, L199

Finkbeiner, D. P. 2003, ApJS, 146, 407

Finkbeiner, D. P. 2004a, ApJ, 614, 186

Finkbeiner, D. P. 2004b, arXiv:astro-ph/0409027

Gallimore, J. F., Axon, D. J., O’Dea, C. P., Baum, S. A., \& Pedlar, A. 2006, AJ, 132,546

Gebauer, I., \& de Boer, W. 2009, arXiv:0910.2027

Gehrels, N., \& Michelson, P. 1999, Astropart. Phys., 11, 277

Ghez, A. M., et al. 2008, ApJ, 689, 1044

Gillessen, S., Eisenhauer, F., Trippe, S., Alexander, T., Genzel, R., Martins, F., \& Ott, T. 2009, ApJ, 692, 1075

Gold, B., et al. 2010, arXiv: 1001.4555

Grenier, I. A., Casandjian, J., \& Terrier, R. 2005, Science, 307, 1292

Gualandris, A., \& Merritt, D. 2009, ApJ, 705, 361

Harding, J. P., \& Abazajian, K. N. 2010, Phys. Rev. D, 81, 023505

Hartmann, D. H. 1995, ApJ, 447, 646

Haslam, C. G. T., Salter, C. J., Stoffel, H., \& Wilson, W. E. 1982, A\&AS, 47, 1

Heesen, V., Beck, R., Krause, M., \& Dettmar, R. 2009, A\&A, 494, 563

Higdon, J. C., \& Lingenfelter, R. E. 2005, ApJ, 628, 738

Hills, J. G. 1975, AJ, 80, 809

Hills, J. G. 1988, Nature, 331, 687

Ho, L. C. 2008, ARA\&A, 46, 475

Hooper, D., Finkbeiner, D. P., \& Dobler, G. 2007, Phys. Rev. D, 76, 083012

Innes, D. E., Inhester, B., Axford, W. I., \& Wilhelm, K. 1997, Nature, 386, 811

Ipavich, F. M. 1975, ApJ, 196, 107

Irwin, J. A., \& Seaquist, E. R. 1988, ApJ, 335, 658

Irwin, J. A., \& Sofue, Y. 1992, ApJ, 396, L75

Itoh, C., et al. 2007, A\&A, 462, 67

Jokipii, J. R., \& Morfill, G. 1987, ApJ, 312, 170

Jones, F. C., \& Ellison, D. C. 1991, Space Sci. Rev., 58, 259

Kaifu, N., Kato, T., \& Iguchi, T. 1972, Nature, 238, 105

Kalberla, P. M. W., Burton, W. B., Hartmann, D., Arnal, E. M., Bajaja, E., Morras, R., \& Pöppel, W. G. L. 2005, A\&A, 440, 775

Kaplinghat, M., Phalen, D. J., \& Zurek, K. M. 2009, J. Cosmol. Astropart. Phys., JCAP12(2009)010

Karlsson, N. (for the VERITAS collaboration) 2009, arXiv:0912.3807

Kniffen, D. A., \& Fichtel, C. E. 1981, ApJ, 250, 389

Koyama, K., Maeda, Y., Sonobe, T., Takeshima, T., Tanaka, Y., \& Yamauchi, S. 1996, PASJ, 48, 249

Kulsrud, R., \& Pearce, W. P. 1969, ApJ, 156, 445

Lacki, B. C., Thompson, T. A., Quataert, E., Loeb, A., \& Waxman, E. 2010, arXiv: 1003.3257

Large, M. I., Quigley, M. J. S., \& Haslam, C. G. T. 1962, MNRAS, 124, 405

LaRosa, T. N., Nord, M. E., Lazio, T. J. W., \& Kassim, N. E. 2004, ApJ, 607, 302

Law, C. J. 2010, ApJ, 708, 474

Lazarian, A., \& Opher, M. 2009, ApJ, 703, 8

Lerche, I., \& Schlickeiser, R. 1982, A\&A, 107, 148

Lin, T., Finkbeiner, D. P., \& Dobler, G. 2010, Phys. Rev. D, 82, 023518

Linden, T., Profumo, S., \& Anderson, B. 2010, Phys. Rev. D, 82, 063529

Lu, Y., Zhang, F., \& Yu, Q. 2010, ApJ, 709, 1356

Machida, M., et al. 2009, PASJ, 61, 411

Malyshev, D., Cholis, I., \& Gelfand, J. D. 2010, ApJ, 722, 1939

Markoff, S. 2010, Proc. Natl. Acad. Sci., 107, 7196

Markoff, S., Bower, G. C., \& Falcke, H. 2007, MNRAS, 379, 1519

McCarthy, P. J., van Breugel, W., \& Heckman, T. 1987, AJ, 93, 264

McNamara, B. R., \& Nulsen, P. E. J. 2007, ARA\&A, 45, 117

McQuinn, M., \& Zaldarriaga, M. 2010, arXiv:1004.1189

Middelberg, E., Agudo, I., Roy, A. L., \& Krichbaum, T. P. 2007, MNRAS, 377, 731

Morris, M., \& Serabyn, E. 1996, ARA\&A, 34, 645

Muno, M. P., Baganoff, F. K., Brandt, W. N., Morris, M. R., \& Starck, J. 2008, ApJ, 673, 251 
Muno, M. P., Baganoff, F. K., Brandt, W. N., Park, S., \& Morris, M. R. 2007, ApJ, 656, L69

Muno, M. P., et al. 2003, ApJ, 589, 225

Muno, M. P., et al. 2004, ApJ, 613, 326

Nobukawa, M., et al. 2008, PASJ, 60, 191

Page, L., et al. 2007, ApJS, 170, 335

Paumard, T., et al. 2006, ApJ, 643, 1011

Ponti, G., Terrier, R., Goldwurm, A., Belanger, G., \& Trap, G. 2010, ApJ, 714, 732

Porter, T. A., \& Strong, A. W. 2005, Proc. 29th ICRC, 4, 77

Prantzos, N., \& Diehl, R. 1996, Phys. Rep., 267, 1

Quillen, A. C., Bland-Hawthorn, J., Brookes, M. H., Werner, M. W., Smith, J. D., Stern, D., Keene, J., \& Lawrence, C. R. 2006, ApJ, 641, L29

Rees, M. J. 1988, Nature, 333, 523

Revnivtsev, M., Sazonov, S., Churazov, E., Forman, W., Vikhlinin, A., \& Sunyaev, R. 2009, Nature, 458, 1142

Revnivtsev, M. G., et al. 2004, A\&A, 425, L49

Rosner, R., \& Bodo, G. 1996, ApJ, 470, L49

Schlegel, D. J., Finkbeiner, D. P., \& Davis, M. 1998, APJ, 500, 525

Scoville, N. Z. 1972, ApJ, 175, L127

Shakura, N. I., \& Sunyaev, R. A. 1973, A\&A, 24, 337

Sharp, R. G., \& Bland-Hawthorn, J. 2010, ApJ, 711, 818

Smialkowski, A., Wolfendale, A. W., \& Zhang, L. 1997, Astropart. Phys., 7, 21

Snowden, S. L. 2009, Space Sci. Rev., 143, 253

Snowden, S. L., et al. 1997, ApJ, 485, 125

Socrates, A., Davis, S. W., \& Ramirez-Ruiz, E. 2008, ApJ, 687, 202

Sofue, Y. 2000a, ApJ, 540, 224

Sofue, Y. 2000b, ApJ, 540, 224

Sofue, Y. 2003, PASJ, 55, 445

Sommer-Larsen, J. 2006, ApJ, 644, L1

Stark, A. A., Martin, C. L., Walsh, W. M., Xiao, K., Lane, A. P., \& Walker, C. K. 2004, ApJ, 614, L41

Sternberg, A., \& Soker, N. 2008, MNRAS, 389, L13
Strickland, D. K., \& Heckman, T. M. 2007, ApJ, 658, 258

Strickland, D. K., \& Stevens, I. R. 2000, MNRAS, 314, 511

Strong, A. W. 1984, Adv. Space Res., 3, 87

Strong, A. W., Mayer-Hasselwander, H. A., Bloemen, J. B. G. M., Hermsen, W., \& Lebrun, F. 1987, A\&AS, 67, 283

Strong, A. W., \& Moskalenko, I. V. 1999, Proc. 26th ICRC, 4, 255

Strong, A. W., Moskalenko, I. V., Porter, T. A., Jóhannesson, G., Orlando, E., \& Digel, S. W. 2009, arXiv:0907.0559

Strong, A. W., Moskalenko, I. V., \& Ptuskin, V. S. 2007, Annu. Rev. Nucl. Part. Sci., 57,285

Strong, A. W., et al. 2010, ApJ, 722, L58

Sun, X. H., Reich, W., Waelkens, A., \& Enßlin, T. A. 2008, A\&A, 477, 573

Sunyaev, R., \& Churazov, E. 1998, MNRAS, 297, 1279

Sunyaev, R. A., Markevitch, M., \& Pavlinsky, M. 1993, ApJ, 407, 606

Thompson, T. A., Quataert, E., \& Waxman, E. 2007, ApJ, 654, 219

Thompson, T. A., Quataert, E., Waxman, E., Murray, N., \& Martin, C. L. 2006, ApJ, 645, 186

Totani, T. 2006, PASJ, 58, 965

Veilleux, S., Cecil, G., \& Bland-Hawthorn, J. 2005, ARA\&A, 43, 769

Veilleux, S., Rupke, D. S. N., \& Swaters, R. 2009, ApJ, 700, L149

Wang, J., \& Hu, C. 2005, ApJ, 630, L125

Weidenspointner, G., et al. 2008, New Astron. Rev., 52, 454

Westmoquette, M. S., Smith, L. J., Gallagher, J. S., Trancho, G., Bastian, N., \& Konstantopoulos, I. S. 2009, ApJ, 696, 192

Yu, Q., \& Tremaine, S. 2003, ApJ, 599, 1129

Yuan, F., Markoff, S., \& Falcke, H. 2002, A\&A, 383, 854

Yusef-Zadeh, F., Hewitt, J. W., \& Cotton, W. 2004, ApJS, 155, 421

Yusef-Zadeh, F., \& Königl, A. 2004, in ASP Conf. Ser. 322, The Formation and Evolution of Massive Young Star Clusters, ed. H. J. G. L. M. Lamers, L. J. Smith, \& A. Nota (San Francisco, CA: ASP), 201

Yusef-Zadeh, F., et al. 2006, ApJ, 644, 198

Zhang, J., et al. 2009, Phys. Rev. D, 80, 023007 